\title{
DEVELOPMENT OF TRAFFIC CONTROL FRAMEWORK FOR CONNECTED AND AUTOMATED VEHICLES USING OPTIMAL CONTROL THEORY
}

\author{
A Dissertation \\ Presented to \\ The Faculty of the School of Engineering and Applied Science \\ University of Virginia \\ In Partial Fulfillment \\ Of the Requirements for the Degree \\ Doctor of Philosophy \\ In Civil and Environmental Engineering \\ By \\ Seongah Hong
}

December 2017 


\section{ACKNOWLEDGEMENTS}

I thank God for leading me through all my studies until this moment.

I am indebted to a number of people without whom this research project would not have been possible. First of all, I express my sincere appreciation to my advisor, Prof. Byungkyu Brian Park, for providing me a platform to work on this project for my dissertation, for his incredible patience, rigorous challenges, insightful feedback and pertinent suggestions.

I also appreciate Prof. Andreas Malikopoulos at University of Delaware and Prof. Joyoung Lee at New Jersey Institute of Technology for their great contributions in mathematical modeling of the methodology and computer-based programming development which made this project even viable at all.

I would like to acknowledge the financial supports from the SMART Mobility Initiative of the Department of Energy (DOE), USA, and the Global Research Laboratory Program through the National Research Foundation of Korea (NRF) funded by the Ministry of Science, ICT \& Future Planning in South Korea.

Last but not least, I would like to deliver appreciation to my dad and mom for being my parents, my friends, and my inspiration. I am also grateful for my husband for the being so supportive and keeping me motivated, whom I love to be with forever in my life. 


\begin{abstract}
The state-of-the-art traffic operations strategies adhere to heuristic approach which uses numerical method to find approximate solutions that are close to the true solutions within a certain range. This is because of the complexity of representing traffic dynamics coupled with human drivers' behaviors. Obviously, such heuristic approach does not guarantee system optimality and is often not implemented in the field due to their computational burden and the need of calibration efforts entailed to the technique of the algorithm. Various traffic control algorithms have been consistently evolved in a way to improve computational efficiency to realize real-time operations.
\end{abstract}

Although there have been consistent improvements in effectiveness, the research to develop control strategy attaining true optimality is still lacking. Furthermore, it is still awaiting problem to optimize individual trajectory while considers vehicle platoon system.

This dissertation proposes an analytical approach-based control strategies using Pontryagin's Minimum Principle with an objective of minimization of control efforts (i.e., minimizing acceleration variations). The key merits of the proposed optimal control algorithm are: (i) it guarantees true optimal strategies; (ii) it is computationally less expensive; and (iii) it optimizes not only individual vehicle's longitudinal dynamics but also guarantees the optimality in terms of the vehicle platoon.

In particular, two problems are addressed in this dissertation: (i) optimal control on the speed of the automated vehicles before they enter a speed reduction zone on a freeway; and (ii) optimal control on the speed of the automated vehicles to follow the preceding vehicle. The control problem is formulated and solved using Hamiltonian analysis to provide an analytical, closed-form solution that can be implemented in real time. The 
solution yields the optimal acceleration/deceleration of each vehicle under the hard safety constraint of rear-end collision avoidance.

The developed algorithms are implemented and evaluated using the advanced microscopic simulation environment that is built in this research. A set of scenarios is tested to evaluate the performance in various aspects. The factors considered include traffic volumes and the market penetrations of automated vehicles. To evaluate the performance of the proposed algorithm, existing state-of-the-art algorithms that are comparable to the proposed algorithm are modeled and tested under the controlled conditions.

The optimal control algorithm shows significant improvements in mobility, fuel consumption, and traffic flow stabilization compared to those of the base case and the stateof-the-art algorithms under varying market penetrations of automated vehicles. For both of speed harmonization and the traffic flow stabilization control, the optimal control algorithm performs best under $100 \%$ market penetration of automated vehicles. The simulation results show that the travel time improves by $4-28 \%$ and the fuel consumptions improves by 6-21\% for different market penetrations of AVs. As for the traffic stabilization algorithm, the acceleration variations improve by up to $18 \%$ and the fuel consumptions improves by up to $30 \%$ compared to the base case of human driven vehicles for different market penetrations of AVs.

The experimental results in this dissertation demonstrate the feasibility of the control algorithm under mixed traffic of automated vehicles and human driven vehicles and provide quantitative assessment in various aspects of mobility, fuel economy and traffic flow stability compared to the existing practices. 


\section{TABLE OF CONTENTS}

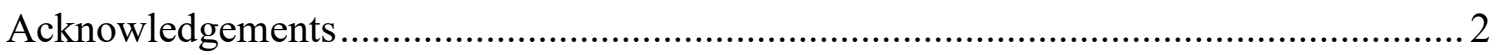

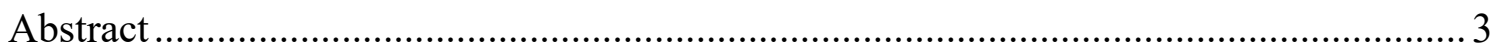

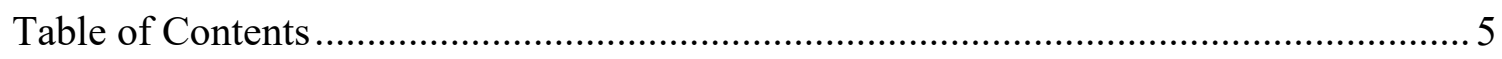

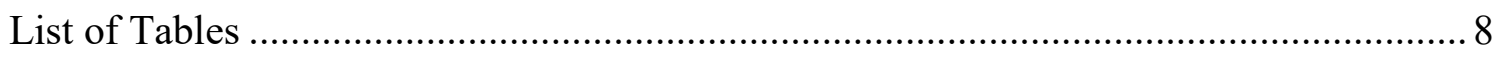

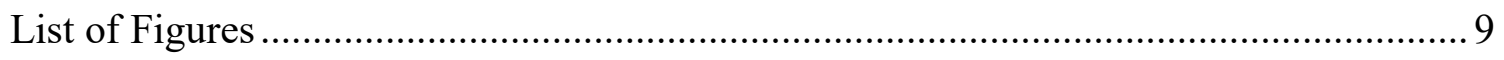

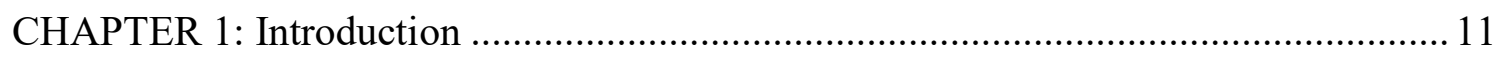

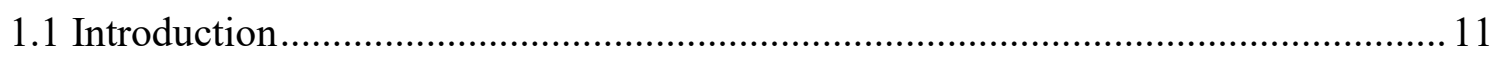

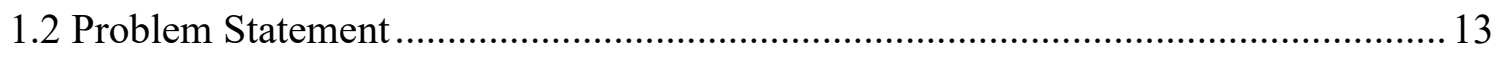

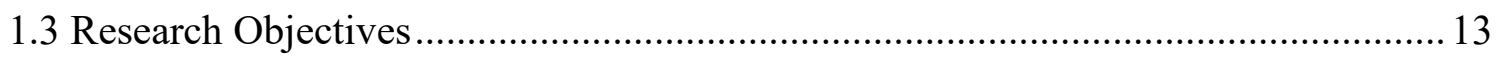

1.4 Dissertation Organization ............................................................................... 15

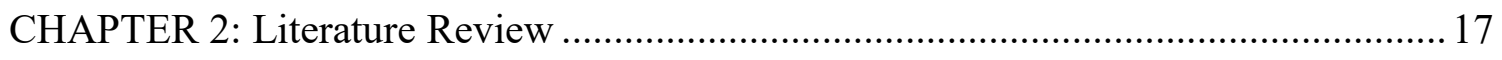

2.1 Advances in Traffic Control Strategies..................................................................... 17

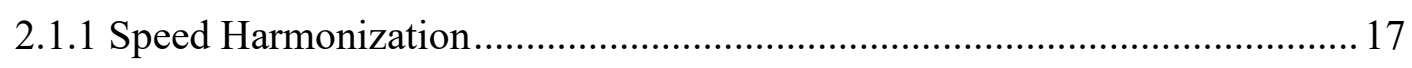

2.1.2 Traffic Flow Stabilization Control............................................................2 22

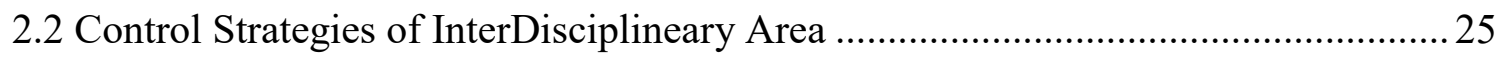

2.3 Challenges on the Mixed Traffic of Connected and Automated Vehicles with

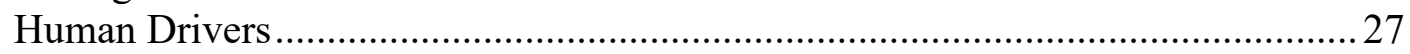

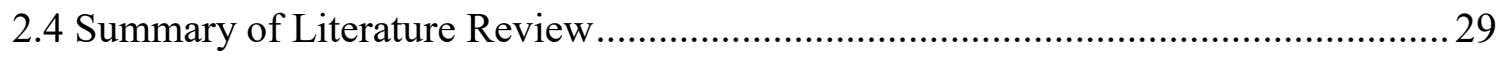

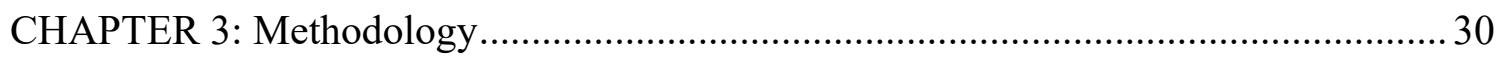

3.1 Development of Optimal Control Algorithm.......................................................... 30

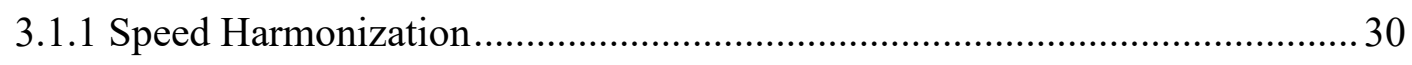

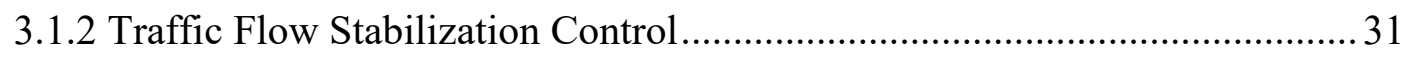

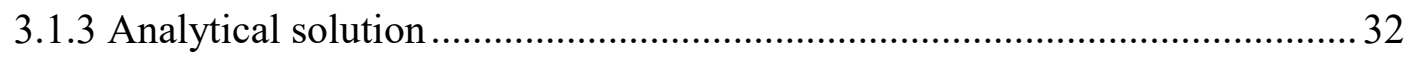

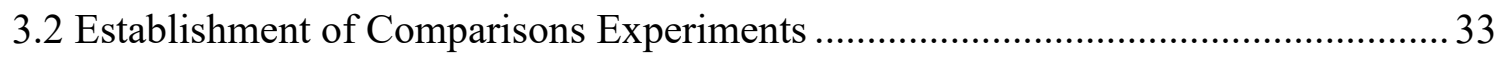

3.2.1 Base Case of Human Driven Vehicles........................................................... 33 


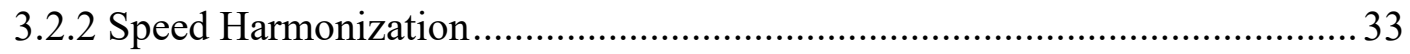

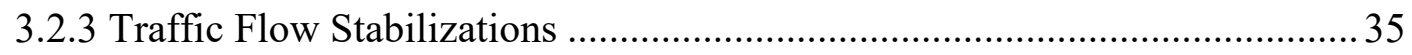

3.3 Development of Microscopic Simulation Platform .................................................... 35

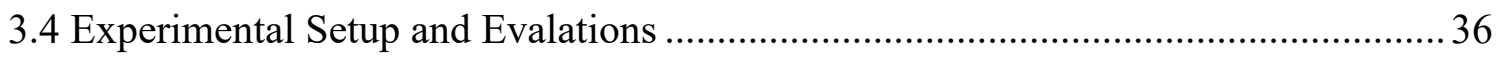

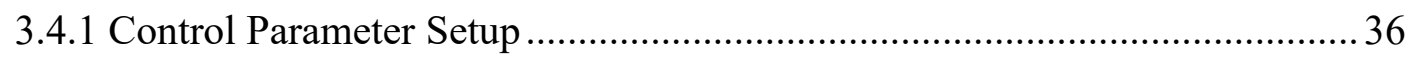

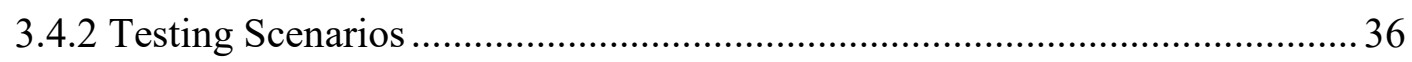

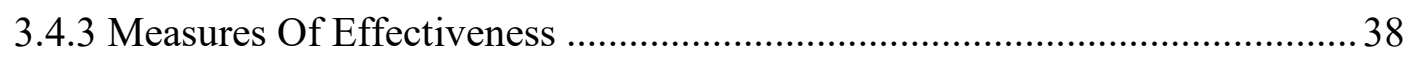

3.4.4 Statistical Testing and Minimum Sample Size ............................................... 40

CHAPTER 4: Development of Optimal Control Algorithm ............................................ 42

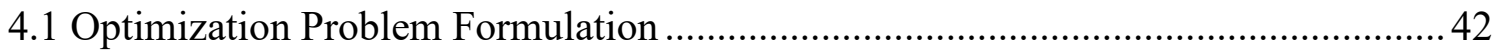

4.1.1 Speed Harmonization Algorithm ………………..................................... 42

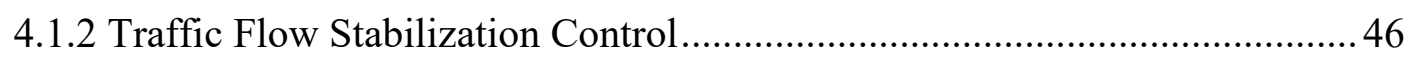

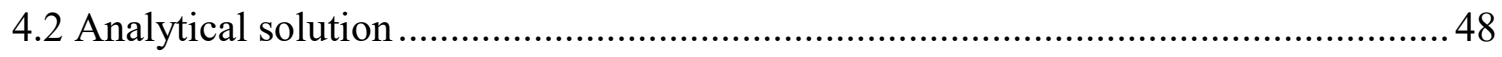

CHAPTER 5: Establishment of Comparison Experiments .............................................. 51

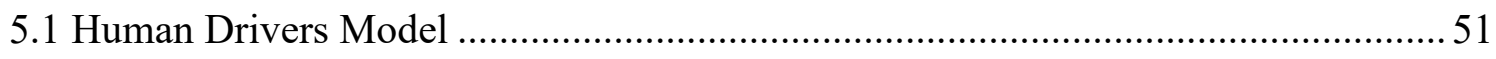

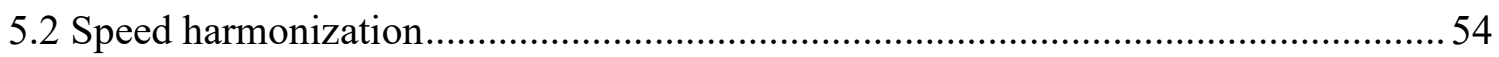

5.2.1 Speed Controlling Algorithm using Shock Wave Theory (SPECIALIST) ... 55

5.2.2 Simple Speed Harmonization ........................................................................ 56

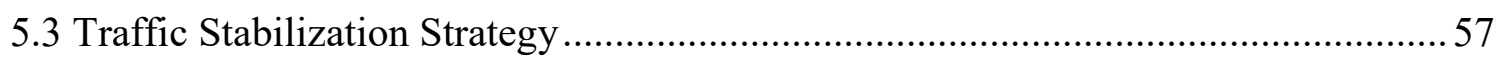

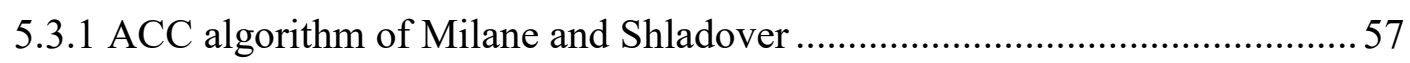

CHAPTER 6: Development of Microscopic Simulation Platform....................................59

6.1 Advanced Microscopic Traffic Simulation Tool .......................................................5 59

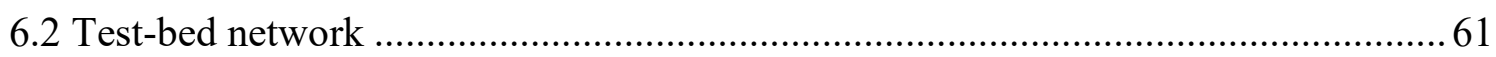

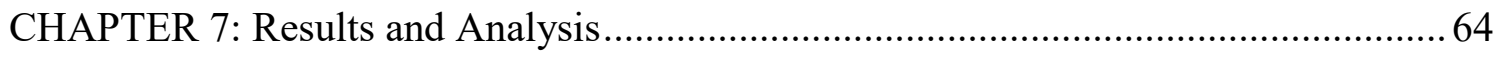

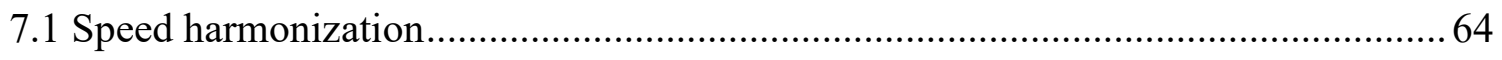

7.1.1 Under the perfect market penetrations of CAVs............................................... 64

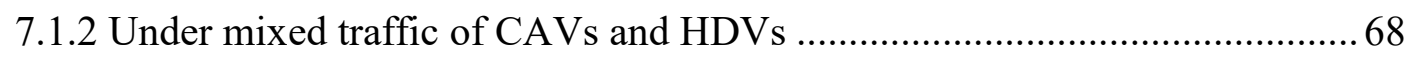




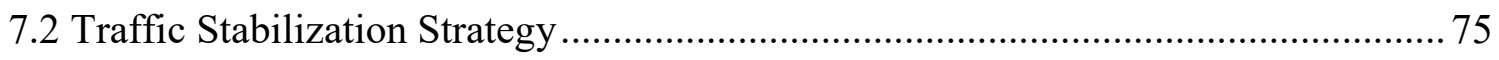

7.2.1 Under the perfect market penetration of AVs............................................. 76

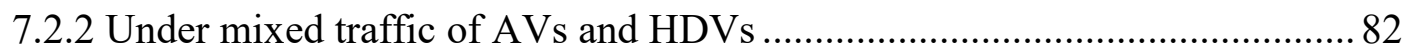

CHAPTER 8: Conclusions and Future Research.......................................................... 91

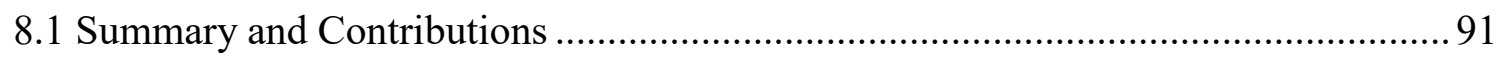

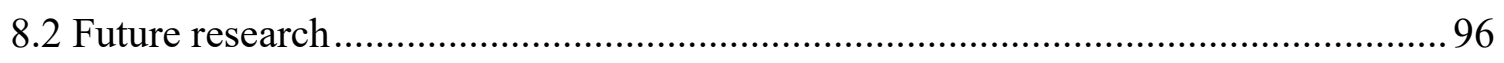

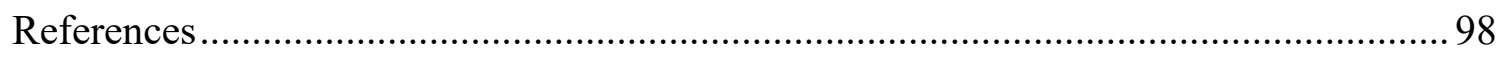




\section{LIST OF TABLES}

Table 1 Constraint Parameters of Optimal Control Algorithm ..................................... 36

Table 2 ANOVA Result for Travel Time ................................................................ 71

Table 3 ANOVA Result for Vehicle Throughput ................................................. 72

Table 4 ANOVA Results for Fuel Consumptions .................................................. 74

Table 5. Comparisons of MOEs between ACC and Optimal Control Algorithm for

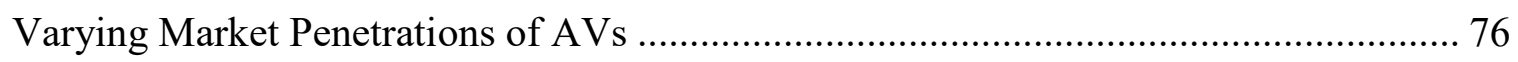

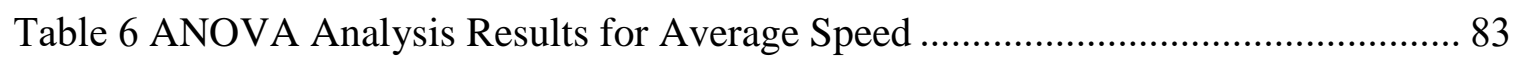

Table 7 ANOVA Analysis Results for Acceleration Variations ................................... 88

Table 8 ANOVA Analysis Results for Fuel Consumptions ...................................... 90 


\section{LIST OF FIGURES}

Figure 1 Overview of optimal control algorithm framework .................................... 32

Figure 2. Hierarchical sequence of the vehicle in the control zone............................. 43

Figure 3. Schematic presentation of optimal control algorithm for speed harmonization 45

Figure 4. Speed distribution of human driven vehicles for speed harmonization evaluation

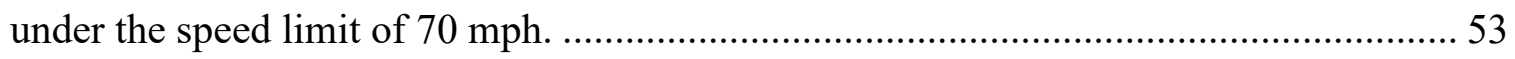

Figure 5. Speed distribution of human driven vehicles for traffic flow stabilization control evaluation under speed limit of (a) $30 \mathrm{mph}$ and (b) $45 \mathrm{mph}$, respectively. ..................... 54

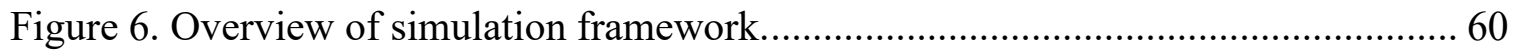

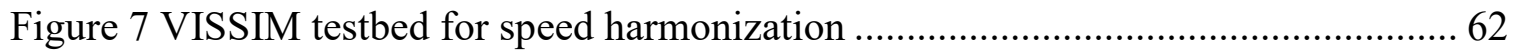

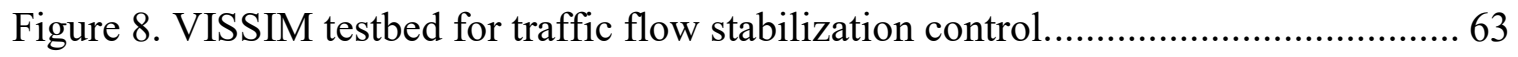

Figure 9. Performance of SPD-HARM algorithms under varying traffic volumes under

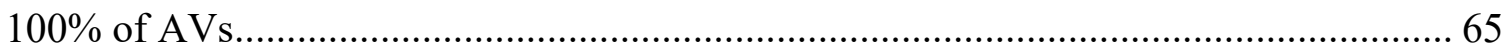

Figure 10. Travel time evaluations under varying penetrations of CAV ....................... 70

Figure 11. Vehicle throughput under varying penetrations of AVs.............................. 70

Figure 12. Fuel consumption under varying penetrations of CAVs ............................. 73

Figure 13. Speed trajectories of the case of $100 \%$ human driven vehicles..................... 78

Figure 14. Speed trajectories of the 100\% automated vehicles controlled by ACC

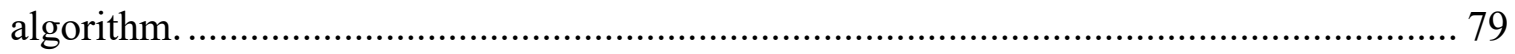

Figure 15 . Speed trajectories of the case of $100 \%$ automated vehicles controlled by

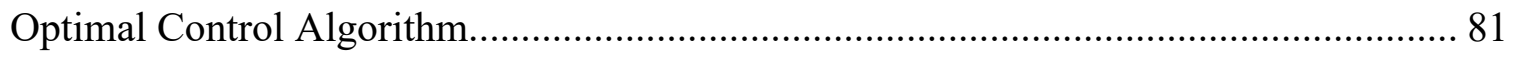

Figure 16. Average speed of ACC vs. Optimal Control Algorithm for varying market

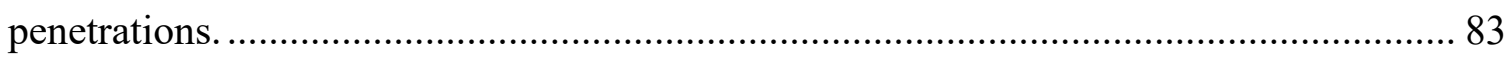


Figure 17. Per-vehicle acceleration variations of ACC vs. Optimal Control Algorithm for varying market penetrations of automated vehicles............................................. 85

Figure 18 Speed trajectory of ACC algorithm under $40 \%$ of AV . ............................. 86

Figure 19 Speed trajectory of the optimal control algorithm under $40 \%$ of AV............ 87

Figure 20. Fuel consumptions of ACC vs. Optimal Control Algorithm for varying market

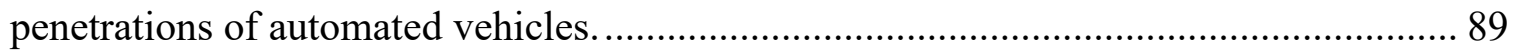




\section{CHAPTER 1: INTRODUCTION}

\subsection{INTRODUCTION}

Contemporary society suffers from traffic congestion. Adverse impacts from traffic congestion are ubiquitous in our daily lives: excess delay, reduced safety, increased fuel consumptions and environmental pollution, discomforts and annoyance during transportation activities. One distinct feature of the traffic flow system is that the throughput of the vehicles degenerates as the input exceeds the roadway capacity, instead of maintaining the vehicle throughput at the capacity level even in the absence of a bottleneck. According to Sugiyama et al. (2008), a traffic congestion is generated spontaneously only if the average vehicle density exceeds the critical value. Under this condition, the free flow state becomes unstable and even a minor fluctuation grows and the traffic state transits to congestion by the effect of collective behaviors. For this reason, a traffic control strategy needs to be deliberately designed in finding the control solutions using systematic approach in optimization, rather than heuristic approach.

In the US, one of the most widely practiced traffic operations applications is speed harmonization that has been used as a solution to address recurrent and non-recurrent traffic congestions and provide smoother and safer traffic flow along the freeway. With the advent of the technologies that allowed vehicles to be equipped with the high-resolution sensor devices, various traffic flow stabilization controls such as Adaptive Cruise Control (ACC) and Cooperative Adaptive Cruise Control (CACC) have been continuously improved as a key feature of the Advanced Driver Assistance System.

In the past several decades, the traffic operations strategies have been at its most prosperous stage qualitatively and quantitatively thanks to the leverage of advances in 
sensing and communication technologies as well as the collaborative movement of associating interdisciplinary approaches to deal with the traffic congestion problems.

In spite of the much of successes in improving the state-of-the-art strategies, the current practice of traffic operations still heavily rely on the numerical approach which limits the current control strategy to be reactive to the dynamically changing traffic conditions. The primary reason for pertaining to use the numerical heuristic approach is due to the challenges in modeling traffic flow behaviors that is largely probabilistic and dynamic.

However, with the emergence of connected and automated vehicles, some key features become possible: to collect real-time high-resolution traffic data using on-board sensors and communication devices; and to explicitly manipulate the vehicle's movement without the perception reaction time. Within this context, some transportation researchers and engineers proposed a new perspective to understand the traffic system from the perspective of multi-robot system and to solve for the optimality for the system through the interdisciplinary approaches. By leveraging the existing approaches in the systematic control area, the traffic control strategies can possibly benefit its robustness, transferability, and the efficiency of the algorithm while considering the restrictions on the physical context of vehicular dynamics, roadway infrastructures and operations system.

Even though we are facing the new era of how to define transportation and its every component (e.g., drivers, vehicles, infrastructure, and traffic operations system), the traffic control strategies still do not keep its pace with the advances in technology resources. Efforts to strive its bridge to the other disciplines such as cyber physical system should 
obtain further attention and the caliber of its state-of-the-art needs to be actively considered in the development of traffic control system.

\subsection{PROBLEM STATEMENT}

The traffic control strategies of various traffic applications have been experienced common paradigm shifts, which are: (i) fixed (or offline) scheme, (ii) reactive scheme, and (iii) proactive scheme. Such advancement has been factored by the advances in sensors and vehicle communication technologies which helped collecting detailed information on the traffic condition.

Through the advances in traffic control platform, the control strategy is consistently motivated to improve effectiveness in mobility and safety as well as the computational efficiency to realize real-time operations. However, it is the quite recent attempt to validate the true optimality of control algorithm in an analytical way. In addition, the efficiency of the control strategy is still not enough to implement to the field.

\subsection{RESEARCH OBJECTIVES}

To address the limitations of existing traffic control algorithms, this research proposes an analytical approach-based control strategies using Pontryagin's Minimum Principle (PMP) with an objective of acceleration minimization. The PMP deals with a dynamical system in the presence of constraints for the input variables. The PMP allows, from any point on an optimal state-space trajectory, the remaining trajectory to become optimal for the corresponding problem initiated at that point (Pontryagin 1987). In addition, unlike the Model Predictive Control (MPC) method, the optimal control theory approach 
does not require model development and calibration as the control scheme is subject to the vehicle dynamics of associated parameters when optimization is solved.

In summary, the contribution of the proposed algorithm to the existing literatures are:

- It optimizes acceleration variations while considering the minimum requirement of the mobility and safety;

- It guarantees true optimal strategies; and

- It is computationally less expensive.

The scope of this dissertation includes the application of the optimal control framework to the speed harmonization and the traffic flow stabilization control. The advanced microscopic simulation tool that integrates controller and simulator realized realtime implementation of the algorithm under various conditions and scenarios.

In this dissertation, the major contribution is to establish a platform where new frame of traffic control strategy is developed by adapting smart control strategy in other disciplines such as robotic control system in consideration of the restrictions of traffic system. In particular, an analytical approach widely utilized in the system control area is reconstructed to apply to address the traffic control problems in various traffic application cases such as speed harmonization and traffic flow stabilization control. 


\subsection{DISSERTATION ORGANIZATION}

The remaining part of this dissertation is organized as follows:

Chapter 2 summarizes the literature reviews. This chapter reviews the advances of the traffic control strategies at the perspectives of the nature of the strategy such as the reactive approach and the prospective approach. Traffic operations applications of speed harmonization and the traffic flow stabilization control are included.

Chapter 3 provides an overview of the methodology used in this research. This includes a discussion of the development of the optimal control algorithm framework, establishment of the advanced simulation platform, and the experimental designs for evaluations.

Chapter 4 describes the optimal control algorithm that are developed in this research. Descriptions of problem formulations, process to derive analytical solutions as well as the application to the traffic operations applications of speed harmonization and the traffic flow stabilization control are presented.

Chapter 5 explains the establishment of comparison experiment designs that are modeled to assess the proposed optimal control algorithm in this research.

Chapter 6 describes the advanced microscopic simulation tool that is integrated to realize the on-time implementation of the proposed algorithm and to assess the feasibility and the performance under various scenarios. VISSIM, one of the most widely used 
microscopic traffic simulation software is utilized upon the COM interface platform. Data extraction and evaluation process embedded to the simulation tool is explained.

Chapter 7 examines the results of the testing on the hypothetical networks. These tests assessed the impact of the various factor combinations on the mobility and fuel consumptions. The factors of considerations include traffic volumes and market penetrations of automated vehicles. This results are used to explore the feasibility of the algorithm and/or the sensitivity of the performance in various aspects, which helps identify the factors of considerations when it comes to the practical implementation in the future.

Chapter 8 provides overall summary and conclusions for the research. Contributions and challenges of the research are presented, which is followed by the future research items. 


\section{CHAPTER 2: LITERATURE REVIEW}

\subsection{ADVANCES IN TRAFFIC CONTROL STRATEGIES}

\subsubsection{Speed Harmonization}

Traditionally, the SPD-HARM has been realized through variable message signs (VMS), variable speed limit (VSL) and the rolling SPD-HARM (a.k.a., pace-car technique) (Roberts 2012). Both VMS and VSL systems employ the display gantries mounted along roadways to deliver messages or control schemes. Another method for achieving SPDHARM is the rolling SPD-HARM, which uses designated patrol vehicles entering the traffic to hold a traffic stream at a lower speed, and thus, traverse the congestion area smoothly while mitigating shock waves.

The performance of SPD-HARM varies depending on the control approach, characteristics of the topology, and driving behaviors. Even though the benefits in travel time through SPD-HARM has been debatable especially when dealing with the peak hour traffic (Kwon et al. 2011; Roberts 2012), it is widely accepted that SPD-HARM increases vehicle throughput at the bottleneck. It has been shown that the vehicle throughput can be increased by 4-5\% via VSL system and by 5-10 \% via rolling SPD-HARM implemented in European countries with significant benefits in safety since personal injury crashes reduced about 30-35\% (Juan et al. 2004). The environmental impacts of SPD-HARM were also substantial demonstrating reduction in vehicle emissions by $4-10 \%$ (depending on the pollutants), and fuel consumption by 4\% (Juan et al. 2004). 
The application of SPD-HARM has been mainly evolved through VSL which appeared to be more effective and efficient than VMS and the rolling SPD-HARM (Roberts 2012; Robinson 2000). Most of the current practice of VSL systems employ the proactive approach in which they apply a control action beforehand and then anticipate the behavior of the system (Khondaker and Kattan 2015). Even though this proactive approach has made VSL as a popular method over the years, it is likely to find a sub-optimal solution since it is based on a heuristic approach (Frejo and Camacho 2012).

The SPD-HARM strategies can be categorized into reactive approach and proactive approach. The reactive approach initiates the operation at a call upon a queue detected, and it uses immediate traffic condition information to determine the control strategy for the subsequent time interval. While the reactive approach allows to remedy the bottleneck with real-time feedback operations, it has limitations related to time lag between the occurrence of congestion and applied control (Papageorgiou et al. 2008).

In contrast, the proactive approach has the capability of acting proactively, while anticipating the behavior of traffic flow (Khondaker and Kattan 2015). Thus, it can predict bottleneck formations before they even occur, while potential shock waves can be resolved by restricting traffic inflow. In addition, the nature of predictions of proactive VSL methods allows for a systematic approach for network-wide coordination which supports system optimization, whereas reactive approach is restrained to a localized control logic. The remaining part reviews on the reactive and the proactive control approaches at greater detail. 


\section{Reactive Speed Harmonization}

The first field implementation of SPD-HARM was the VSL system in the German motorway A8 corridor in Munich in 1965 (Schick, 2003). During the early 1960s, the US first implemented SPD-HARM using VMS on a portion of the New Jersey Turnpike (Robinson 2000). These SPD-HARM systems required human interventions to determine the messages or speed limits based on the conditions such as weather, traffic congestion and construction schedules. Since 1970s, advances in sensor technologies and traffic control systems allowed the SPD-HARM to automatically operate based on the traffic flow or weather conditions using various types of detectors. The earlier VMS and VSL implementations were often at the purpose of addressing safety issues under work zone areas or inclement weather conditions through the qualitative assessment on the conditions (Robinson 2000).

In 2007, SPD-HARM started focusing on improving traffic flow mobility by the use of quantitative information. The VSL systems implemented in the M42 motorway at Birmingham, UK, and Washington State Department of Transportation (Brinckerhoff et al. 2008) used algorithms which are automatically activated based on pre-defined threshold of flow and speed measured by detectors embedded in the pavement. The systems display the lowered speed limit within a "control zone" of a pre-defined length.

Development of VSL algorithms using quantitative approaches were more active in academic area: various reactive VSL algorithms were developed and evaluated under a diversity of scenarios. Park \& Yadlapati (2003) and Chen \& Ahn (2015) developed VSL algorithms for work zone areas by using safety surrogates and speed information to improve safety and mobility. They successfully showed that their VSL system 
outperformed the existing VSL algorithms, especially with the traffic demand fluctuations (Park and Yadlapati 2003).

The VSL algorithm developed by Juan et al. (2004) was tested under a comprehensive set of testing scenarios with varying traffic volumes. The study found that the performance of VSL is a function of the traffic volume levels, thus the benefit from the VSL can be most achievable by integrating the ramp metering control adjacent to the VSL system (Juan et al. 2004).

One of the most recognized VSL algorithm that used reactive approach was the VSL system implemented in the I-35W within the Twin Cities Metropolitan area (Kwon et al. 2011). The algorithm identifies the moving jam based on the deceleration rate between adjacent spots. The field evaluation showed the reduction in average maximum deceleration by $20 \%$ over the state-of-the-art in that area while improved the vehicle throughput at the bottleneck areas.

The group of reactive SPD-HARM algorithms has consistently showed improvements in many aspects such as reliability, safety and environmental sustainability by providing adequate feedback to the dynamic traffic conditions. However, the capability of reactive control is limited as it can be only effective after a bottleneck occurs while it mainly depends on heuristics. 


\section{Proactive Speed Harmonization}

The necessity of a systematic approach for preventing adverse impacts from impending shock waves eventually led to the development of the proactive SPD-HARM system. The proactive VSL approach was first proposed by Alessandri et al. (1998) adopting Kalman Filter aimed at estimating impending traffic status based on the timeseries traffic measurements (Welch and Bishop 2006). Given with the estimated traffic flow, the proactive VSL approach derives a control policy that minimizes various cost functions (e.g., average travel time, summation of square densities of all sections). Although this effort initiated prediction-based VSL systems, the prediction using a timeseries approach was not robust, especially under unexpected traffic flow disturbances, since it heavily relied on the empirical patterns.

A pioneering effort in developing a proactive VSL system was made by (Hegyi et al. 2003) using model predictive control (MPC). The key aspect of that work is that it prevents traffic breakdown by decreasing the density of approaching traffic rather than focusing on reducing the speed variances. Using MPC, which enabled a network-wide optimization, a series of VSL systems can be coordinated for system-wide optimization that eventually aims at preventing upstream delays.

Another MPC-based proactive VSL system was proposed in ( $\mathrm{Lu}$ et al. 2010) focusing on creating a discharge section immediate upstream of the bottleneck to regulate traffic flow into the bottleneck that remains close to its capacity. With the intention to influence the motorway mainstream, a traffic flow control approach was proposed in (Carlson et al. 2010), which adapted a discrete-time dynamic control method using a 
suitable feasible-direction algorithm (Papageorgiou and Marinaki 1995), that can yield feedback control policies.

These approaches have showed substantial improvements in vehicle throughput, safety, equity, and driver acceptance through microscopic simulation studies (Carlson et al. 2010; Hegyi et al. 2003, 2005a; b). However, there are significant challenges in practical applications associated with computational requirements.

\subsubsection{Traffic Flow Stabilization Control}

Sugiyama et al. (2008) has brought up a new analysis on the traffic congestion phenomenon: traffic bottleneck is only a trigger for the congestion and not the essential origin of it. In their study, they provided the first experimental verification that a traffic congestion can be generated in the absence of a bottleneck, through the test in the real motorway ring-road and 22 trained drivers. They found that, in any case, the instability of the free flow caused by exceeding the critical density leads to the transition to a congestion state by the collective effect of vehicles such as the enhancement of fluctuations or some perturbation (Sugiyama et al. 2008).

Stern et al. (2017) revisited the problem of traffic waves even under free flow state when the vehicular density exceeds a critical threshold. They proposed an algorithm to dampen the speed oscillations using one vehicle. They developed control strategy called Proportional-Integral (PI) controller with saturation, which is a modified version of the PI controller - the widely used controller in industrial applications. The basic idea on the modified PI control is that control action is saturated at small gaps to avoid collisions, and long gaps to avoid slowing down of traffic (Stern et al. 2017). Even though they 
successfully showed substantial reductions in speed standard deviation, excessive braking, and fuel consumptions, the approach associates reactive method and does not guarantee optimal state for the system.

Meanwhile, as a key feature of the Advanced Driver Assistance System (ADAS), the Adaptive Cruise Control (ACC) is one of the fastest-growing control system with steadily increasing rates of adoption of industry-wide quality standards (Hale and Lu 2016). More advanced of ACC is cooperative systems, so called Cooperative Adaptive Cruise Control (CACC), where the equipped vehicles are connected with each other. ACC and CACC system rely on the input from multiple data sources such as LiDAR, radar, image processing, and additional inputs for $\mathrm{CACC}$ that are obtained by connecting to the wireless network, such as Vehicle-to-Vehicle (V2V), or Vehicle-to-Infrastructure (V2I) systems.

One of the most recognized models to describe ACC vehicle behavior is Intelligent Driver Model (IDM). IDM has a driving strategy that varies parameters according to traffic situations to mitigate congestion at bottlenecks (Kesting et al. 2007). Their results showed significant improvement in string stability for the vehicle platoon formation and provided favorable traffic flow results at even low AV market penetrations, compared with the previously presented algorithms in the literatures (Seiler et al. 2004; Shaw and Hedrick 2007).

The most extensively studied ACC model is a linear feedback controller, where the vehicle acceleration is proportional to the distance gap and the derivative of the gap at carfollowing conditions (Zheng et al. 2016). The resultant car-following behavior of this controller is that ACC vehicles maintain a constant time gap at equilibrium condition. Such 
car-following behavior control strategies influence the individual vehicle behavior, and thus induce changes in aggregate traffic flow behaviors to be more stable.

In a while, Cooperative Adaptive Cruise Control (CACC) technologies allow a vehicle to automatically adjust its speed to maintain a safety distance from the preceding vehicle and the very leading vehicle of the platoon, based on information gathered from stationary or mobile devices. Milanes and Shladover (2014) developed ACC and CACC algorithms - the sophisticated variant system of their ACC algorithm by integrating an accurate models of the dynamic responses of the controller to support practical implementation of the system to the field. The details on the algorithm are discussed in Chapter 5.3. It is worth noting that the ACC and CACC algorithms developed by Milanes and Shladover (2014) are designed with consideration of realistic predictions, unlike many other ACC systems, and is actually more stable than some other existing ACC and CACC systems such as IDM and the existing commercial ACC algorithms (Milanés and Shladover 2014). However, even its benefits on the stability and accuracy, the ACC and CACC system are not able to overcome the challenges associated with the control policy that guarantees system optimality. 


\subsection{CONTROL STRATEGIES OF INTERDISCIPLINEARY AREA}

The solution to the problem of traffic control strategies has been sought for in the area of robotics. The traffic system, especially the traffic stream of automated vehicles, can be considered as the multi-robot system with the constraints of physical features of roadways.

Above all approaches, extensive research has gone into developing different methods of coordinating multiple nonlinear dynamical agents based on solving consensus problems (J. Qin et al. 2012; Wang et al. 2014). One of the most recent studies was done by Quintero et al. (2013) who developed a control strategy upon a leader-follower topology, where the problem of determining the follower's policy was setup as a stochastic optimal control problem using dynamic programming. In a while, researchers obtained impressions from how biological and artificial animals behave to develop control strategies of robots. Especially the flying insects or birds motivated to model flocking behaviors which basically emerge from a group when individual moves according to their own neighbors' behaviors, while adhering to rules for separation, alignment, and cohesion (Liu and Passino 2000). Various algorithms were used to obtain solutions to the behavioral based approach and mostly evolutionary computation algorithms such as Artificial Bee Colony (ABC) algorithm, and global-best-guided Artificial Bee Colony (GABC) algorithms were used under decentralized system (Golestan et al. 2016; Jovanovi et al. 2017; Rajasekhar et al. 2016). Another notable approach was the Reinforcement Learning (RL) approach. Hung et al. (2017) developed a flocking model which did not require models and realize on-line learning through RL approach. Such model-free RL framework 
enabled the model to be applied to different platforms easily, which imposes greater adaptability to changing environments (Hung et al. 2017).

In general, it was successful to comprehend the traffic system as multi-robot system and to prove improvements in the system performance. However, all of those algorithms requires time to achieve the solution, which may not be suitable for the on-line applications of traffic control and operations. More recently, the challenges upon the computational complexities and the real-time applications of the traffic control strategies have been attempted to be solved through the remote computing framework. The concept of vehicular cloud - a remote computing framework specific for transportation system, has been proposed to mitigate the computational efforts by transferring the computational load to the computers in the cloud using the capability of mobile system (Khayyam et al., 2012). Ozatay et al. (2012) developed a system using the vehicular cloud, where each vehicle uploads its information such as speed and location to the cloud, and the cloud then derives the optimal control strategy for a vehicle. It was notable contribution to invent the framework of remoting computing system, but the computational efficiency was still limited when extra delay was loaded with traffic congestion. Qiu et al. (2016) improved the framework of Ozatay et al. (2012) by considering the driving features of close vehicles on a road (e.g., similar speed and gap distances), they proposed to group multiple vehicles within a certain range and let the leader vehicle in each group to upload the group information to the cloud and the controller drives the control strategy of each vehicle in the group. They proved the feasibility of the real-time applications through the real-world experiments using simulation tools, but they are limited to validate the safety issues under real applications. 


\subsection{CHALLENGES ON THE MIXED TRAFFIC OF CONNECTED AND AUTOMATED VEHICLES WITH HUMAN DRIVERS}

A pool of literatures demonstrates benefits that AV or CAV can bring to our society. The real-time high resolution vehicle trajectory data that is provided to the AV or CAV can be essentially used to better understand the current and upcoming traffic conditions, and become a basis for the real-time traffic control. Many studies in this area found that a small market penetration percentage of $\mathrm{AV}$ or $\mathrm{CAV}$ can yield significant benefits.

Meanwhile, although the technologies of AV and CAV may soon be commercialized, highway traffic is unlikely to exclude human divers in the foreseeable future. According to Milakis et al. (2016), the fully automated vehicles are expected to be commercially available between 2025 and 2045. Market penetration rates are expected to be between $1 \%$ and $11 \%$ in 2030 , and it will increase to be between $7 \%$ and $61 \%$ in 2050 (Milakis et al. 2016). Subject to such limitations of viability at this stage, in a traffic stream containing mixed traffic of AVs and human drivers, human factors will continue to affect vehicle motions and overall traffic performance. Thus it is called for an evaluation considering the partially $\mathrm{AV}$ penetrations mixed with human drivers.

There have been several studies (Van Arem et al. 2006; Cheng et al. 2013; Olia et al. 2015; Rajamani and Zhu 2002) which investigated the impact of the automated vehicle market penetrations on the highway operational measures and concluded that the market penetrations significantly improved the capacity of a roadway or vehicle throughputs.

Recent studies related to the development of AV applications focused on the impact analysis for different market penetrations. Lee et al. (2013b) developed an adaptive 
intersection control algorithm called Cumulative Travel-time Responsive (CTR) equipped with a stochastic state estimation technique to enable the implementation under mixed traffic of AVs and human driven vehicles. They found that at least $30 \%$ of automated vehicle market penetration should be reached to realize the benefits of the algorithm over the base case of optimized actuated control. Park and Lee (2009) developed and evaluated the advanced route-guidance system under different market penetrations of automated vehicles. They found that the travel time, fuel consumption and emissions were all maximized at the market penetration of $75 \%$, not the $100 \%$ market penetration. It was due to the extra delay that was occurred because of having more vehicles that used the same routes following the system guidance.

However, most existing studies on the mixed traffic of AV and CAV make simple assumptions of human behavior. The traffic congestion is closely related to complex and non-deterministic human driving behavior. The next stage of a research should investigate proper consideration of human driven vehicle behaviors based on the psyco-physical modeling and explore the strategies for AVs to reconcile with the human drivers in terms of the safety violations. 


\subsection{SUMMARY OF LITERATURE REVIEW}

The key lesson learned from the literature review is that the state-of-the-art traffic control strategies of speed harmonization and traffic flow stabilization control needs better control approach that can address major challenges:

- Consideration in system optimality

- Computations efficiency; and

- Proper strategy to deal with human driven vehicle in the mixed traffic.

To address those problems, the optimal control algorithm framework is developed and evaluated in this dissertation. The control problem is formulated to provide an analytical, closed-form, optimal solution that can be implemented in real time. The solution yields the optimal acceleration/deceleration of each vehicle in the upstream, and thus it controls the time that each vehicles in the control zone. Furthermore, the control strategy provides the conditions under which the rear-end collision avoidance constraint does not become active at any time during the control, so that the AVs or CAV can reconcile with human driven vehicles under the mixed traffic. 


\section{CHAPTER 3: METHODOLOGY}

\subsection{DEVELOPMENT OF OPTIMAL CONTROL ALGORITHM}

The first task is to develop an optimal control algorithm framework and provide analytical solution using Pontryagin's Minimal Principal (PMP). Using PMP method, the closed-form solution for the problem is formulated. The control policy is treated as a sequential minimization problem for each vehicle starting from the leading vehicle. The dynamics of the vehicle is considered in the problem. It is important to emphasize that this analytical solution allows the implementation of online optimal control that updates its optimality every control interval, which supports a dynamically changing traffic flow as well as the human driven vehicles effectively. Problem for each traffic control strategy is formulated as in the followings.

\subsubsection{Speed Harmonization}

To formulate a control problem for the context of speed harmonization, a scenario of speed reduction zone of $35 \mathrm{mph}$ followed by the roadway with the speed limit of 70 mph. The control is implemented within the fixed length which is located immediate upstream the speed reduction zone.

The optimal control algorithm for the speed harmonization is formulated to aim at deriving the optimal acceleration profile when the leading vehicle accelerates or brakes between the current time and the time to enter the bottleneck location (i.e., speed reduction zone in this case). Within this time horizon, the constraints on the speed and the location of a vehicle is estimated accordingly: the final location is fixed as the entering point of the

speed reduction zone and the final speed is the speed limit of the speed reduction zone. At 
the same time, however, the time that the subject vehicle enters the speed reduction zone is over-ruled by the imposing rear-end collision constraint of preceding vehicles. In addition, under the low traffic volume cases, the final constraint of the vehicle entering does not need to catch up on the preceding vehicle to maintain safety minimum distance. Such various conditions are jointly considered in formulating the control algorithm so that the solution represents the effective traffic control with adequate behavior patterns.

\subsubsection{Traffic Flow Stabilization Control}

The traffic flow stabilization control is formulated to mitigate the traffic speed fluctuations that are even occurring under simple car-following situation without external bottleneck by the use of the optimal control algorithm framework.

The basic idea of the traffic flow stabilization control is to make a vehicle maintain a desired time headway from the preceding vehicle unless the trajectory violates the safety. First, the control strategy identifies if the current condition signifies potential safety violations based on the speed and location information of the subject vehicle and the preceding vehicle. If it is signified as safe to drive at the optimal speed, the vehicle is likely to smoothly converge to the optimal speed. If it is signified as unsafe to do so due to the preceding vehicle, then the algorithm provides the optimal strategy that is constrained by the preceding vehicle's information. Different from the speed harmonization algorithm, the traffic flow stabilization control aims at maintaining the same time headway time that is predefined by users. 


\subsubsection{Analytical solution}

The Pontryagin's Minimum Principle (PMP) is applied to find the analytical closed-form solution for the problem forumlated under the respective constraints of the SPD-HARM and traffic flow stabilization control. The problem is treated as a sequential minimization problem for each vehicle $i$. The overall process of the framework of the optimal control algorithm is illustrated in Figure 1.

\begin{tabular}{|c|c|}
\hline \multicolumn{2}{|c|}{$\begin{array}{l}\text { Define Hierarchical Vehicle Sequence within } \\
\text { the Control Zone based on the First-In-First-Served } \\
\text { indexed by } i=1,2, \ldots, \mathrm{n}\left(\text { i.e., } \mathrm{x}_{1}>\mathrm{x}_{2}>\ldots>\mathrm{x}_{\mathrm{n}}\right)\end{array}$} \\
\hline \multicolumn{2}{|c|}{ Define Initial and Final Conditions for Each Vehicles } \\
\hline $\begin{array}{l}\text { Speed Harmonization } \\
\text {-Define the initial and final locations } \\
\text { and speeds for vehicle } \mathrm{i} \\
\qquad \begin{array}{l}x_{i}\left(t_{i}^{f}\right)=x_{i-1}\left(t_{i-1}^{f}\right)+\delta \\
v_{i}\left(t_{i}^{f}\right)=v_{i-1}\end{array}\end{array}$ & 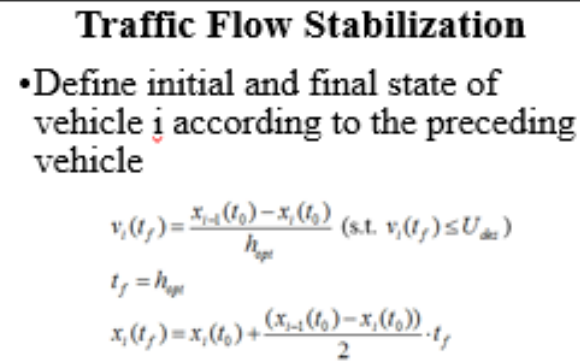 \\
\hline \multicolumn{2}{|c|}{ Solve for Analytical Solution of the Optimal Control Problem } \\
\hline \multicolumn{2}{|c|}{$\begin{array}{l}\min _{u_{i}} J=\min \frac{1}{u_{i}} \frac{1}{2} \sum_{i=1}^{n} \int_{\ell}^{t} u_{i}^{2} d t \\
\text { where } \\
i=\text { vehicle index } \\
n=\text { the number of vehicles in the control zone } \\
u_{i}=\text { acceleration of a vehicle } i \text { (control input) }\end{array}$} \\
\hline subject to vehicle dynamics & $\mathrm{d}$ initial and final conditions. \\
\hline
\end{tabular}

Figure 1 Overview of optimal control algorithm framework 


\subsection{ESTABLISHMENT OF COMPARISONS EXPERIMENTS}

To evaluate the effectiveness of the proposed optimal control algorithm, various comparisons groups are selected and developed under the same control setting. In this research, the comparisons experiment considered (i) baseline of no-control and (ii) stateof-the-art algorithm(s) for each traffic control strategy.

\subsubsection{Base Case of Human Driven Vehicles}

The baseline of no-control associates with human drivers based on the Wiedemann 99 psycho-physical car-following model that is widely accepted as a good representation of perceptive behaviors of human drivers under uninterrupted traffic flow.

To begin with, the VISSIM model is carefully calibrated by referring to the guideline of the Highway Capacity Manual (HCM) 2010 manual (TRB 2010) to represent the typical two-lane highway conditions. In this dissertation, the calibrations process involves adjusting the standstill distance between two vehicles and the headway time which a driver wants to maintain. To account for differences in the driving behavior of human drivers, the desired speed distribution is closely setup as normal distribution with the average of speed limit and the range of $\pm 3 \mathrm{mph}$ of the speed limit.

\subsubsection{Speed Harmonization}

In SPD-HARM strategy evaluations, two different types of SPD-HARM strategies are considered for the comparisons target: one is of the Variable Speed Limit system conventional technique of SPD-HARM, and the other is of the individual vehicle-based 
SPD-HARM which premises that the controlled vehicles are equipped with the suitable sensor technique.

Speed Controlling Algorithm using Shock Wave Theory (SPECIALIST)

The SPECIALIST is a proactive VSL algorithm that projects traffic conditions in the near future using the Model Predictive Control (MPC) method. This algorithm is chosen it is considered as most advanced - less computations required, only including a few parameters with physical interpretations that makes field implementations viable theoretically.

The basic idea of the SPECIALIST is that the variable speed limit on the gantry is determined based on the logic that determines the implementation duration and length of control using the information on the different traffic states along the freeway.

For the comparisons evaluations, SPECIALIST is modeled using C\# programming and implemented in the VISSIM using its COM interface. Since SPECIALIST is based on a mesoscopic model that utilizes the spot-based measurement collected at a fixed location and aggregated for a certain period of time, detector stations are evenly embedded at every 250 feet along the corridor to estimate the local traffic states.

\section{Simple Speed Harmonization}

For a fair comparison, a vehicle-based speed harmonization strategy is considered in comparisons evaluation. The Simple Speed Harmonization (SH) Algorithm (Ma et al. 2016) that is developed through the support of US DOT is chosen with its successful validation of field implementation (Ma et al. 2016) and the effectiveness in mitigating the traffic oscillations has been updated by Learn (2016). It has to be mentioned that the Simple 
SH is the simplified version of the earlier work of SPD-HARM algorithm (Lee et al. 2013a) that is modified to fit into the field implementation of controlling individual CAVs. The Simple SH basically uses the proportions of the relative speed and the location of the preceding vehicles to determine the control speed of the following vehicle.

\subsubsection{Traffic Flow Stabilizations}

\section{ACC algorithm of Milanes and Shladover}

In traffic flow stabilization control evaluations, the ACC algorithm (Milanés and Shladover 2014) is selected to compare with the proposed optimal control strategy that does not require communications between vehicles. This ACC algorithm is considered the most advanced with the validation with the real experimental data using CAVs. The algorithm basically provides the smooth speed trajectory which minimizes the acceleration value. The objective function is formulated as a function of the relative distance and the speed differences between the subject vehicle and the preceding vehicle.

\subsection{DEVELOPMENT OF MICROSCOPIC SIMULATION PLATFORM}

A simulation framework is established by integrating a controller and a simulator using the Visual C\# programming environment to evaluate the effectiveness of the proposed optimal control algorithm. The optimal control algorithm is coded using MATLAB language Dynamic Link Library (DLL) interface programming to allow data exchange with other external programs within the framework. A simulation test-bed network is developed under VISSIM, and it is integrated into the framework by using its COM interface. 


\subsection{EXPERIMENTAL SETUP AND EVALATIONS}

\subsubsection{Control Parameter Setup}

The control parameters related to the constraints of the optimal control algorithm are summarized in Table 1. The maximum/minimum speed and acceleration values are determined considering vehicle technical feasibility of automated vehicles (Gouy et al. 2013) and the suggestions of the guideline published by the Federal Highway Administration (Dowling et al. 2004). To ensure constancy between the controller and the traffic simulator, the optimal control strategy is calculated and updated every 0.1 seconds which is identical with the VISSIM microscopic simulator resolution.

\section{Table 1 Constraint Parameters of Optimal Control Algorithm}

\begin{tabular}{|l|c|}
\hline \multicolumn{1}{|c|}{ Parameter } & Value \\
\hline Minimum speed & $20 \mathrm{mph}$ \\
\hline Maximum speed & $\begin{array}{c}\text { Respective speed limit } \\
\text { on the roadway }\end{array}$ \\
\hline Maximum acceleration & $15 \mathrm{feet} / \mathrm{s}^{2}$ \\
\hline Maximum deceleration & -15 feet $/ \mathrm{s}^{2}$ \\
\hline Minimum gap distance & 20 feet \\
\hline
\end{tabular}

\subsubsection{Testing Scenarios}

To design testing scenarios, two major factors are considered to give impact on the performance of the control algorithm: traffic volumes and the market penetrations of automated vehicles. 
For evaluating the SPD-HARM algorithms, three traffic volume cases are considered as: (i) traffic volume of $10 \%$ less than the capacity, (ii) traffic volume at the capacity, and (iii) traffic volume of $10 \%$ greater than the capacity. On the contrary, in evaluation of the traffic flow stabilization control, single case of traffic volume is tested since the problem is the matter of generating individual car-following strategy, thus changes in traffic volume would not make difference in the results.

To reflect the impact of level of market penetrations of automated vehicles, the testing scenarios considered the range of 0 to $100 \%$ at $10 \%$ increments of automated vehicle penetrations. For each case of imperfect market penetrations of automated vehicles, the automated vehicles are randomly distributed.

For the traffic flow stabilization control, the case of $5 \%$ of automated vehicles are additionally tested in which the case includes three automated vehicles which forms a row across the three-lane roadway unlike the other cases of imperfect market penetrations where the automated vehicles are randomly distributed among the human driven vehicles. This specific case of 5\% market penetration is designed to explore the impacts of having a "front" of automated vehicles which serves as a pace-car which are following different traffic flow stabilization controls. In this study, the location of the row of three automated vehicles are varied: one case where the three vehicles are located in the $2^{\text {nd }}$ row, and the other case of the three vehicles located at the $10^{\text {th }}$ row, or at the center of the traffic platoon. 


\subsubsection{Measures of Effectiveness}

\section{Travel Time and Vehicle Throughput}

The mobility measures such as travel time and vehicle throughput are directly obtained from VISSIM. In the VISSIM network, the zone for evaluating the performance is defined by designating starting and ending point along the network. The travel time for each vehicle, the time taken for the vehicle to drive from the starting to the ending point, is averaged to estimate average travel time.

$$
T T=\frac{\sum_{i=1}^{N} T_{i}^{E n d}-T_{i}^{\text {Start }}}{N}
$$

Where: $\mathrm{TT}=$ average travel times $(\mathrm{sec})$

$$
\begin{aligned}
& T_{i}^{\text {Start }}=\text { time for a vehicle } i \text { reached to the starting point }(\mathrm{sec}) \\
& T_{i}^{\text {Start }}=\text { time for a vehicle } i \text { reached to the ending point }(\mathrm{sec}) \\
& N=\text { total number of vehicles }
\end{aligned}
$$

The vehicle throughput is counted number of vehicles that passes through the ending point during the time of evaluations.

\section{Fuel consumptions}

Fuel consumption measure is estimated using the polynomial meta-model proposed by Kamal et al. (2011).

$$
\dot{f}_{v}=\dot{f}_{\text {cruise }}+\dot{f}_{\text {accel }}
$$

Where: $\mathrm{t} \in \mathrm{R}^{+}$is the time 


$$
\begin{aligned}
& \dot{f}_{\text {cruise }}=\text { the fuel consumed by a vehicle traveling at a constant speed } v(t) \\
& \dot{f}_{\text {accel }}=\text { the additional fuel consumption caused by acceleration } u(t)
\end{aligned}
$$

The fuel consumption estimation models yield the estimation as a function of speed and acceleration such as

$$
\begin{aligned}
& \dot{f}_{\text {cruise }}=w_{0}+w_{1} \cdot v(t)+w_{2} \cdot v(t)^{2}+w_{3} \cdot v(t)^{3} ; \text { and } \\
& \dot{f}_{\text {accel }}=u(t) \cdot\left(n_{0}+n_{1} \cdot v(t)+n_{2} \cdot v(t)^{2}\right) .
\end{aligned}
$$

The polynomial coefficients of $w_{n}, n=0, \ldots, 3$ and $r_{m}, m=0,1,2$ are calculated from experimental data. In this dissertation, the coefficient values are used the same with the ones reported in a literature (Kamal et al. 2011).

For all simulation cases, 5 replications are produced to account for the effect of stochastic components of traffic and drivers' behaviors to ensure that the results produced statistically significant at a 95\% confidence level (VDOT 2013). The minimum sample size test is also performed considering the replicated data variances.

\section{Acceleration variations}

The measure of the acceleration variations is used to estimate the speed variations of a vehicle in a consecutive time. It is quantified as a sum square of the speed differences of the subject vehicle $i$ that normalized by the total number of vehicles $(N)$ used in the simulation. This measure shows the average magnitude of the vehicle speed fluctuations as the vehicle drives through the test-bed network. 
$\operatorname{AccVar}\left(f t / \mathrm{sec}^{2} / v e h\right)=\frac{\sqrt{\sum_{i=1}^{N}\left(V_{i, t}-V_{i, t-1}\right)^{2}}}{N}$

Equation (3)

Where: $V_{i, t}=$ speed of vehicle $i$ at time $t\left(\mathrm{ft} / \mathrm{sec}^{2}\right)$

$N=$ total number of vehicles

\subsubsection{Statistical Testing and Minimum Sample Size}

To ensure statistical significance of the results, the t-test is conducted for all comparing measurements by paring up of two scenarios out of the multiple scenarios if it is more than two. The statistical results showed that the p-values are close to zeros (rounded at the second decimal points), which implied that the measurement of certain scenario is significantly different from the other two scenarios at the 95 th percentile confidence level.

The integrity of the simulation results are further assessed by identifying the minimum sample size using the Sample Size Determination Tool (Version 2.0) which is developed based on the FHWA sample size determination methodology (VDOT 2013). In the tool, the minimum sample size is determined as a function of the statistics of the initial simulation runs, confidence level and the tolerance error. In this dissertation, the 95th percentile confidence interval and 5\% error tolerance value are used as suggested in the manual (VDOT 2013). The initial five runs of simulation satisfy the minimum sample size for all scenarios per the tolerance level of $5 \%$ with the 95 th percentile confidence level. The minimum sample size is summarized in Appendix 1-A through 1-C. In addition, given that the comparison evaluation involves multiple independent variables such as different control algorithms and CAV or AV market penetrations, the two-way Analysis of Variance (ANOVA) (Montgomery 2001) is conducted to investigate the individual influence of the 
two independent variables as well as the interaction influence of the two on each performance measures such as mobility and fuel consumptions measurements. 


\section{CHAPTER 4: DEVELOPMENT OF OPTIMAL CONTROL ALGORITHM}

This chapter describes the framework of the optimal control algorithm developed and evaluated in this dissertation. This chapter describes the process to formulate the framework of the optimal control strategies problem for both speed harmonization and traffic flow stabilization controls and describes mathematical process to obtain the analytical solution based on the closed-loop control system.

\subsection{OPTIMIZATION PROBLEM FORMULATION}

\subsubsection{Speed Harmonization Algorithm}

The basic idea on the SPD-HARM strategy of optimal control algorithm is to address the problem of controlling the speed of a number of automated vehicles before they enter a speed reduction zone on a freeway. We formulate the control problem and provide an analytical, closed-form solution that can be implemented in real time. The solution yields the optimal acceleration/deceleration of each vehicle under the hard safety constraint of rear-end collision avoidance.

In this algorithm, the vehicle dynamics of individual vehicle is defined as in Equation (4).

$$
\begin{aligned}
& \dot{x}_{i}=v_{i} \\
& \dot{v}_{i}=u_{i}
\end{aligned}
$$

Where: $x_{i}=$ travel distance of a vehicle $\mathrm{i}$ from a reference point (m)

$v_{i}=$ speed of a vehicle $\mathrm{i}(\mathrm{m} / \mathrm{s})$

$$
u_{i}=\text { acceleration of a vehicle } \mathrm{i} \text { (control input) }\left(\mathrm{m} / \mathrm{s}^{2}\right)
$$


When a vehicle enters the control zone of the controller, the controller determines a hierarchical vehicle sequence in order which is indexed by $i=1,2, \cdots n$, i.e., $x_{1}>x_{2}>x_{3}>\ldots>x_{n}$ as in Figure 2.

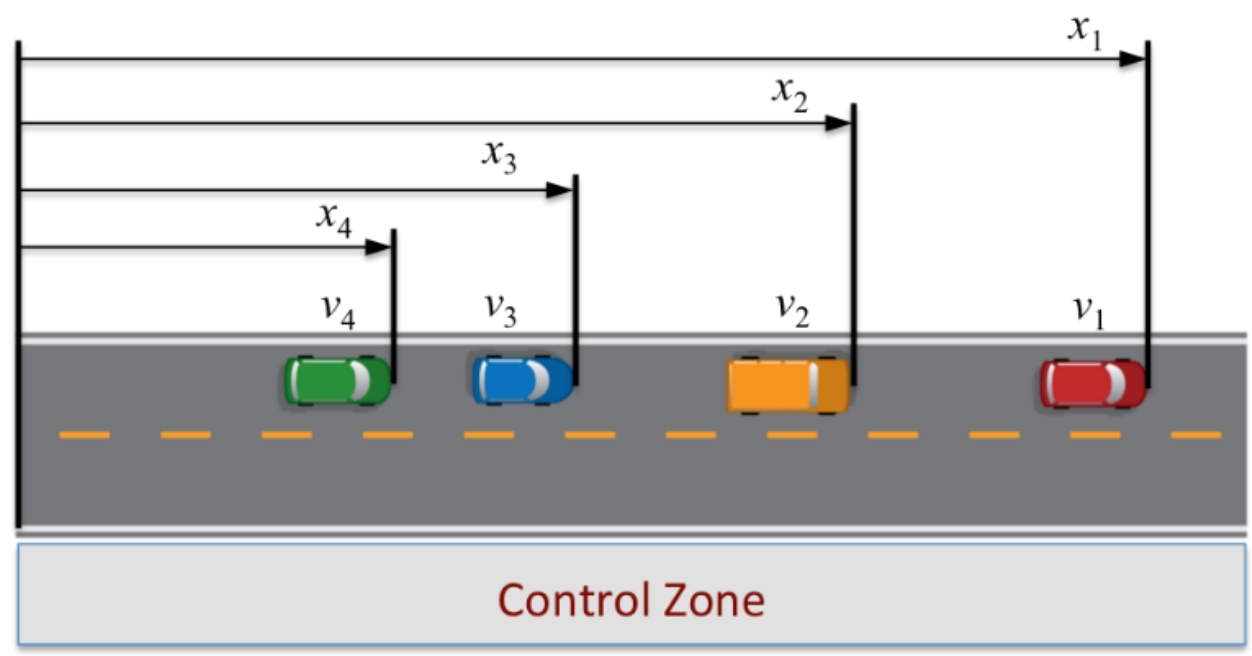

Figure 2. Hierarchical sequence of the vehicle in the control zone.

The optimal control aims at deriving the optimal acceleration profile for each vehicle when the leading vehicle, indexed by $i=1$, either accelerates or brakes between the interval of $\Delta t=t_{1}^{f}-t_{1}^{0}$, where $t_{1}^{0}$ and $t_{1}^{f}$ denotes the initial and final time for each of the vehicle $i$. The problem to optimize the acceleration of each vehicle $i$ can be formulated as Equation (5) subject to vehicle dynamics in Equation (4).

$$
\min _{u_{i}} J=\min _{u_{i}} \frac{1}{2} \sum_{i=1}^{n} \int_{t_{i}^{0}}^{t_{i}^{f}} u_{i}^{2} d t
$$

Where: $i=$ vehicle index

$$
\begin{aligned}
& n=\text { the number of vehicles in the control zone } \\
& u_{i}=\text { acceleration of a vehicle } i \text { (control input) }
\end{aligned}
$$


Subject to:

Vehicle Dynamics

$\dot{x}_{i}=v_{i}$

$\dot{v}_{i}=u_{i}$

Initial Conditions

$x_{i}\left(t_{i}^{0}\right)=0$

$v_{i}\left(t_{i}^{0}\right)=v_{i}$

Final Conditions

$x_{i}\left(t_{i}^{f}\right)=L$

$v_{i}\left(t_{i}^{f}\right)=v_{s r z}$

Where: $L=$ location of speed reduction zone

$v_{s r z}=$ speed limit of the speed reduction zone

The time $t_{i}^{f}$ that the vehicle $i$ will be entering the speed reduction zone is restricted by the imposing rear-end collision constraint. Therefore, to ensure that Equation (6) is satisfied at $t_{i}^{f}$ we impose the following condition:

$$
t_{i}^{f}=\max \left\{\min \left\{t_{i-1}^{f}+\frac{\delta}{v_{i-1}\left(t_{i-1}^{f}\right)}, \frac{L}{v_{\min }}\right\}, \frac{L}{v_{i}\left(t_{i}^{0}\right)}, \frac{L}{v_{\max }}\right\}
$$

Where: $v_{\max }=$ maximum speed for control

$$
\begin{aligned}
& v_{\text {min }}=\text { minimum speed for control } \\
& \delta \text { = safety minimum distance }
\end{aligned}
$$


In Equation (6), the $v_{i-1}\left(t_{i-1}^{f}\right)$ represents the speed of the vehicle $i-1$ at the time $t_{i-1}^{m}$ that enters the speed reduction zone, and it is equal to the speed imposed inside the speed reduction zone. Therefore, Equation (6) ensures that the time $t_{i}^{f}$ that vehicle $i$ will be entering the speed reduction zone is feasible and can attained based on the imposed speed limits inside the control zone. In addition, for the low traffic flow where vehicle $i-1$ and $i$ might be located far away from each other, there is no compelling reason for vehicle $i$ to accelerate within the control zone just to have safety distance $(\delta)$ from the preceding vehicle $i-1$ at the time $t_{i}^{f}$ that vehicle $i$ enters the speed reduction zone. Therefore, in such cases vehicle $i$ can keep cruising within the control zone with the initial speed that the vehicle $i$ entered the control zone at $t_{i}^{0}$.

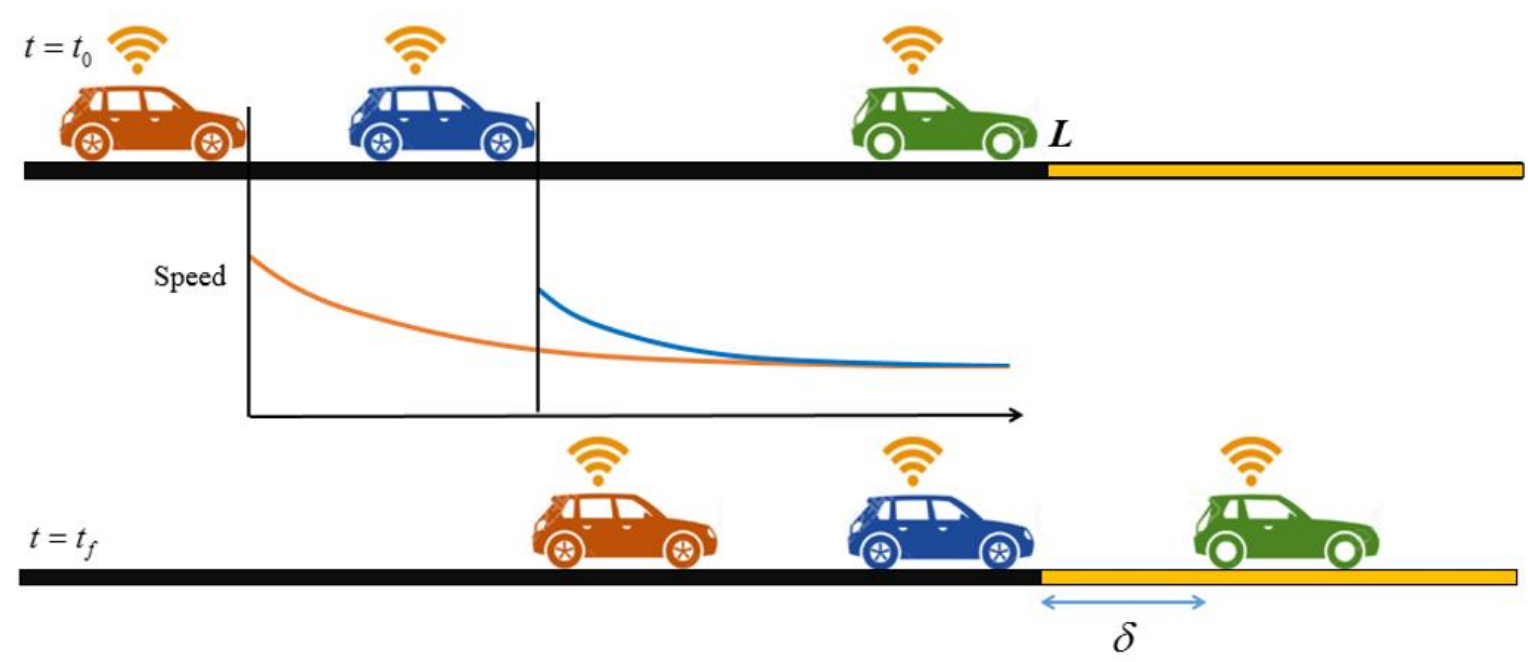

Figure 3. Schematic presentation of optimal control algorithm for speed harmonization 


\subsubsection{Traffic Flow Stabilization Control}

The basic idea of the traffic flow stabilization control is that the controlled vehicle $i$ is called to pursue or maintain a desired time headway from the preceding vehicle unless the trajectory violates the safety. When vehicle i initiate its control strategy, using the location and speed measurement of the preceding vehicle $i-1$, the vehicle $i$ identifies if the current condition signifies potential safety violations based on Equation (7).

$$
x_{i-1}\left(t_{0}\right)-x_{i}\left(t_{0}\right) \geq U_{d e s} \cdot h_{o p t}
$$

Where: $x_{i}(t)=$ location of vehicle $i$ at time $t$

$$
\begin{aligned}
& t_{0}=\text { time of current } \\
& U_{\text {des }}=\text { desired speed (or speed limit) } \\
& h_{\text {opt }}=\text { desired time headway }
\end{aligned}
$$

Basically, the Equation (7) represents the situation where the gap distance from the preceding vehicle ensures that the following vehicle can drive at the given desired speed to maintain the desired time headway assuming that both preceding vehicle and the subject vehicle sustain their current speed.

According to Equation (7), if condition is identified as positive, the final state of constraints are determined as:

$$
\begin{aligned}
& v_{i}\left(t_{f}\right)=\frac{x_{i-1}\left(t_{0}\right)-x_{i}\left(t_{0}\right)}{h_{o p t}}\left(\text { s.t. } v_{i}\left(t_{f}\right) \leq U_{d e s}\right) \\
& t_{f}=h_{o p t} \\
& x_{i}\left(t_{f}\right)=x_{i}\left(t_{0}\right)+\frac{\left(v_{i}\left(t_{0}\right)+v_{i}\left(t_{f}\right)\right)}{2} \cdot t_{f}
\end{aligned}
$$


Otherwise when the gap distance from the preceding vehicle is shorter than the expected travel distance upon the desired speed for the subject vehicle, then the vehicle $i$ determines its final state of constraints based on the anticipated distance and speed of the preceding vehicle to avoid rear-end collisions as shown in Equation (8). The safety distance is formatted as a function of the preceding vehicle speed $v_{i}\left(t^{f}\right)$. The future location and speed of the preceding vehicles are estimated using the vehicle dynamics equations assuming that the current acceleration or deceleration pattern is maintained for the control time interval.

$$
\begin{aligned}
& x_{i}\left(t_{f}\right)=x_{i-1}\left(t_{f}\right)-d_{\text {saft }} \\
& v_{i}\left(t_{f}\right)=v_{i-1}\left(t_{f}\right) \\
& t_{f}=\frac{x_{i}\left(t_{f}\right)-x_{i}\left(t_{0}\right)}{0.5 \cdot\left(v_{i}\left(t_{0}\right)+v_{i}\left(t_{f}\right)\right)}
\end{aligned}
$$

Where: $d_{\text {saft }}=v_{i}\left(t_{f}\right) \cdot h_{\text {opt }}$

$$
\begin{aligned}
& v_{i-1}\left(t_{f}\right)=v_{i-1}\left(t_{0}\right)+a_{i-1}\left(t_{0}\right) \cdot t_{\text {update }} \\
& a_{i-1}\left(t_{0}\right)=\frac{v_{i-1}\left(t_{0}\right)-v_{i-1}\left(t_{-1}\right)}{t_{\text {update }}} \\
& x_{i-1}\left(t_{f+1}\right)=x_{i-1}\left(t_{0}\right)+0.5 \cdot t_{\text {update }} \cdot\left(v_{i-1}\left(t_{-1}\right)+v_{i-1}\left(t_{0}\right)\right)
\end{aligned}
$$




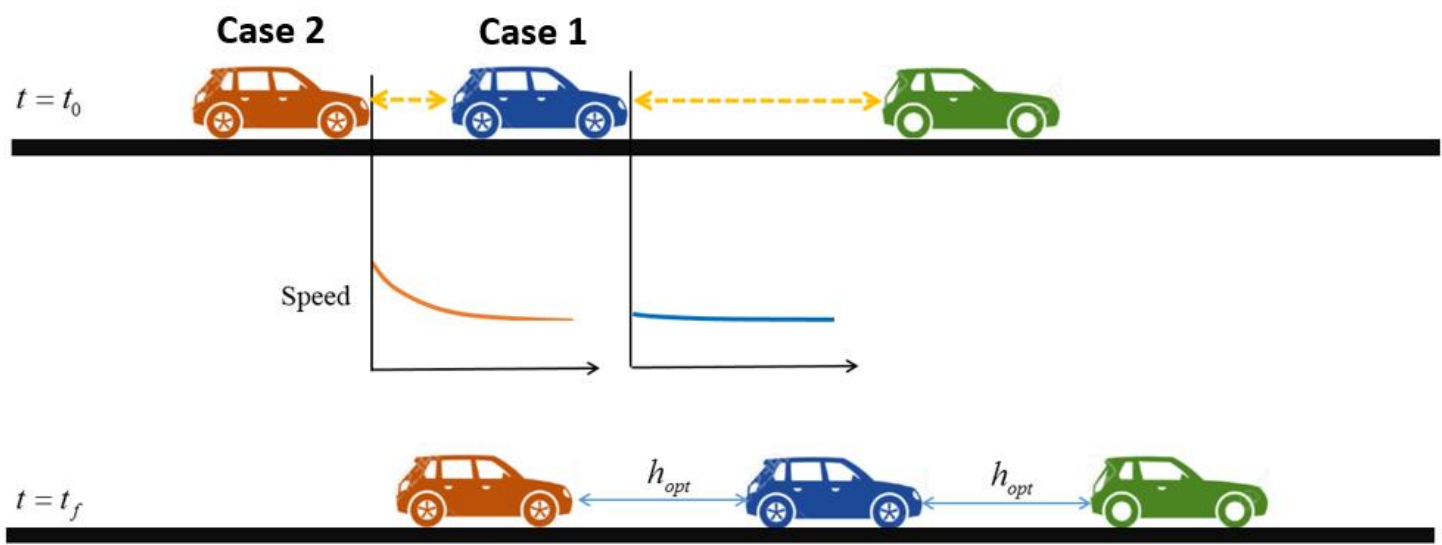

Figure 4 Schematic presentation of optimal control algorithm for traffic flow stabilization strategy.

\subsection{ANALYTICAL SOLUTION}

The Pontryagin's Minimum Principle (PMP) is applied to find the analytical closed-form solution for the problem forumlated in Equation (5) under the respective constraints of the SPD-HARM and traffic flow stabilization control. The problem is treated as a sequential minimization problem for each vehicle $i$ starting from the leading vehicle. The dynamics of the vehicle is described by an equation of the form $\dot{x}(t)=f(x(t), u(t), t)$. We seek to find the optimal control $u^{*}(t)$ which drives the system along an optimal trayectory $x^{*}(t)$.

For each vehicle $i$, the Hamiltonian function can be stated as Equation (9).

$$
H_{i}\left(\lambda_{i}^{x}, \lambda_{i}^{v}, x_{i}, v_{i}\right)=\frac{1}{2} u_{i}^{2}+\lambda_{i}^{x} v_{i}+\lambda_{i}^{v} u_{i}
$$

Where: $\lambda_{i}^{x}$ and $\lambda_{i}^{v}=$ the co-state components 
Applying the Hamiltonian minimization condition, the optimal control can be given as a function of the co-states as in Equation (10).

$$
u_{i}^{*}+\lambda_{i}^{v}=0
$$

The adjoining equations yield the following:

$$
\begin{aligned}
& \dot{\lambda}_{i}^{x}=-\frac{\partial H}{\partial x}=0 \\
& \dot{\lambda}_{i}^{v}=-\frac{\partial H}{\partial v}=-\lambda_{i}^{x},
\end{aligned}
$$

Hence $u_{i}^{*}=-\lambda_{i}^{v}$

From Equation (11) and (12), we have $\lambda_{i}^{x}=a_{i}$ that implies $\lambda_{i}^{v}=-\left(a_{i} t+b_{i}\right)$, where $a_{i}$ and $b_{i}$ are the constants of integration corresponding to each vehicle $i$. Consequently, the optimal control trajectory (acceleration profile) as a function of time is given as Equation (13).

$$
u_{i}^{*}=-a_{i} t+b_{i}
$$

Substituting Equation (10) to the vehicle dynamics equations in Equation (4), we can find the optimal speed and position for each vehicle, as shown in Equation (14) and (15).

$$
\begin{aligned}
& v_{i}^{*}(t)=\frac{1}{2} a_{i} t^{2}+b_{i} t+c_{i} \\
& x_{i}^{*}(t)=\frac{1}{6} a_{i} t^{3}+\frac{1}{2} b_{i} t^{2}+c_{i} t+d_{i}
\end{aligned}
$$

Where: $c_{i}$ and $d_{i}=$ constants of integration 
It is important to emphasize that this analytical solution allows the implementation of online optimal control that updates its optimality every control interval, which supports a dynamically changing traffic flow effectively. To derive online the optimal control policy (i.e., acceleration/ deceleration profile) for each vehicle, we need to update the constants at each control interval $\left(t_{f}\right)$. Equation (14) and (15) along with the initial and final conditions defined in the optimization problem in Equation (5) can be used to form a system of four equations of the form $\mathbf{T}_{\mathbf{i}} \mathbf{b}_{\mathbf{i}}=\mathbf{q}_{\mathbf{i}}$. It should be mentioned that initial and final conditions are already satisfied in this step.

$$
\left[\begin{array}{lccc}
\frac{1}{6}\left(t_{i}^{0}\right)^{3} & \frac{1}{2}\left(t_{i}^{0}\right)^{2} & t_{i}^{0} & 1 \\
\frac{1}{2}\left(t_{i}^{0}\right)^{2} & t_{i}^{0} & 1 & 0 \\
\frac{1}{6}\left(t_{i}^{f}\right)^{3} & \frac{1}{2}\left(t_{i}^{f}\right)^{2} & t_{i}^{f} & 1 \\
\frac{1}{2}\left(t_{i}^{f}\right)^{2} & t_{i}^{f} & 1 & 0
\end{array}\right]\left[\begin{array}{l}
a_{i} \\
b_{i} \\
c_{i} \\
d_{i}
\end{array}\right]=\left[\begin{array}{c}
x_{i}\left(t_{i}^{0}\right) \\
v_{i}\left(t_{i}^{0}\right) \\
x_{i}\left(t_{i}^{f}\right) \\
v_{i}\left(t_{i}^{f}\right)
\end{array}\right]
$$

The solution to the system can be calculated as in Equation (17).

$$
b_{i}=\left(T_{i}\right)^{-1} q_{i}
$$

Where: $\boldsymbol{b}_{\boldsymbol{i}}=$ a vector containing the four unknown constants $a_{i}, b_{i}, c_{i}$, and $d_{i}$.

Thus, as the constants are continuously updated using Equation (17), the controller yields the closed-loop optimal acceleration/deceleration for each vehicle $i$ over time. 


\section{CHAPTER 5: ESTABLISHMENT OF COMPARISON EXPERIMENTS}

This chapter describes the existing traffic behavior models and/or control algorithms that are used in comparisons experiments. Those state-of-the-art algorithms are evaluated in order to provide a baseline against which to compare all other existing control strategies with the proposed optimal control algorithm. In this research, the comparisons experiment considered (i) baseline of no-control and (ii) state-of-the-art algorithm(s) in the respective area. The baseline of no-control case is composed of $100 \%$ of human driven vehicles that follow psycho-physical car-following model (PTV 2014). In SPD-HARM strategy evaluations, two different types of SPD-HARM strategies are considered for the comparisons target: one is the Variable Speed Limit system - conventional technique of SPD-HARM, and the other is an individual vehicle-based SPD-HARM which premises that the controlled vehicles are equipped with the suitable sensor technique. In traffic flow stabilization control evaluations, the ACC algorithm (Milanés and Shladover 2014) is selected to compare with the proposed optimal control strategy that does not require communications between vehicles.

This chapter also describes on the procedures to implement each control algorithms under the microscopic simulation platform and some key factors to be noted.

\subsection{HUMAN DRIVERS MODEL}

The baseline of no-control associates with human drivers based on the Wiedemann 99 psycho-physical car-following model that is widely accepted as a good representation of perceptive behaviors of human drivers under uninterrupted traffic flow. 
To begin with, the VISSIM model is carefully calibrated by referring to the guideline of the Highway Capacity Manual (HCM) 2010 manual (TRB 2010). According to the HCM 2010, the capacity of two-lane highways under based conditions is identified as $1,700 \mathrm{veh} / \mathrm{hr}$. Without a possibility of having passing maneuvers from the opposite direction in the proposed test-bed network of the one-way corridor, a maximum flow rate of $1,800 \mathrm{veh} / \mathrm{hr}$ is considered appropriate to achieve through calibration. To this end, the key parameters for the car-following model which determine the minimum distance between adjacent vehicles are assessed during the calibration process. In VISSIM, the minimum safety distance $\left(d x_{\text {safe }}\right)$ which is defined as a distance a driver would maintain while following another vehicle can be expressed as shown in Equation (18) (PTV 2014).

$$
d x_{\text {safe }}=C C 0+C C 1 \cdot v
$$

Where: $C C O=\mathrm{a}$ standstill distance between two vehicles (in feet)

$C C 1=$ a headway time which a driver wants to maintain (in seconds) $v=$ average speed $\left(f t / \sec ^{2}\right)$

With a good amount of calibration effort, the $C C O$ is used as the default value of 4.92 feet and the $C C 1$ is adjusted to 1.2 seconds, thereby the maximum traffic flow is approximated about $1,800 \mathrm{veh} / \mathrm{hr}$ as desired.

To account for differences in the driving behavior of human drivers, VISSIM uses functions instead of individual input data. In this dissertation, the desired speed value is defined as normally distributed with an average value of the posted speed limit. The distribution functions of desired speeds used for each evaluation of speed harmonization 
and the traffic flow stabilization control are shown in Figure 5 and Figure 6, respectively. In those figures, the $\mathrm{x}$-axis represents the desired speed (mph) and the y-axis represents the proportional composition of vehicles in the range of 0 and 1 . The curve is adjusted to make a distribution close to normal distribution with the speed limit as an average of the distribution. In Figure 5, for example, about 35\% of vehicles run at the desired speed between 70 and $71 \mathrm{mph}$.

When there is a driver with a desired speed higher than that of the preceding vehicle, then it checks whether it can overtake other vehicles through lane changes while not violating safety (PTV 2014).

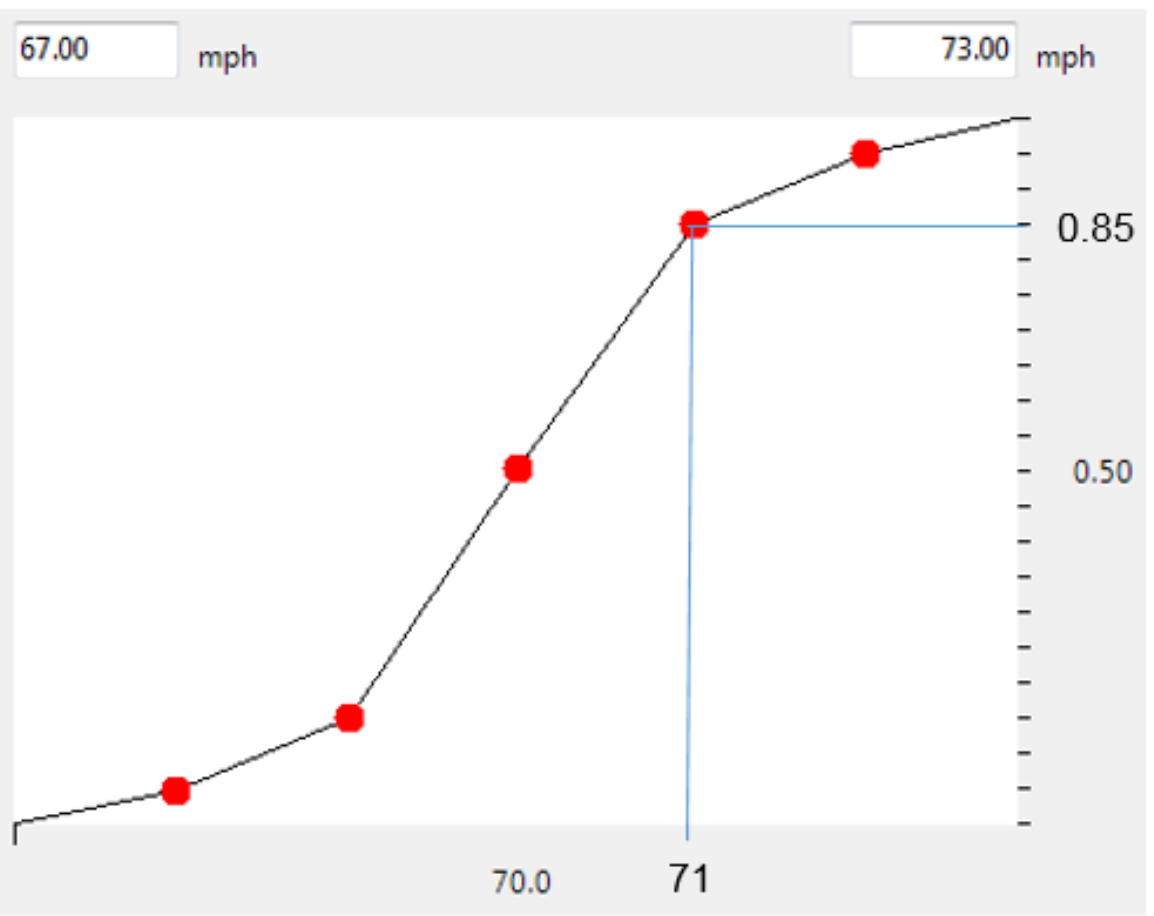

Figure 5. Speed distribution of human driven vehicles for speed harmonization evaluation under the speed limit of $70 \mathrm{mph}$. 


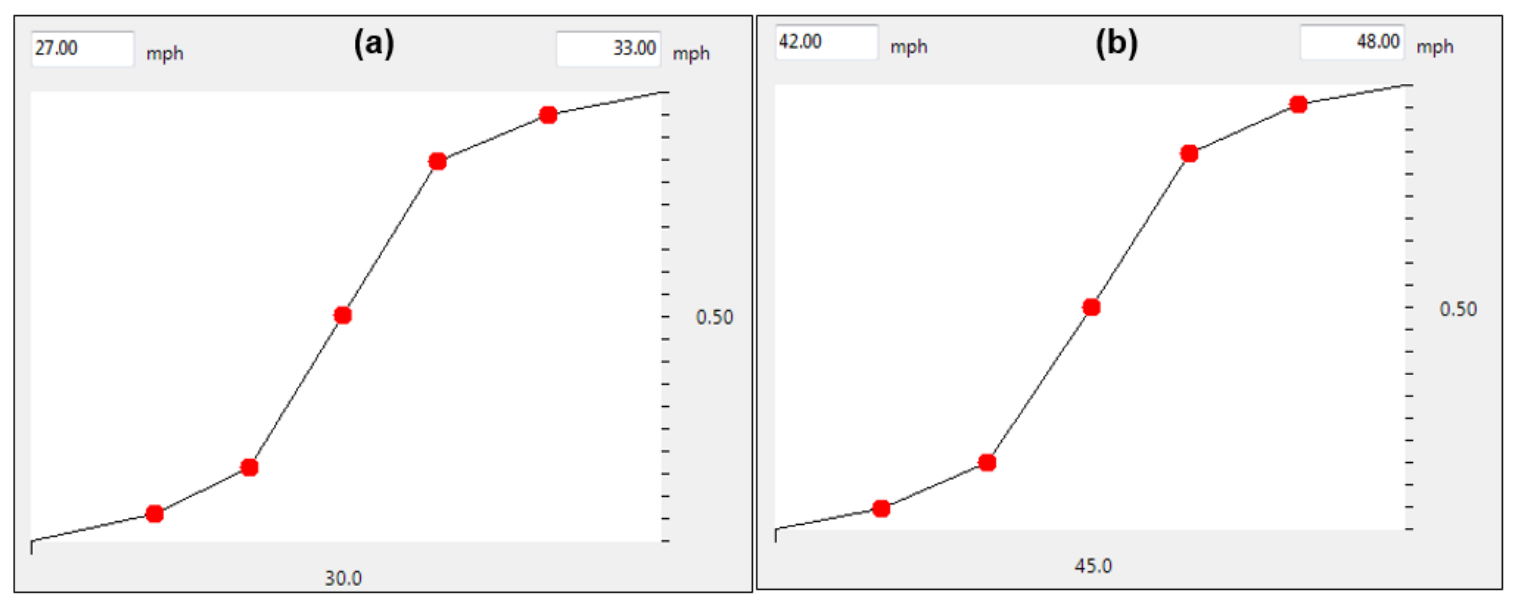

Figure 6. Speed distribution of human driven vehicles for traffic flow stabilization control evaluation under speed limit of (a) $30 \mathrm{mph}$ and (b) $45 \mathrm{mph}$, respectively.

\subsection{SPEED HARMONIZATION}

As mentioned in the introduction of this chapter, two types of SPD-HARM strategies that are most frequently adopted in practice (i.e., VSL system and the individual vehicle-based SPD-HARM system) are considered to compare with the proposed optimal control algorithm. By comparing with both SPD-HARM strategies, one can assess the potential benefits of the proposed algorithm over the current practice that has been validated through many other consecutive studies or field implementations; one can also learn about the benefits compared to the newly developed cutting-edge control algorithm although it may not be fully approved with its applicability in various situations.

Following sections summarize each of the two types of SPD-HARM strategies that are chosen in this research: (i) Speed Controlling Algorithm using Shock Wave Theory (SPECIALIST) (Hegyi et al. 2008); and (ii) the Simple Speed Harmonization algorithm developed as a federal projects in FHWA (Ma et al. 2016). 


\subsubsection{Speed Controlling Algorithm using Shock Wave Theory (SPECIALIST)}

The SPECIALIST is a proactive VSL algorithm that projects traffic conditions in the near future using the Model Predictive Control (MPC) method. This algorithm is considered as one of the most advanced since it utilizes shock wave theory to generate control speed and duration, and thus, it does not require complicated computation. In addition, it includes only a few parameters with physical interpretations for feasible field implementations.

The basic idea of the SPECIALIST lies on the transitions in fundamental relationship of traffic flow during the shockwave process. When the different traffic states

along the freeway are known, then their future evolution can be estimated by describing the location of fronts between the two different traffic flows. This basic concept is used to calculate the variable speed limit in a way to minimize the implantation duration and length of control. Details on the process can be found in the original paper (Hegyi et al. 2008).

In this dissertation, SPECIALIST is modeled using $\mathrm{C \#}$ programming and implemented in the VISSIM using its COM interface. Since SPECIALIST is based on a mesoscopic model that utilizes the spot-based measurement collected at a fixed location and aggregated for a certain period of time, detector stations are evenly embedded at every 75 meters along the corridor to estimate the local traffic states. The traffic state of each detector station are estimated every 60 seconds by using the aggregated estimation of the latest 60 seconds interval, and the activation of VSL are examined every 60 seconds as well. The resultant speed control policy is applied as a feedforward control to the traffic process. Once the control speed and duration are generated, a new measurement is not updated until the current control is finished. 
The SPECIALIST algorithm has several parameters that can be selected by the operator. For the best performance of the algorithm, the parameters are tuned with several iterations. The thresholds of maximum speed and capacity are chosen as $15.6 \mathrm{~m} / \mathrm{s}$ and 1,500 $\mathrm{veh} / \mathrm{h}$, respectively, which are determined after empirical trials to find the minimum values where traffic congestion is not observed under the VSL implemented at the $100 \%$ automated vehicle market penetration.

\subsubsection{Simple Speed Harmonization}

A vehicular-based SPD-HARM algorithm, called Simple Speed Harmonization (SH) Algorithm (Ma et al. 2016) was developed through the support of US DOT in an effort to realize field implementation of SPD-HARM algorithms using connected and automated vehicles. The simple SPD-HARM algorithm was created in inspiration from the work of Lu et al. (2014). However, the Simple Speed Harmonization algorithm was the simplified version that was designed to demonstrate the feasibility under the context of probe-based traffic management using CAVs. The control logic is represented in Equation (19).

$$
\begin{aligned}
& s_{i}(t)=m(t) \cdot x_{i}(t)+b(t) \\
& m(t)=\frac{s_{i}(t)-s_{j}(t)}{\Delta x_{i j}} \\
& b(t)=s_{i}(t)
\end{aligned}
$$

Where:

$s_{i}(t)=$ speed of vehicle $i$ at time $\mathrm{t}$

$x_{i}(t)=$ location of vehicle $i$ at time $\mathrm{t}$. 
With successful validation of field implementation (Ma et al. 2016), the effectiveness of the Simple Speed Harmonization algorithm in mitigating the traffic oscillations has been updated by Learn (2016). The recent results showed that the vehiclebased SPD-HARM algorithm effectively mitigated the traffic oscillations compared to the base case of no-control, while the travel time and fuel consumption are increased over the base case (Learn 2016).

\subsection{TRAFFIC STABILIZATION STRATEGY}

To evaluate the performance of the traffic stabilization strategy of optimal control algorithm, we considered the Adaptive Cruise Control (ACC) algorithm as the most comparable control scheme since the ACC strategies aim at minimizing the control efforts of individual vehicle in the context of vehicle platoon by stabilizing the headway gap, but still does not require communication technique unlike the Cooperative Adaptive Cruise Control (CACC). In this research, the ACC algorithm developed by Milane and Shladover (2014) is selected since the ACC algorithm is one of the few traffic flow stabilization controls that validated its results through the field test (Milanés and Shladover 2014).

\subsubsection{ACC algorithm of Milane and Shladover}

This ACC algorithm was developed as an improvement of the previous model presented in Shladover et al. (2012) by exploiting the experimental results for modeling the ACC algorithm. The maximum acceleration and deceleration set for the control algorithm are $1 \mathrm{~m} / \mathrm{s}^{2}$ and $2.8 \mathrm{~m} / \mathrm{s}^{2}$ respectively - as defined in the original research (Milanés 
and Shladover 2014), which are determined based on the real vehicle limitations. As shown in Equation (20), the vehicle acceleration is a function of the distance and speed errors.

$$
a_{k}=k_{1}\left(x_{k-1}-x_{k}-t_{h w} \cdot v_{k}\right)+k_{2}\left(v_{k-1}-v_{k}\right)
$$

Where: $a_{k}=$ acceleration of the $\mathrm{k}$-th vehicle

$$
\begin{aligned}
& x_{k}=\text { current position of the preceding and the subject vehicle } \\
& v_{k}=\text { current speed of the preceding and the subject vehicle } \\
& t_{h w}=\text { current time-gap setting } \\
& k_{l}=\text { gains on positioning error } \\
& k_{2}=\text { gains on speed error }
\end{aligned}
$$

The $k_{1}$ and $k_{2}$ values used in this study are $0.23 \mathrm{~s}^{-2}$ and $0.07 \mathrm{~s}^{-2}$, respectively, which are resulted to produce minimum optimization criterion value based on the Integral Absolute Error (IAE) as expressed in Equation (21).

$$
I A E=\int_{0}^{T}\left(\left|v_{\text {real }}-v_{\text {simulated }}\right|\right) d t
$$

Where: $v_{\text {real }}=$ speed of the real vehicle

$$
v_{\text {simulated }}=\text { speed of the vehicle in simulation }
$$




\section{CHAPTER 6: DEVELOPMENT OF MICROSCOPIC SIMULATION PLATFORM}

\subsection{ADVANCED MICROSCOPIC TRAFFIC SIMULATION TOOL}

This chapter describes the advanced microscopic traffic simulation tool that is created to evaluate the effectiveness of the proposed optimal control algorithm. Figure 7 illustrates the overall architecture of the advanced traffic simulation tool. The optimal control algorithm is coded using MATLAB language Dynamic Link Library (DLL) interface programming to allow data exchange with other external programs within the framework. A simulation test-bed networks are developed under VISSIM, and they are integrated into the framework by using its COM interface.

The beauty of the simulation platform developed in this research is that it is capable to emulate the interactions between the two different agents with distinctive driving behavior patterns, i.e., human driven vehicles and automated vehicles. Such capability is credited to the simulation platform which integrates a controller and a simulator upon Visual C\# programming environment, and allows to exchange information fed back into each side of component real-time. 


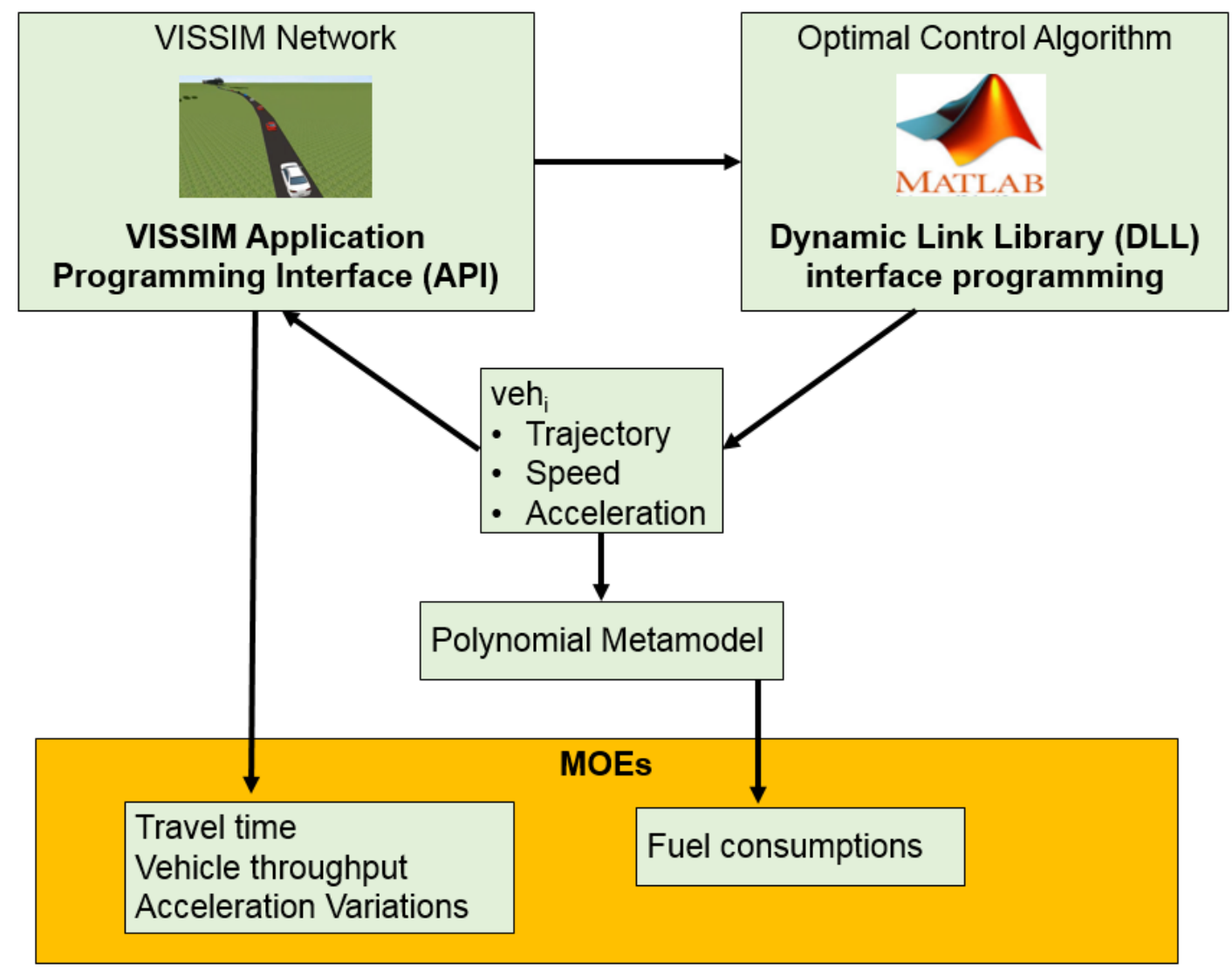

Figure 7. Overview of simulation framework.

The assumptions rest in this simulation operation are:

- There is no human interventions in the control of connected and automated vehicles

- The connected and automated vehicles are fully operated with no malfunctions or sensor errors;

- All connected and automated vehicles are equipped with radar sensors which can collect trajectory information of preceding vehicles real-time, and information from the road-side units to collect speed limit policy, but it does not the communication between other vehicles; and 
- Vehicles are perfectly controlled by the optimal control algorithm, but not governed by other factors or authority of control

\subsection{TEST-BED NETWORK}

\section{Speed Harmonization}

A hypothetical test-bed network for implementation of the SPD-HARM is developed and calibrated in the process described in Chapter 5.1. The network consists of the 7,000-feet long single lane corridor without any on-/off- ramps as shown in Figure 8. The speed limit of the corridor is set as $70 \mathrm{mph}$, and a speed reduction zone of $35 \mathrm{mph}$ is located at the 5,000-feet down from the upstream.

The control algorithms tested in this research are implemented within the fixed length of control zone which is located immediate upstream the speed reduction. The length of the control zone is used as 300 meters which is enough to cover the length of the possible traffic congestions which occurs due to the adjacent speed reduction zone.

For the simulation runs, the total simulation period is 1,000 seconds which is comprised of 100-second warm-up period and 900-second of algorithm implementation. The warm-up period is necessary to avoid empty network situation during the algorithm applications. 


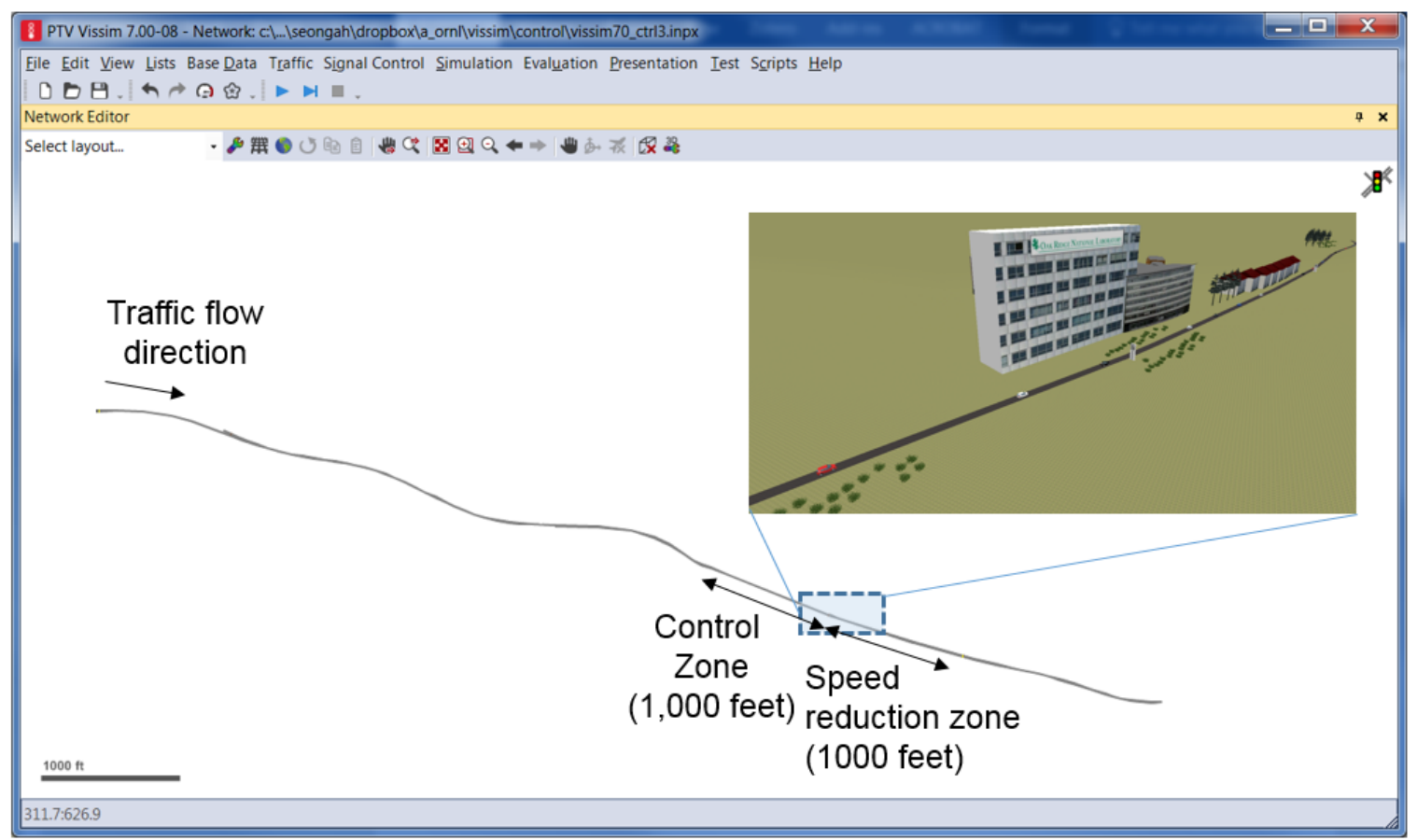

Figure 8 VISSIM testbed for speed harmonization

\section{$\underline{\text { Traffic Flow Stabilization }}$}

A testbed network for testing the traffic flow stabilization control consists of a 21,000-feet long 3-lane corridor that does not have on-/off- ramps as shown in Figure 9. It has to be mentioned that this network premises a straight roadway for the entire length, and any impacts from the curves on the driving behavior - such as a speed reduction on the curve is completely exempted through the modeling process in VISSIM.

The prevailing speed limit of the roadway is $30 \mathrm{mph}$ but there is a roadway section where the speed limit increases to $45 \mathrm{mph}$ for the next 700 feet, and then the speed limit changes back to $30 \mathrm{mph}$ for the next 700 feet as illustrated in Figure 9.

The tested control algorithms are implemented throughout the entire corridor on to the controlled vehicles. For the purpose of evaluating the impact of stabilizing the traffic flow in the car-following process, it is unnecessary to employ warming-up period to load 
vehicles and observe prevailing traffic patterns in the network. Therefore, instead of releasing traffic flow into the network, we positioned all vehicles with a constant spaces of the desired time headway and let them start driving at the desired speed limit with the initiation of the simulation as shown in the 3-D representation in Figure 9.

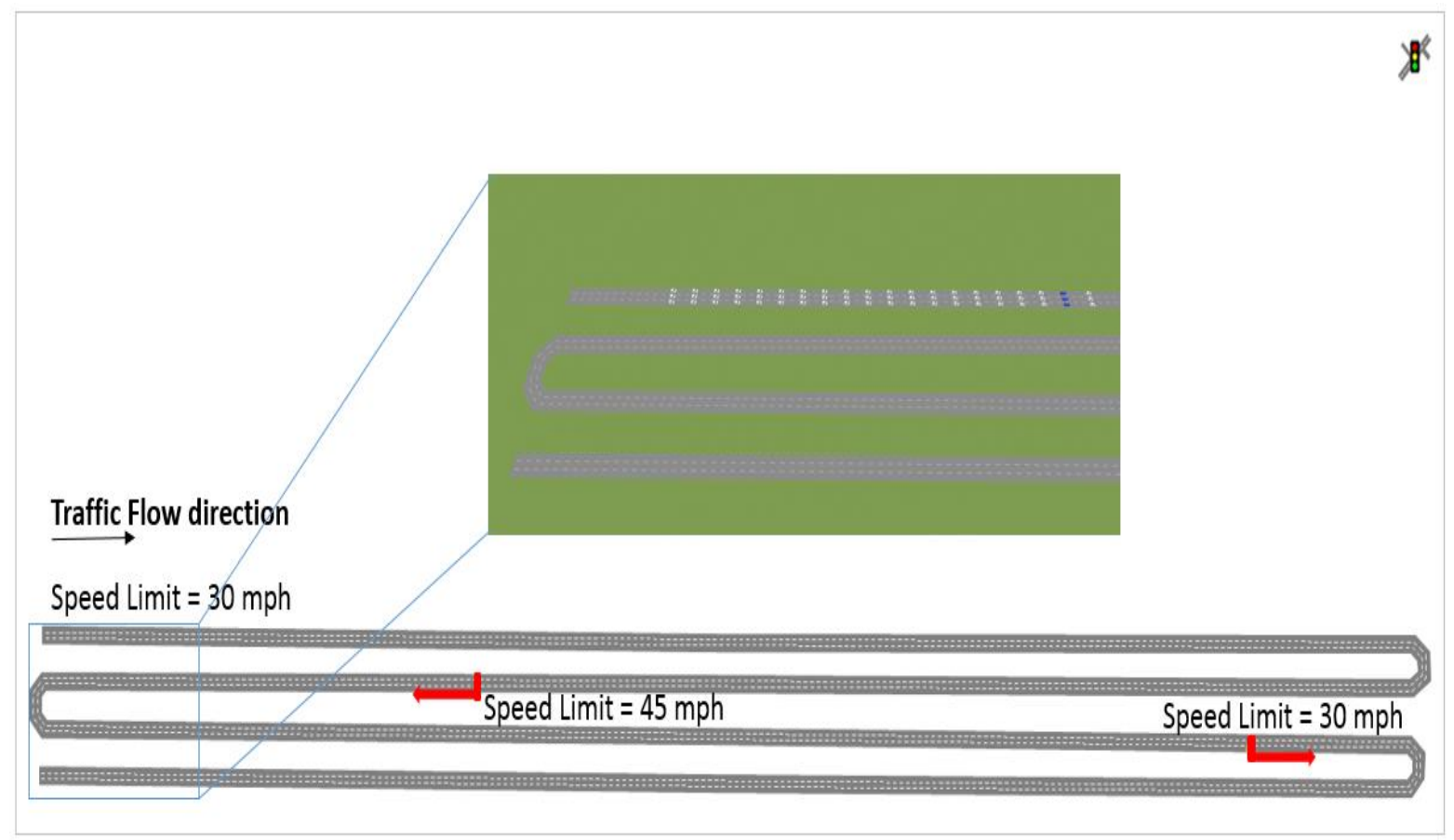

Figure 9. VISSIM testbed for traffic flow stabilization control.

There are 60 vehicles used for the test and there is no additional traffic input during the simulation. At starting of the simulation, 20 vehicles are positioned for each lane. Their gap distance at the initiation is about 53 feet which is calculated by multiplying the desired speed and the desired time headway gap (i.e., $44 \mathrm{fps}($ or $30 \mathrm{mph}) \times 1.2$ seconds $=52.8$ feet $)$.

In addition, the human driven vehicles are allowed to change lanes as it is ruled by the Wiedmann car-following logic, while the automated vehicles are not permitted to do lane-change for the cases of all control algorithms tested in this study due to the lack of the logics integrated into the traffic stabilization strategy at this stage. 


\section{CHAPTER 7: RESULTS AND ANALYSIS}

This chapter discusses the results of the SPD-HARM and the traffic flow stabilization controls in comparisons with the baseline case of human drivers as well as the existing state-of-the-practices. Sensitivity on the performance of the algorithm with respect to the traffic volumes and the market penetrations of automated vehicles are conducted. The control algorithms are assessed from various perspectives such as mobility and traffic flow stability (speed variations), and fuel consumptions which are chosen according to the purpose and the context of the control scheme. The following chapters provides the results and analysis in detail.

\subsection{SPEED HARMONIZATION}

\subsubsection{Under the perfect market penetrations of CAVS}

This section discusses the results of fuel consumption, travel time, and throughput of the three approaches such as the existing VSL system (i.e., SPECIALIST algorithm), vehicular-based SPD-HARM algorithm (i.e., Simple SPD-HARM algorithm), and the proposed optimal speed control algorithm. Summary of the results is represented in plot of Figure 10. 


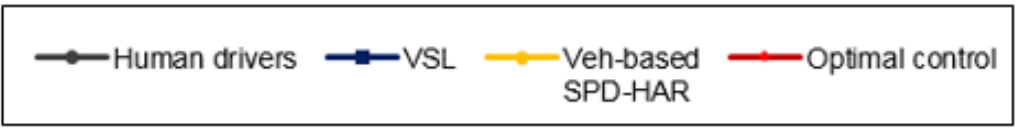

(a) 28

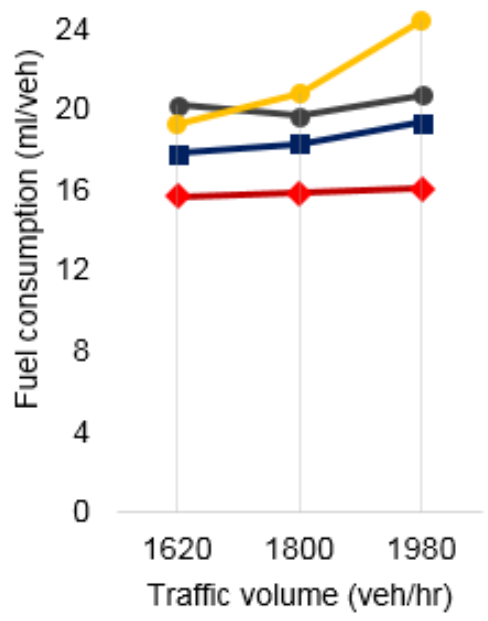

(b) 30

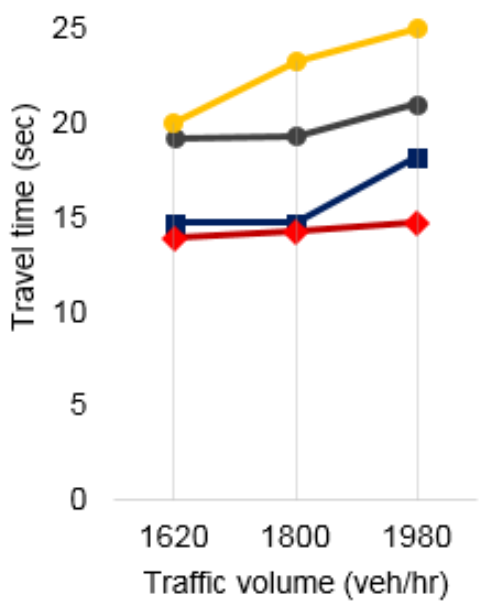

(c) 400

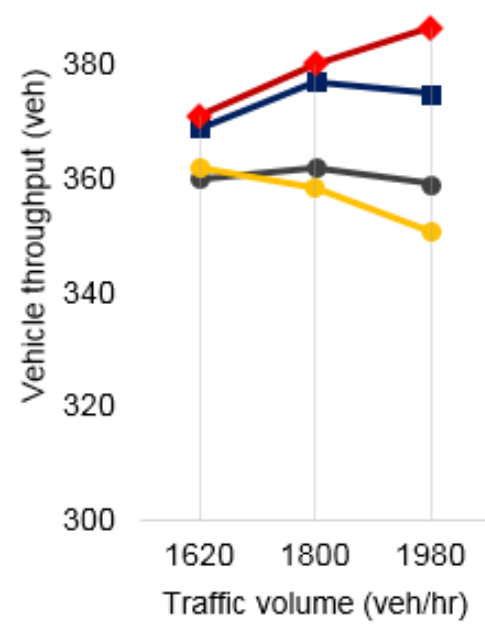

\section{Figure 10. Performance of SPD-HARM algorithms under varying traffic volumes}

\section{under $100 \%$ of AVs}

\section{$\underline{\text { Mobility }}$}

As shown in Figure 10-(b) and (c), the optimal control algorithm improves travel time and throughput for all traffic volumes over the baseline scenario, VSL, and the vehicular-based SPD-HARM algorithm. In particular, travel time is improved by $26-30 \%$ over the baseline scenario, by $3-19 \%$ over the VSL algorithm, and by $31-39 \%$ over the vehicular-based SPD-HARM algorithm for the three traffic volume cases. Both VSL and the proposed control algorithm reduces the travel time and improved the vehicle throughput under all three traffic volume cases. The proposed control algorithm reduces travel time and throughput by $19 \%$ and $3 \%$ respectively, compare to VSL under the traffic volume higher than the capacity. 
On the contrary, the vehicular-based SPD-HARM increases travel compared to the baseline scenario. This is a reasonable outcome since the vehicular-based SPD-HARM is designed to lead harmonizing speed throughout the traffic stream, but not optimizing its performance. However, the vehicle throughput at the bottleneck area is not significantly different than those of baseline scenario when the traffic volume is less than the capacity or at the capacity at 95\% confidence level as shown in Figure 10-(c). This implies that even though the vehicular-based SPD-HARM may increase the average travel time along the control zone, it eventually results in resolving potential shockwaves at the downstream bottleneck area and helped release the vehicles effectively unless the traffic congestion is initiated.

It is important to highlight that the proposed optimal speed control algorithm does not require a phase to clear congestion or a technique to prevent the bottleneck formation in order to improve vehicle throughput. Instead, the vehicle throughput is improved by having all individual vehicles proactively determines their optimal trajectories to the target location while the minimum spacing from the preceding vehicle is assured.

\section{Fuel consumptions}

Fuel consumption per vehicle for the three approaches demonstrate similar patterns with the results of travel time as shown in Figure 10-(a) and (b). By optimizing the vehicles' acceleration/deceleration inside the control zone, the time of reaching the speed reduction zone is controlled optimally, thus the recovery time from the congested area is minimized as shown in Figure 10-(b). In addition, each vehicle avoids getting into a stop-and-go driving mode, thereby conserving momentum and energy. Eliminating the vehicles' stopand-go driving aims at minimizing transient engine operation, and thus we have direct 
benefits in fuel consumption, as shown in Figure 10-(a), since internal combustion engines are optimized over steady state operating points (constant torque and speed) (Rios-Torres and Malikopoulos 2016)

The proposed optimal speed control algorithm significantly reduces fuel consumption of each vehicle by $19-22 \%$ over the baseline scenario, by $12-17 \%$ over the VSL algorithm, and by $18-34 \%$ over the vehicular-based SPD-HARM algorithm for the three traffic volume cases considered in this research.

As for the vehicular-based SPD-HARM, fuel consumption is statistically not different from that of the baseline scenario for the traffic volume case of road capacity and the case of $10 \%$ less than capacity. When the traffic volume is $10 \%$ higher than the capacity, fuel consumption increases by $18 \%$ over the baseline scenario. These observations are consistent with the results in the experiment conducted in the research group of FHWA (Learn 2016). 


\subsubsection{Under mixed traffic of CAVs and HDVs}

This section summarizes the comparison results among the optimal control algorithm, the two types of state-of-the-art VSL algorithms, and the base case of human drivers under varying automated vehicle market penetrations. It is noted that the base case of human drivers is specified as the case with zero percentage automated vehicle market penetration which is located in the left-end column of Figure 11 through Figure 13.

\section{Mobility}

Mobility performance is assessed based on the travel time and the number of vehicle throughput within the control zone. Figure 11 and Figure 12 show the results of travel time and vehicle throughput respectively among the base case, two types of SPDHARM algorithms and the optimal control algorithm.

In general, the travel time shows distinctive patterns among the three types of SPD-HARM algorithms as the market penetrations of CAV varies. Under the $\mathrm{V}$ implemented, the travel time increases by up to $22 \%$ when the CAV penetration is $50 \%$, and at least $70 \%$ of market penetration is required to outperform the base case. The increase in the travel time compared to the base case under low market penetrations is plausible result, because any SPD-HARM may increase the travel time, since it most likely to reduce the speed of the vehicle in the upstream earlier than what the vehicle would have driven under no-control situation. The market penetration of AV controlled by the VSL algorithm is even low, therefore the effects of speed management not in effect eventually. However, as higher the market penetration of the CAVs, it eventually facilitates smooth driving through the control zone and improves the travel time. 
When the vehicle-based SPD-HARM is implemented, the travel time constantly increases with the increase in the market penetrations of CAVs and levels off at the $60 \%$ of penetrations. It is reasonable to observe that the vehicle-based SPD-HARM rather increases travel time than the base case of human drivers even at higher penetrations of CAVs, because the vehicle-based SPD-HARM is essentially designed to lead harmonizing speed throughout the traffic stream, but not optimizing its mobility performance. However, as shown in Figure 12, the vehicle throughput at the bottleneck area is not significantly different than those of base case and the section-based SPDHARM at 95\% confidence level. This implies that even though the vehicle-based SPDHARM may increase the average travel time along the control zone, it eventually results to resolve potential shockwaves at the downstream bottleneck area and helped release the vehicles effectively.

On the contrary, the optimal control algorithm constantly reduces the travel time as the market penetration rate increases. The improvement in the travel time is gained by applying the constraint of a minimum safety distance in the optimization model, while the control solution for individual vehicle generates smooth trajectories to pass through the bottleneck area to result in the effect of SPD-HARM. Even under the low penetrations of $\mathrm{AVs}$, the adjacent following human driven vehicle not necessarily make sudden decelerations, but closely follow the preceding CAV to have the significant improvement. As shown in Figure 11, the vehicle throughput is improved by $8 \%$ over the base case, and it is maintained throughout different market penetrations.

Eventually, at $100 \%$ market penetrations, the travel time reductions for each three types of algorithm compared with the base case of human driven vehicles are $-11 \%, 20 \%$ 
and $-28 \%$ for the VSL algorithm, the vehicle-based SPD-HARM and the optimal control algorithm, respectively.

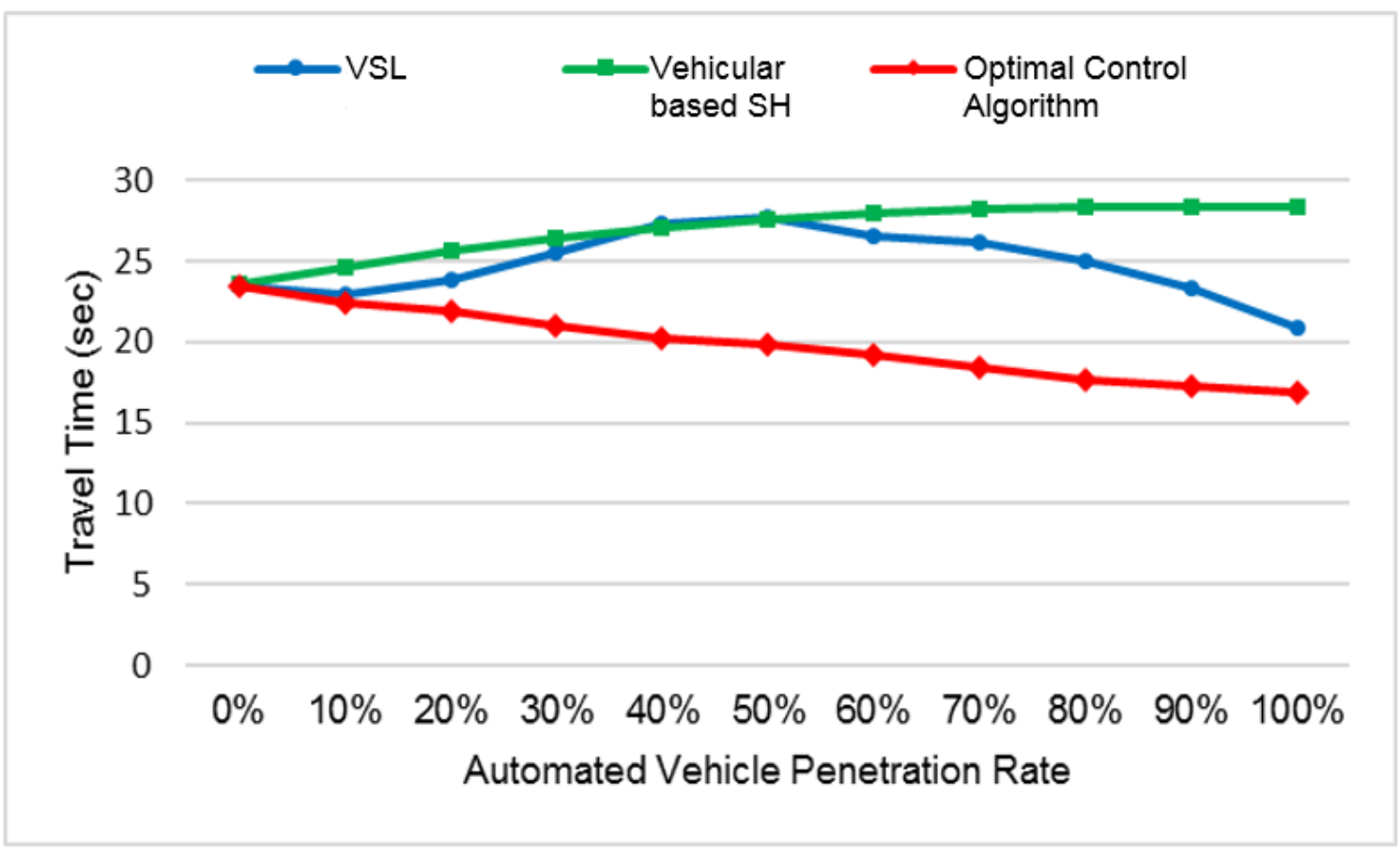

Figure 11. Travel time evaluations under varying penetrations of CAV

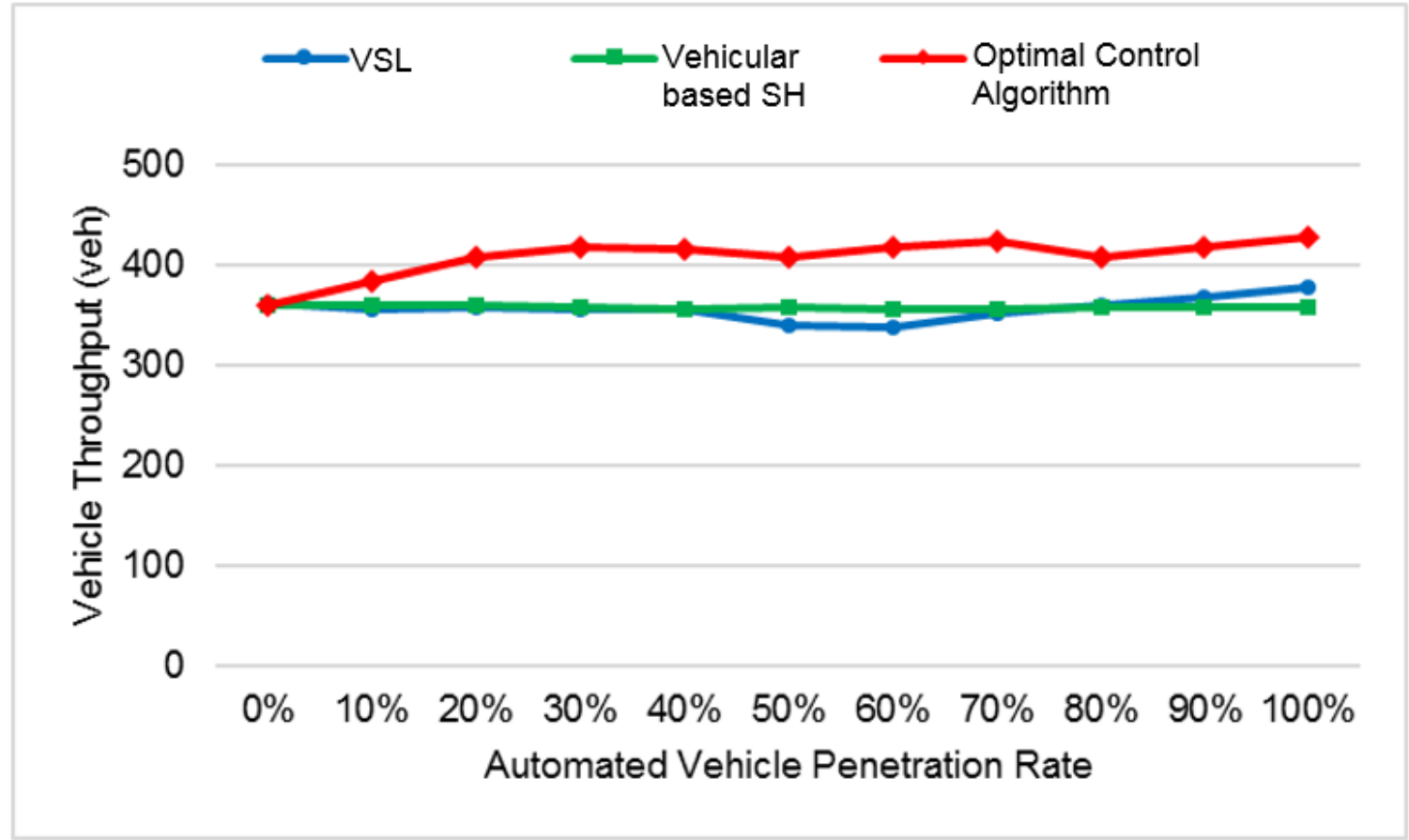

Figure 12. Vehicle throughput under varying penetrations of AVs 
To investigate the statistical significance of the individual impact of each factor such as the control algorithm and the CAV market penetration level as well as the interaction impact of those two factors, the ANOVA analysis is conducted. As shown in Table 2 and Table 3, the impacts of each main factor on the travel time and the vehicle throughput were significant at the 0.05 significance level. That is, the travel time and the vehicle throughput under the optimal control algorithm were significantly improved compared to those of both VSL and the vehicle-based SPD-HARM algorithms. In addition, the different levels of CAV market penetrations itself generated significant impacts on the travel time and the vehicle throughput, i.e., the travel time and the vehicle throughput increased with the higher level of CAV penetrations across different control algorithms.

The influence of the interaction of the two factors were shown to be significant for all performance measures, by yielding the F-values of 8.5 (p-value $<0.001$ ) for travel time and the F-values of 161.5 ( $\mathrm{p}$-value < 0.001) for vehicle throughput. This results infers that the impacts of CAV market penetrations on the mobility measures are different depending on the control algorithms. The improvements in travel time and vehicle throughput were greater under the optimal control algorithm than under the VSL algorithm or Vehicle-based SPD-HARM algorithms.

Table 2 ANOVA Result for Travel Time

\begin{tabular}{|l|c|c|c|c|c|}
\hline \multicolumn{1}{|c|}{ Source } & $\begin{array}{c}\text { Sum of } \\
\text { Squares }\end{array}$ & df & $\begin{array}{c}\text { Mean } \\
\text { Square }\end{array}$ & F & Significance \\
\hline (A) Control Algorithm & 1623.19 & 2 & 811.59 & 192.40 & 0.00 \\
\hline (B) CAV Market Penetration & 247.32 & 10 & 24.73 & 5.86 & 0.00 \\
\hline (A) $($ B) & 721.17 & 20 & 36.05 & 8.54 & 0.00 \\
\hline Error & 1252.80 & 297 & 4.21 & - & - \\
\hline Total & 3844.49 & 329 & - & - & - \\
\hline
\end{tabular}


Table 3 ANOVA Result for Vehicle Throughput

\begin{tabular}{|l|c|c|c|c|c|}
\hline \multicolumn{1}{|c|}{ Source } & $\begin{array}{c}\text { Sum of } \\
\text { Squares }\end{array}$ & df & $\begin{array}{c}\text { Mean } \\
\text { Square }\end{array}$ & F & Significance \\
\hline (A) Control Algorithm & 7493.99 & 2 & 3746.99 & 1105.76 & 0.00 \\
\hline (B) CAV Market Penetration & 7323.68 & 10 & 732.368 & 216.125 & 0.00 \\
\hline (A) $*$ B) & 10942.3 & 20 & 547.114 & 161.456 & 0.00 \\
\hline Error & 1006.42 & 297 & 3.38863 & - & - \\
\hline Total & 26766.4 & 329 & - & - & - \\
\hline
\end{tabular}

\section{Fuel consumption}

Figure 13 shows the per-vehicle fuel consumption among different SPD-HARM algorithms. It is arguable that the per-vehicle fuel consumption within the control zone is likely to, but not always, relate to travel time; less time a vehicle takes to drive through the control zone, less fuel is consumed for a vehicle, and vice versa. The general trend of fuel consumptions for the three types of SPD-HARM algorithms shows somewhat comparable patterns with that of travel time for each SPD-HARM algorithm.

When the VSL algorithm is implemented, the fuel consumption is significantly increased compared to the base case under the market penetrations of $50 \%$ to $70 \%$. It can be recalled that the number of conflicts is significantly high within the penetrations range of $50 \%$ to $70 \%$, which implies that the frequent speed changes and aggressive driving behaviors are occurred more often, and such driving behaviors also factors in the increase in the fuel consumptions. The per-vehicle fuel consumption turns to decline as the penetration rate is greater than $70 \%$, which is consistent with the pattern of travel time for the VSL algorithm. Eventually, the fuel consumptions becomes lower than that of the base case at $100 \%$ market penetrations by $-5.7 \%$.

With the vehicle-based SPD-HARM implemented, the fuel consumption is maintained from the level of the base case of human driven vehicles throughout varying 
market penetrations of AVs. This results are consistent with the result in the previous experiment (Learn 2016).

Under the optimal control algorithm implemented, on the contrary, the per-vehicle fuel consumption is reduced from that of the base case throughout the varying market penetrations of CAVs. The optimal control algorithm reduced the fuel consumptions thanks to the control solution that performs the minimization of the acceleration variations which directly relates to fuel consumption. Under $100 \%$ of $\mathrm{CAV}$, the fuel consumption reduction rate is maximized as about $-21 \%$ compared with the base case.

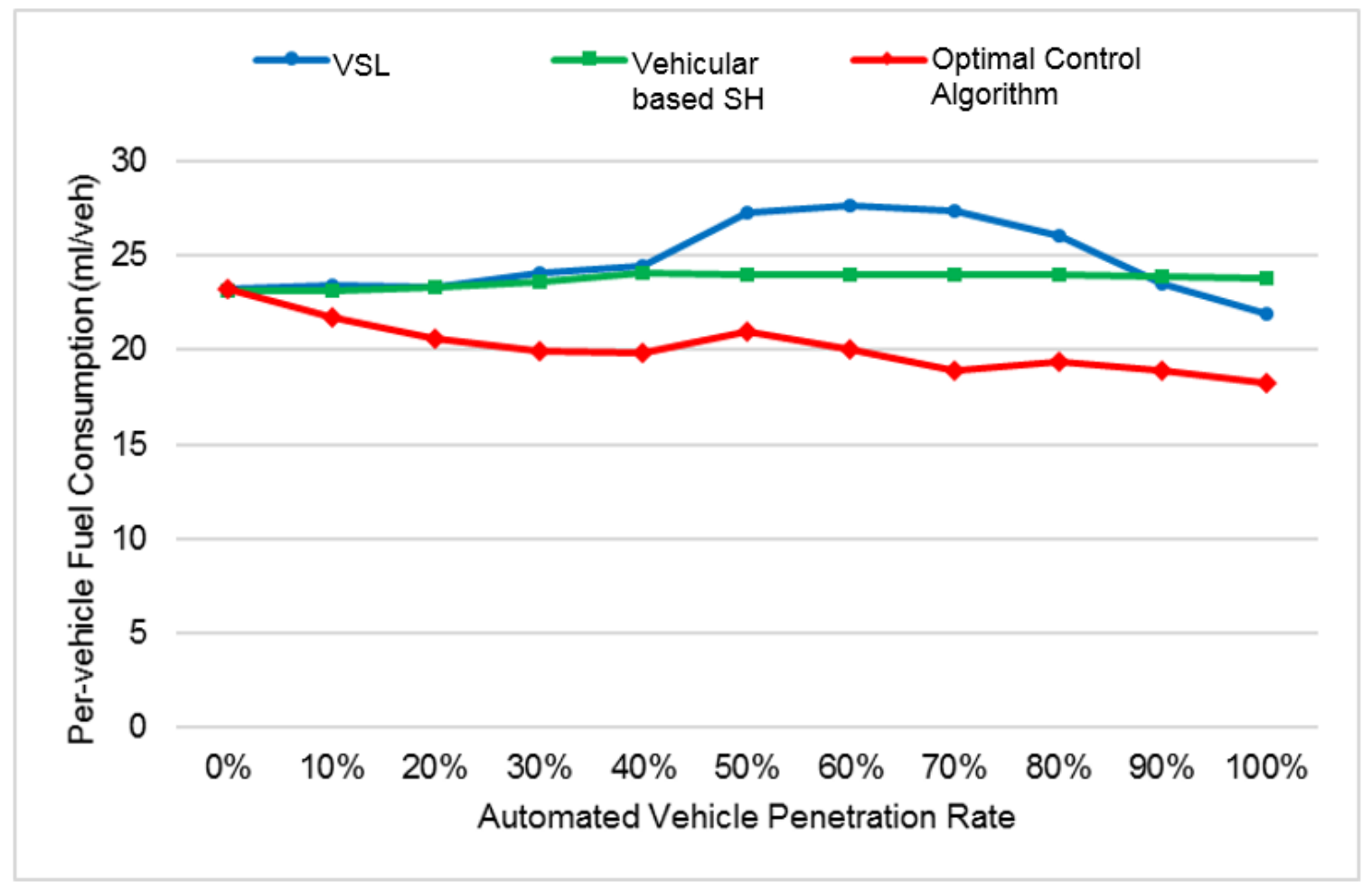

Figure 13. Fuel consumption under varying penetrations of CAVs

The results of ANOVA on the fuel consumption is summarized in Table 4. The single factor ANOVA results showed that each factor of the control algorithm and the CAV market penetration level impose significant impacts in the fuel consumptions at 0.05 
significant level. The F-value of 114 (p-value $<0.01$ ) indicated that the optimal control algorithm resulted smaller amounts of fuel consumptions compared to that of the VSL algorithm and the Veh-based SPD-HARM. Likewise, the factor of CAV market penetrations yielded the F-value of 3.9 ( $\mathrm{p}$-value $<0.001$ ), indicating that the CAV market penetrations levels imposed statistically significant impacts on the fuel consumptions.

The interaction influence of those two factors were shown to be significant on the fuel consumptions, by yielding the F-values of 4.4 (p-values $<0.001$ ). This also gives similar inference with the implication of the mobility measures - the improvement in the fuel consumption varied depending on which control algorithm is applied, i.e., the fuel reduction is greater under the optimal control algorithm than under the VSL algorithm or vehicle-based SPD-HARM algorithms.

\section{Table 4 ANOVA Results for Fuel Consumptions}

\begin{tabular}{|l|c|c|c|c|c|}
\hline \multicolumn{1}{|c|}{ Source } & $\begin{array}{c}\text { Sum of } \\
\text { Squares }\end{array}$ & df & $\begin{array}{c}\text { Mean } \\
\text { Square }\end{array}$ & F & Significance \\
\hline (A) Control Algorithm & 783.324 & 2 & 391.662 & 114.231 & 0.00 \\
\hline (B) CAV Market Penetration & 133.912 & 10 & 13.3912 & 3.90564 & 0.00 \\
\hline (A) $*$ B) & 301.831 & 20 & 15.0916 & 4.40156 & 0.00 \\
\hline Error & 1018.32 & 297 & 3.42869 & - & - \\
\hline Total & 2237.39 & 329 & - & - & - \\
\hline
\end{tabular}




\subsection{TRAFFIC STABILIZATION STRATEGY}

This section discusses the results of average speed, speed variances, and fuel consumptions of the base case of human drivers and the two approaches such as the existing ACC algorithm developed by Milane and Shladover (2014) and the proposed optimal speed control algorithm. As stated in Chapter 6.5, traffic volume is not considered as a factor to assess sensitivity of the control algorithms as the traffic stabilization strategy is the matter of improving car-following strategy of individual vehicle, which is opposed to the SPDHARM strategy that is to address a traffic bottleneck of which the characteristics heavily rely on the traffic volumes.

The result analysis for the traffic flow stabilization control includes the sensitivity analysis on the varying market penetrations of AVs and the investigation on the individual vehicle's speed profile. Summary of the results is represented in Table 5. 
Table 5. Comparisons of MOEs between ACC and Optimal Control Algorithm for Varying Market Penetrations of AVs

\begin{tabular}{|c|c|c|c|c|c|c|}
\hline \multirow[b]{2}{*}{$\begin{array}{c}\text { Vehicle } \\
\text { Compositions }\end{array}$} & \multicolumn{6}{|c|}{ MOE } \\
\hline & \multicolumn{2}{|c|}{$\begin{array}{l}\text { Average Speed } \\
\quad(\mathrm{mph})\end{array}$} & \multicolumn{2}{|c|}{$\begin{array}{c}\text { Acceleration } \\
\text { Variations } \\
\left(\mathrm{ft} / \mathrm{sec}^{2} / \mathrm{veh}\right)\end{array}$} & \multicolumn{2}{|c|}{$\begin{array}{l}\text { Fuel Consumptions } \\
\text { (ml/veh) }\end{array}$} \\
\hline $\begin{array}{l}\text { 100\% Human } \\
\text { Driven Vehicles }\end{array}$ & \multicolumn{2}{|c|}{32.80} & \multicolumn{2}{|c|}{7.86} & \multicolumn{2}{|c|}{67505.96} \\
\hline $\begin{array}{c}\text { Market } \\
\text { Penetrations of } \\
\text { Automated } \\
\text { Vehicles }\end{array}$ & $\begin{array}{c}\text { ACC } \\
\text { algorithm }\end{array}$ & $\begin{array}{c}\text { Optimal } \\
\text { Control } \\
\text { Algorithm }\end{array}$ & $\begin{array}{c}\text { ACC } \\
\text { algorithm }\end{array}$ & $\begin{array}{l}\text { Optimal } \\
\text { Control } \\
\text { Algorithm }\end{array}$ & $\begin{array}{c}\text { ACC } \\
\text { algorithm }\end{array}$ & $\begin{array}{l}\text { Optimal } \\
\text { Control } \\
\text { Algorithm }\end{array}$ \\
\hline $5 \%-2$ nd row & 32.95 & 33.22 & 9.79 & 9.47 & 63,296 & 62,718 \\
\hline $5 \%-10$ th row & 33.33 & 33.39 & 10.30 & 9.65 & 67,071 & 62,236 \\
\hline $10 \%$ & 33.15 & 33.24 & 11.62 & 11.99 & 59,178 & 58,864 \\
\hline $20 \%$ & 33.71 & 33.42 & 14.42 & 11.67 & 57,712 & 55,605 \\
\hline $30 \%$ & 33.47 & 33.46 & 15.49 & 12.83 & 57,571 & 55,723 \\
\hline $40 \%$ & 33.60 & 33.51 & 14.96 & 14.87 & 58,919 & 52,642 \\
\hline $50 \%$ & 33.92 & 33.59 & 15.04 & 12.62 & 58,542 & 54,095 \\
\hline $60 \%$ & 33.91 & 33.64 & 15.24 & 11.17 & 58,832 & 51,753 \\
\hline $70 \%$ & 34.00 & 33.62 & 14.75 & 11.23 & 58,327 & 51,663 \\
\hline $80 \%$ & 33.94 & 33.64 & 14.79 & 10.03 & 57,942 & 50,106 \\
\hline $90 \%$ & 34.01 & 33.24 & 14.63 & 6.98 & 58,262 & 47,946 \\
\hline $100 \%$ & 34.25 & 33.02 & 14.88 & 6.46 & 63,654 & 46,810 \\
\hline
\end{tabular}

\subsubsection{Under the perfect market penetration of $A V s$}

To begin with, the speed trajectories of the vehicles for the existing ACC algorithm and the proposed algorithm are explored along with the baseline scenario of $100 \%$ of 
human driven vehicles. The speed variations profiles are plotted by the lane that each vehicle belongs to at the current time. The discontinuity of the line represents the lane change maneuvers. The traffic passes through the control zone of $30 \mathrm{mph}, 45 \mathrm{mph}$, and then back to $30 \mathrm{mph}$ in an order.

In Figure 14, it is shortly after the vehicle speed achieves the level of speed limit, it is observed that the speed profile shows fluctuations although the vehicles are spaced at the desired headway distance with the desired speed level at the beginning. This is due to the instability of the free flow traffic that is cause when the vehicle density is near or exceeds the critical density (usually known as $k_{o}$, the traffic density at the maximum flow rate of the roadway) which is coupled with the effect of enhancement of fluctuation along with the collective effects of vehicles. 

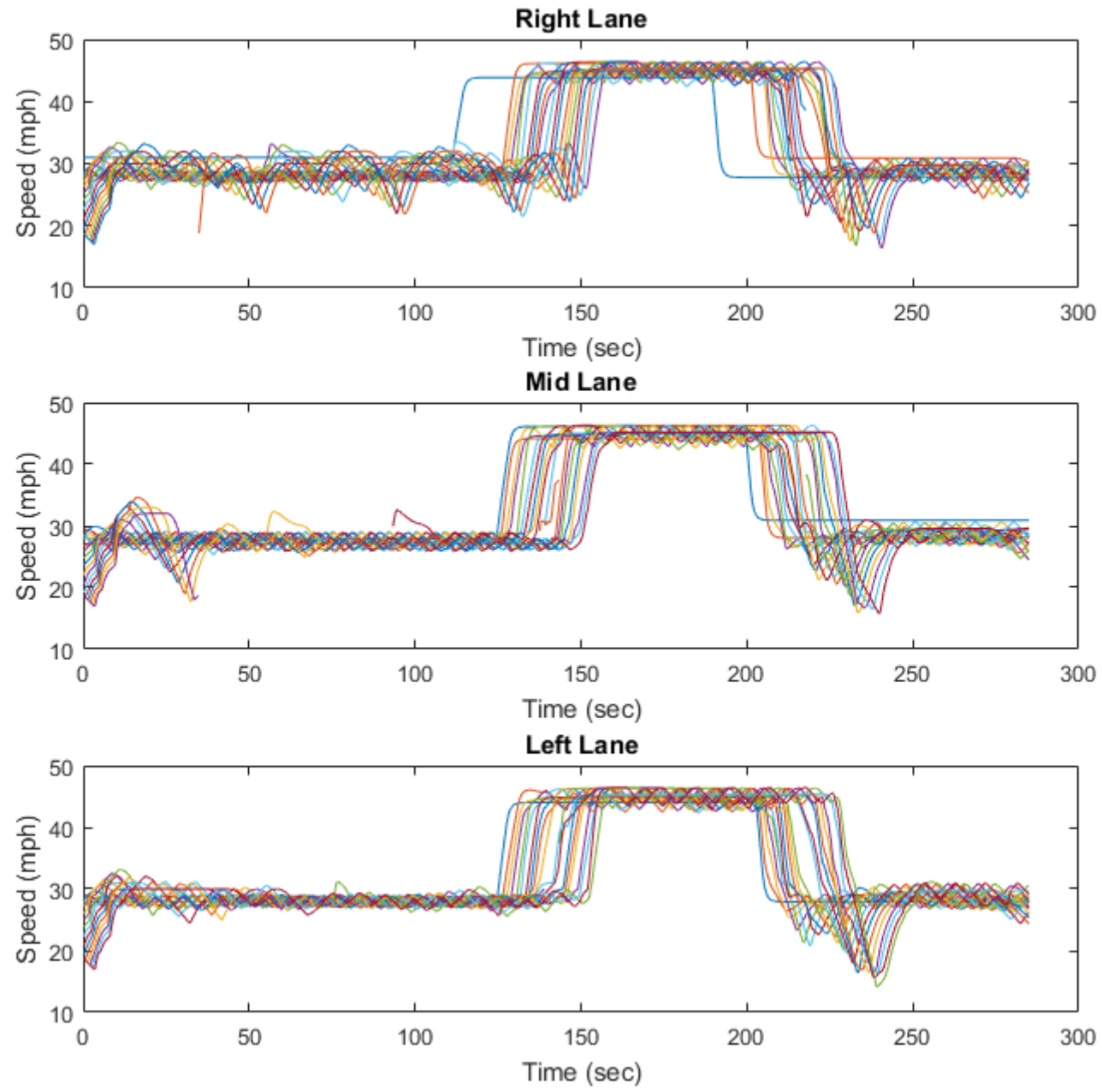

Figure 14. Speed trajectories of the case of $100 \%$ human driven vehicles.

When the control speed is back to $30 \mathrm{mph}$ after the $45-\mathrm{mph}$ zone, vehicles experiences significant speed drops until they recover its speed back to the speed limit due to the shock wave propagating backward incurring the fluctuation amplified. Such phenomena of concerns on fuel consumptions and traffic stabilizations. 

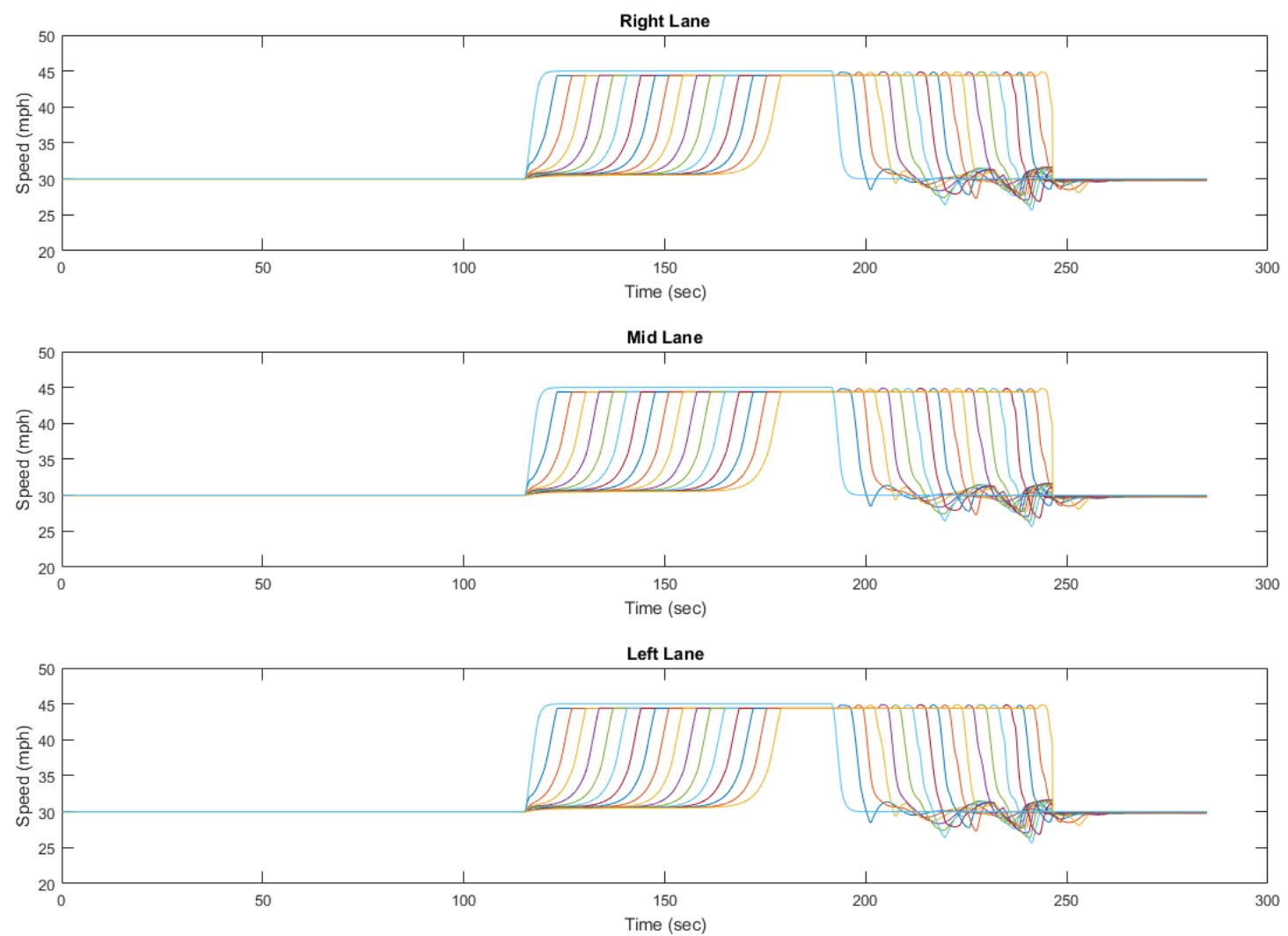

Figure 15. Speed trajectories of the $100 \%$ automated vehicles controlled by ACC

\section{algorithm.}

Figure 15 shows the vehicle speed profiles of the existing ACC algorithm that is implemented at the perfect market penetrations of automated vehicles. For a fair comparison, the ACC algorithm is slightly modified to constrain the maximum control speed as the speed limit.

Obvious benefit is to completely resolve the speed fluctuations within the first section of speed limit of $30 \mathrm{mph}$. The ACC algorithm controlled the vehicle to maintain its speed and distance error terms to be minimal upon the leverage of automated vehicles that does not associates perception reaction time. 
As the first leading vehicle drives through the roadway sections with different speed limits at the smooth speed transitions (the blue line located the front in Figure 15), the following vehicles show the exponential increases and decreases in speeds. Such behaviors are resulted as the ACC algorithm is aiming at maintaining the constant time headway from the preceding vehicle while not considering so-called optimal time headway that the subject vehicle should pursue. Such control approach is limited in resolving the speed fluctuation on the area where the speed limit reduces from $45 \mathrm{mph}$ to $30 \mathrm{mph}$, since the control approach is not capable to provide smooth transition especially when a vehicle encounter abrupt speed reduction and the fluctuating effect is still enhanced for the following vehicles. 

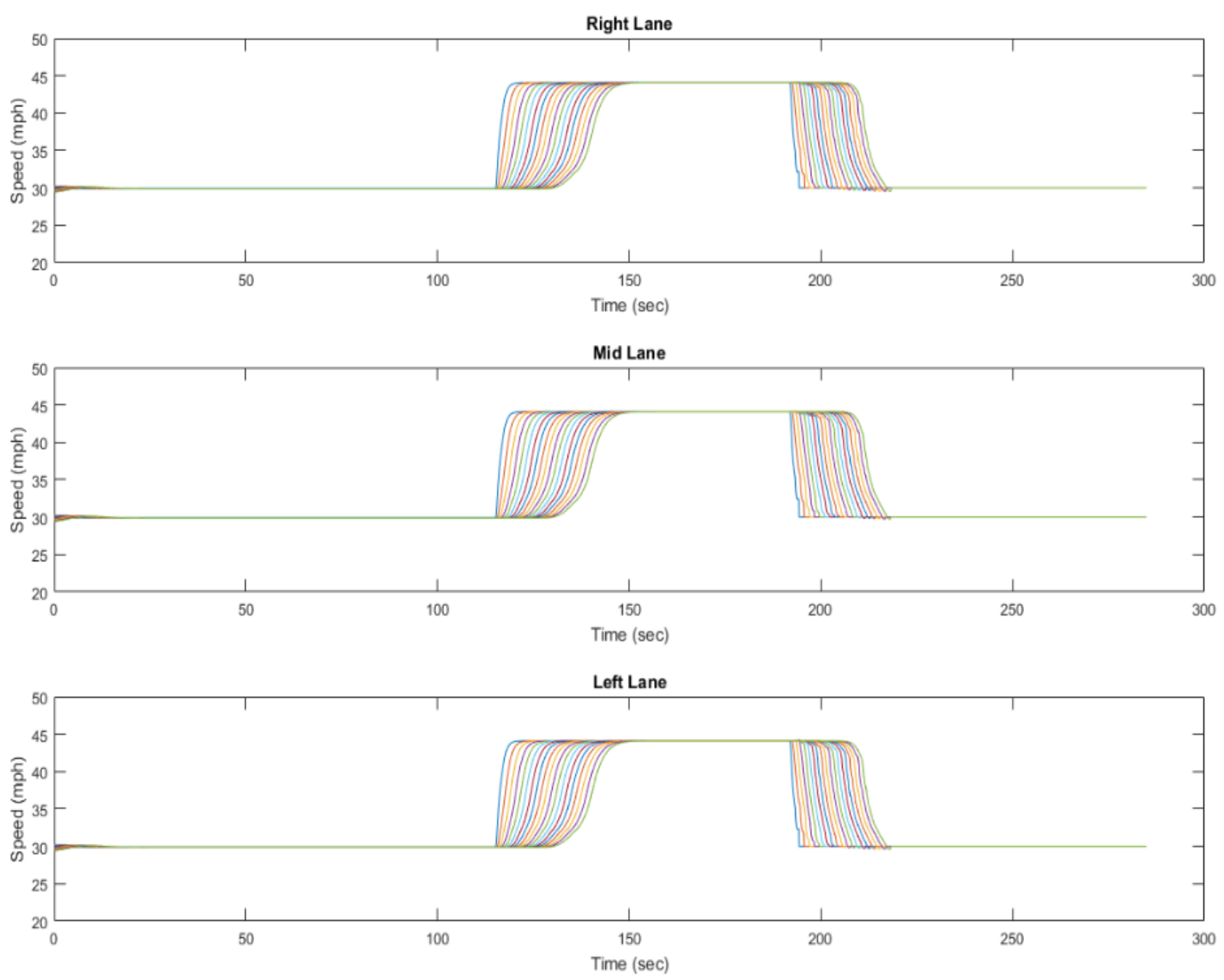

Figure 16. Speed trajectories of the case of $100 \%$ automated vehicles controlled by Optimal Control Algorithm.

Figure 16 shows the vehicle speed profiles of the optimal control algorithm of traffic stabilization strategy that is implemented at the perfect market penetration of automated vehicles. Likewise, the optimal control algorithm completely mitigated the speed fluctuations within the first section with the speed limit of $30 \mathrm{mph}$ by providing control to maintain the constant time headway from the preceding vehicle and with the leverage of the automated vehicles.

However, unlike the existing ACC algorithm, the speed profile represents sigmoid shape to increase and decrease to and from the speed limit while the vehicles react to the preceding vehicles' speed variations through the changes in speed limits, which is the most 
likely to minimize the acceleration variations. In addition, it is noted that the vehicles start their speed transition earlier than the case of existing ACC algorithm, which allows smoother transitions for the traffic platoon. This relies on how the control acceleration is determined; the existing ACC algorithm determines the acceleration policy by the equation considering the speed and error terms in a combined polynomial expression, while the optimal control algorithm finds the optimal trajectories under the speed and distance constrains separately, which is more explicit and can results more appropriate reaction to the preceding vehicle's trajectories in real-time.

\subsubsection{Under mixed traffic of $A V_{S}$ and $H D V_{S}$}

To evaluate the traffic flow stabilization control, the average speed, acceleration variations and the fuel consumptions are evaluated for varying market penetration rates of automated vehicles with the human driven vehicles.

\section{Mobility}

In Figure 17, the average speeds during the entire travel by different market penetrations are plotted for both the existing ACC algorithm and the optimal control algorithm. In general, the average speed variations does not significantly change by the penetrations of AVs controlled by either control strategies, and not significantly different between the two algorithms until the AV market penetrations of $90 \%$.

Under the market penetration of $100 \%$, the optimal control algorithm resulted the average speed slightly decrease to the same level with the speed of the baseline case of human drivers. This reminds the idea that the trajectory that optimizes acceleration 
variations does not necessarily benefit faster traffic flow. In a while, the slight increase in average speed for the existing ACC algorithm is explained by the exponential change patterns in speed during the speed transitions.

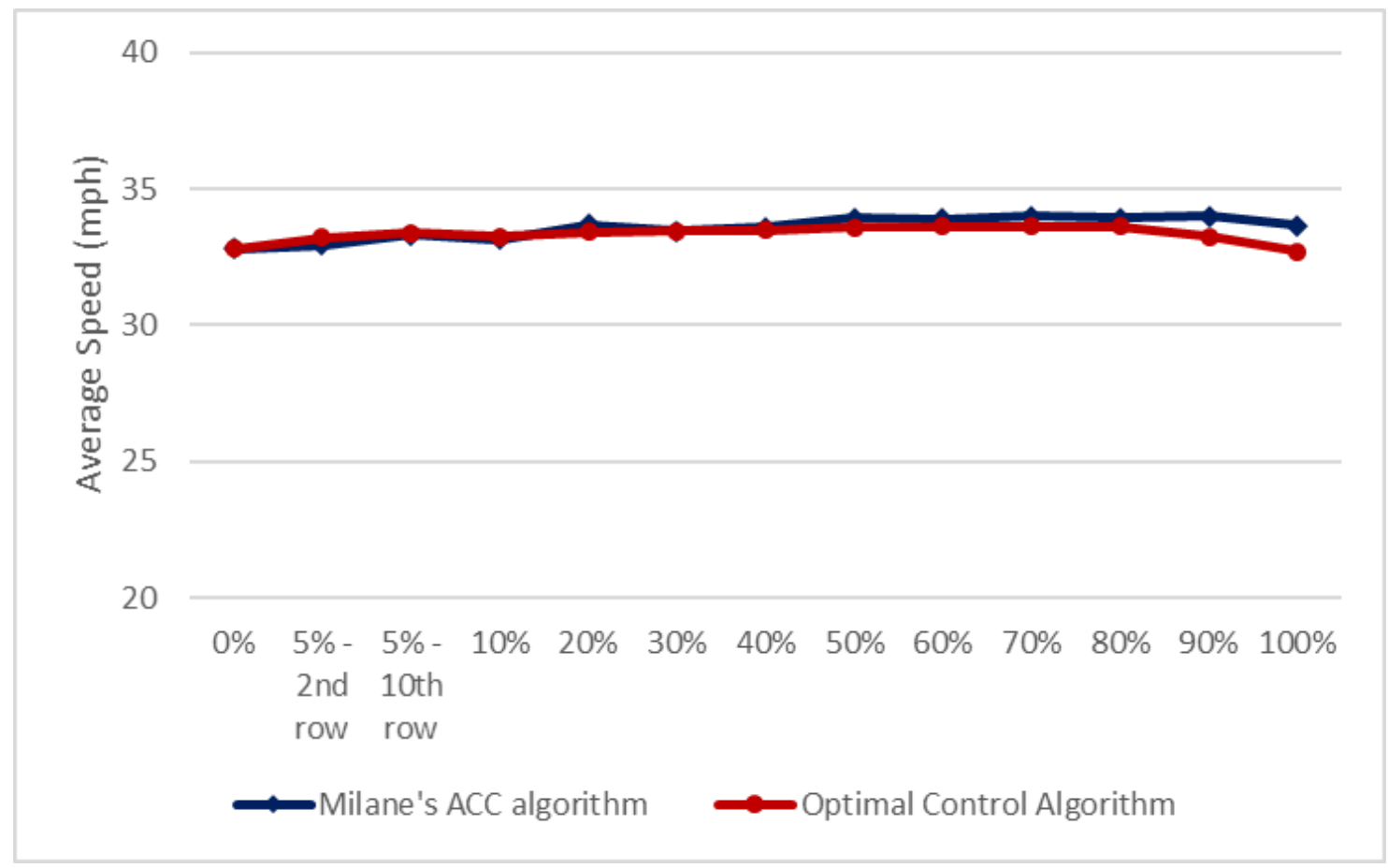

Figure 17. Average speed of ACC vs. Optimal Control Algorithm for varying market penetrations.

Table 6 ANOVA Analysis Results for Average Speed

\begin{tabular}{|l|c|c|c|c|r|}
\hline \multicolumn{1}{|c|}{ Source } & $\begin{array}{c}\text { Sum of } \\
\text { Squares }\end{array}$ & df & $\begin{array}{c}\text { Mean } \\
\text { Square }\end{array}$ & F & Significance \\
\hline (A) Control Algorithm & 1.72 & 1 & 1.72 & 14.64 & 0.00 \\
\hline $\begin{array}{l}\text { (B) AV Market } \\
\text { Penetration }\end{array}$ & 11.99 & 12 & 0.99 & 8.49 & 0.00 \\
\hline$(\mathrm{A}) *(\mathrm{~B})$ & 3.53 & 12 & 0.29 & 2.49 & 0.01 \\
\hline Error & 12.24 & 104 & 0.11 & - & - \\
\hline Total & 29.48 & 129 & - & - & - \\
\hline
\end{tabular}

To investigate the statistical significance of the individual impact of each factor such as the control algorithms and the AV market penetration level as well as the 
interaction impact of those two factors, the ANOVA analysis is conducted. As shown in Table 6, the impacts of each main factor on the average speed were significant at the 0.05 significance level. That is, the average speed under the optimal control algorithm is significantly different from the existing ACC algorithm. In addition, the different levels of AV market penetrations generated significant impacts on the average speed, i.e., the average speed increased with the higher level of AV penetrations between ACC algorithm and optimal control algorithm.

The influence of the interaction of the two factors were shown to be significant for the average speed, by yielding the F-values of 2.49 ( $p$-value $<0.025$ ). This results infers that the impacts of AV market penetrations on the average speed is different depending on the control algorithms. The improvements in average speed are greater under the optimal control algorithm than under the ACC algorithm.

\section{Acceleration Variations}

In Figure 18, the overall pattern of the acceleration variations for both of ACC and the optimal control algorithm show that the intrusion of any type of control impose adverse impacts in general. Upon the observations on the speed trajectories under the mixed traffic, it is seen that the human driven vehicles perform more lane changes as they follow AVs that are governed by distinctive speed patterns during the transitions of speed. Figure 19 and Figure 20 illustrates the speed trajectories under the AV market penetration of $40 \%$ for the ACC algorithm and the optimal control algorithm, respectively, where the high accelerations is identified. There are seen frequent lane change committed by the human 
drivers that follow the automated vehicles and thus created speed fluctuations for the consecutively following vehicles platoon.

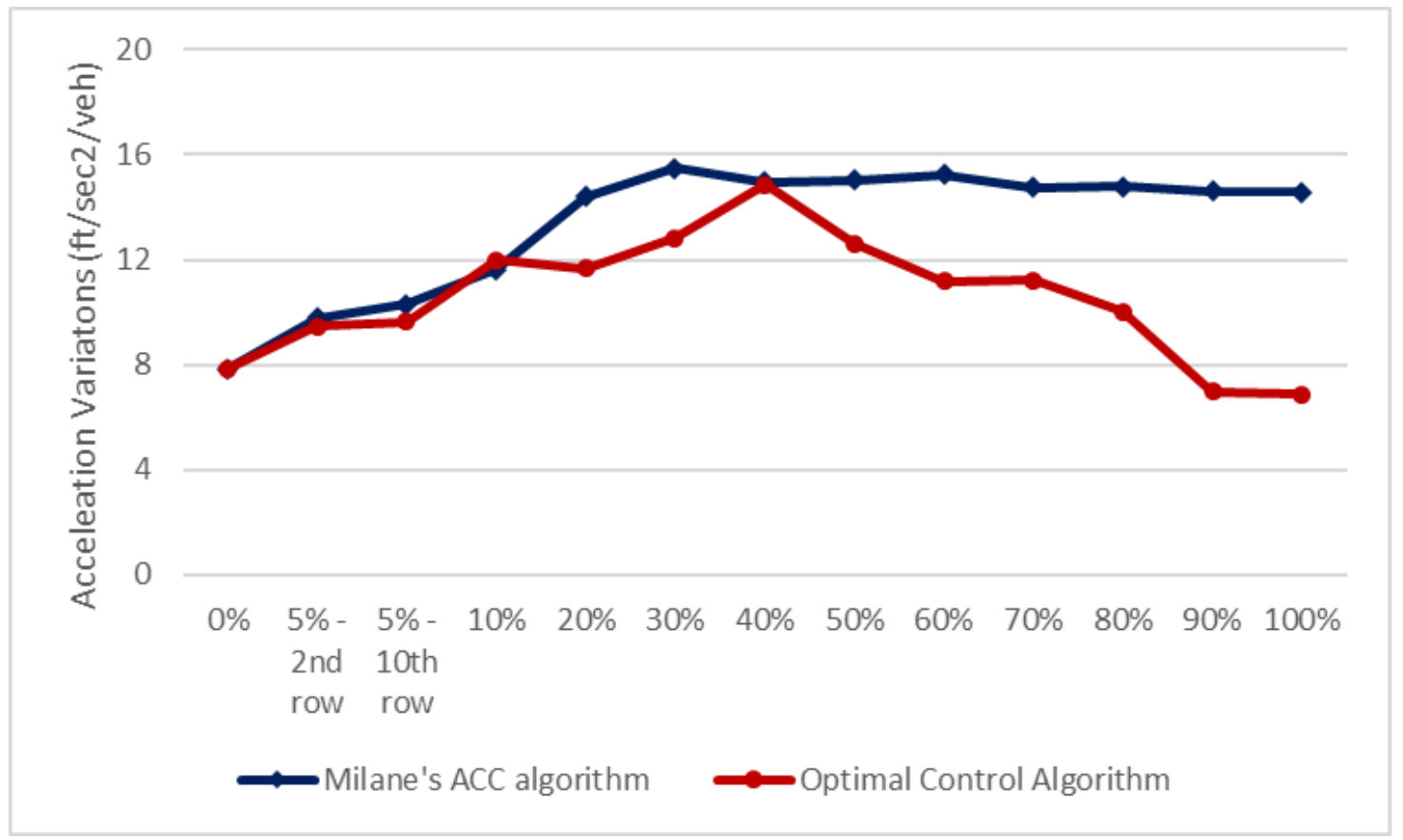

Figure 18. Per-vehicle acceleration variations of ACC vs. Optimal Control Algorithm for varying market penetrations of automated vehicles.

Unlike the ACC algorithm which shows continuously higher acceleration variations compared to the baseline case, the optimal control algorithm ends up reduced its acceleration variations at the AV market penetrations of $90 \%$.

It has to be mentioned that this results is only tested under specific case where the desired speed limits are changes. The existing ACC algorithm selected in this study is inherently designed for the purpose of reducing accelerations variations under typical carfollowing situation. Upon the scenario used in this research where the vehicles are required to change speed limit, when the preceding vehicle start speeding up as entering the new control zone, the following vehicle is meant to increase at higher speed to reduce its error 
term in speed and distance, which end up resulted in exponential increase and decrease patterns in speed.

On the contrary, the optimal control algorithm is able to serve as a pace-car while following the speeding vehicle, as the algorithm considers optimal headway time as a constraint while minimizing the acceleration variations under the explicit consideration of location and speed of preceding vehicles.
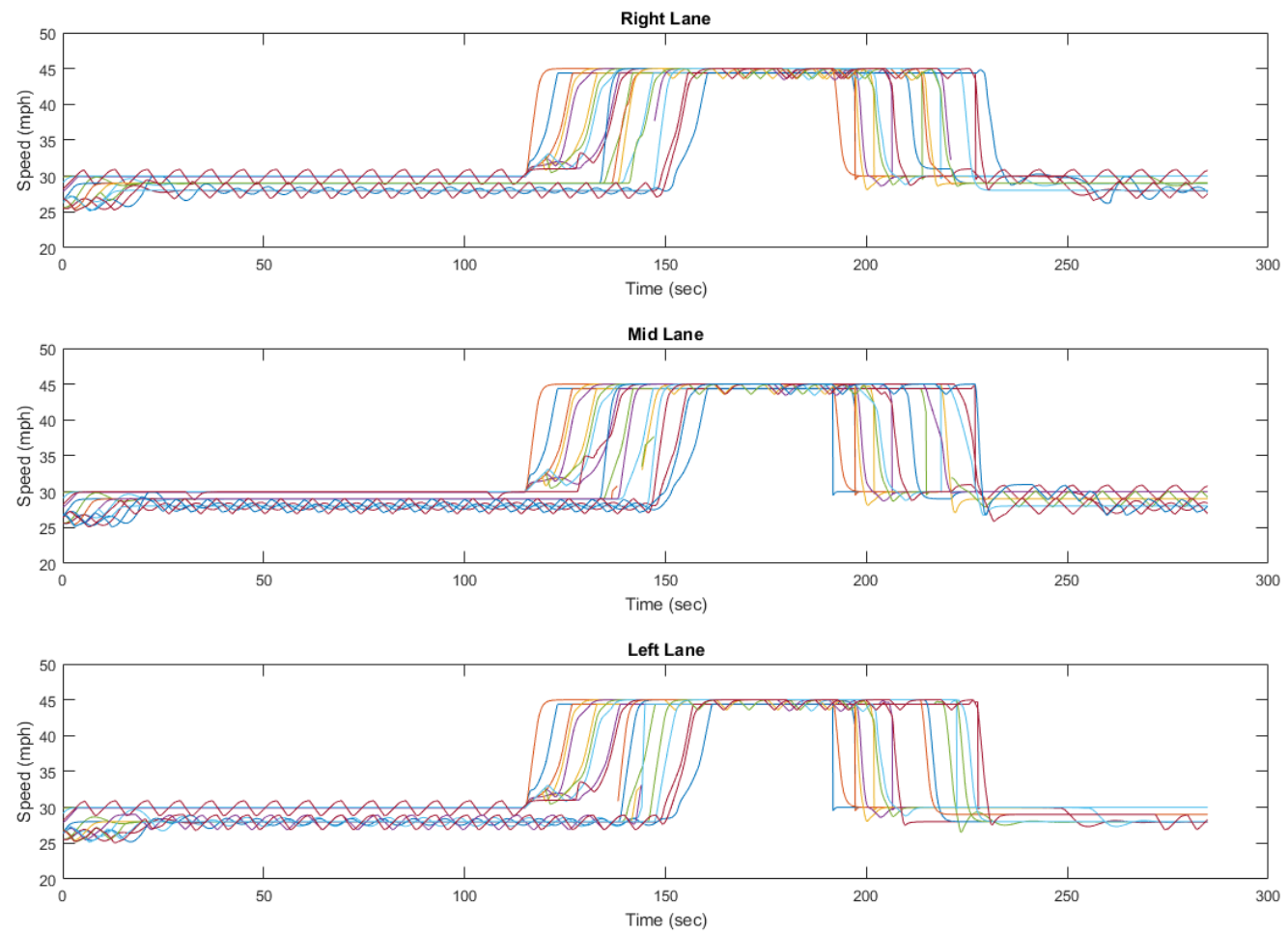

Figure 19 Speed trajectory of ACC algorithm under $40 \%$ of AV. 

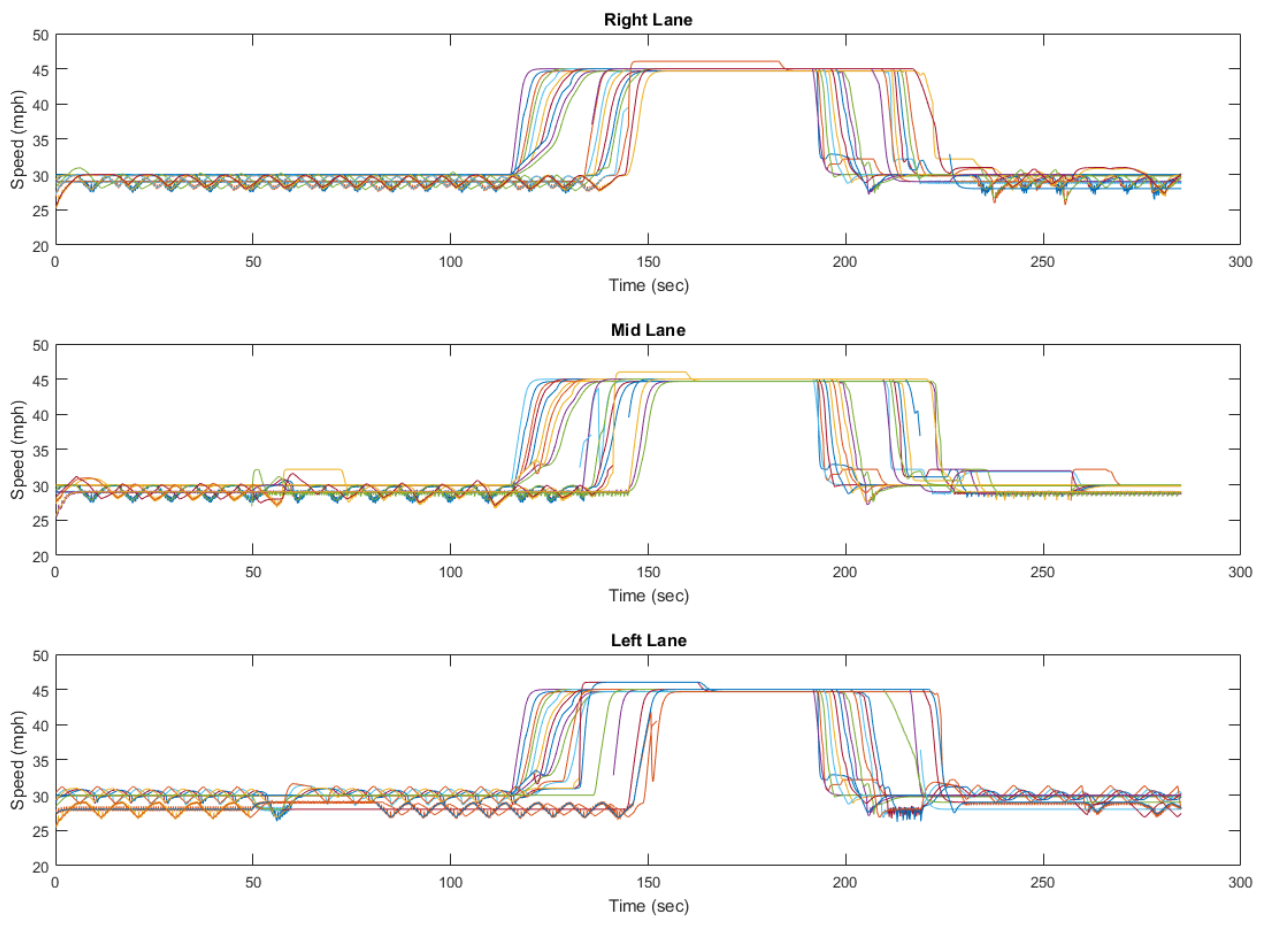

Figure 20 Speed trajectory of the optimal control algorithm under $40 \%$ of AV

The ANOVA analysis is conducted to investigate the significance of the comparisons results on the acceleration variations. As shown in Table 6, the impacts of the main factor on the acceleration variation is significant at the 0.05 significance level. That is, the acceleration variations under the optimal control algorithm is significantly lower than that of the ACC algorithm. In addition, the different levels of AV market penetrations generated significant impacts on the acceleration variations, i.e., the acceleration variation decreased with the higher level of AV penetrations between ACC algorithm and optimal control algorithm.

The influence of the interaction of the two factors were shown to be significant for the acceleration variations, by yielding the F-values of 2.77 (p-value $<0.001$ ). This results infers that the impacts of AV market penetrations on the acceleration variations is different 
depending on the control algorithms. The improvements in acceleration variation are greater under the optimal control algorithm than under the ACC algorithm.

Table 7 ANOVA Analysis Results for Acceleration Variations

\begin{tabular}{|l|c|c|c|c|r|}
\hline \multicolumn{1}{|c|}{ Source } & $\begin{array}{c}\text { Sum of } \\
\text { Squares }\end{array}$ & df & Mean Square & F & Significance \\
\hline $\begin{array}{l}\text { (A) Control } \\
\text { Algorithm }\end{array}$ & 252.43 & 1 & 252.43 & 37.58 & 0.00 \\
\hline $\begin{array}{l}\text { (B) AV Market } \\
\text { Penetration }\end{array}$ & 500.84 & 12 & 41.73 & 6.21 & 0.00 \\
\hline (A) * (B) & 223.75 & 12 & 18.64 & 2.77 & 0.00 \\
\hline Error & 698.49 & 104 & 6.71 & - & - \\
\hline Total & 1675.51 & 129 & - & - & - \\
\hline
\end{tabular}

\section{Fuel Consumptions}

As shown in Figure 21, in general, the ACC algorithm and the optimal control algorithm reduced the fuel consumptions as the market penetrations of $\mathrm{AV}$ increases.

The performance on the fuel consumptions showed no statistical difference between the ACC algorithm and the optimal control algorithm reaches up to the market penetrations rate of $30 \%$. The ACC algorithm leveled off at its fuel consumption improvements, whereas the optimal control algorithm continuously decreased the fuel consumptions and end up reduced by $18 \%$ over the human driven vehicle case and $57 \%$ over the existing ACC algorithm under the $100 \%$ of AV market penetrations. The ACC algorithm rather increases with its acceleration variations at the $100 \% \mathrm{AV}$ penetrations, which is incurred by the simultaneous changes in speed exponentially. 


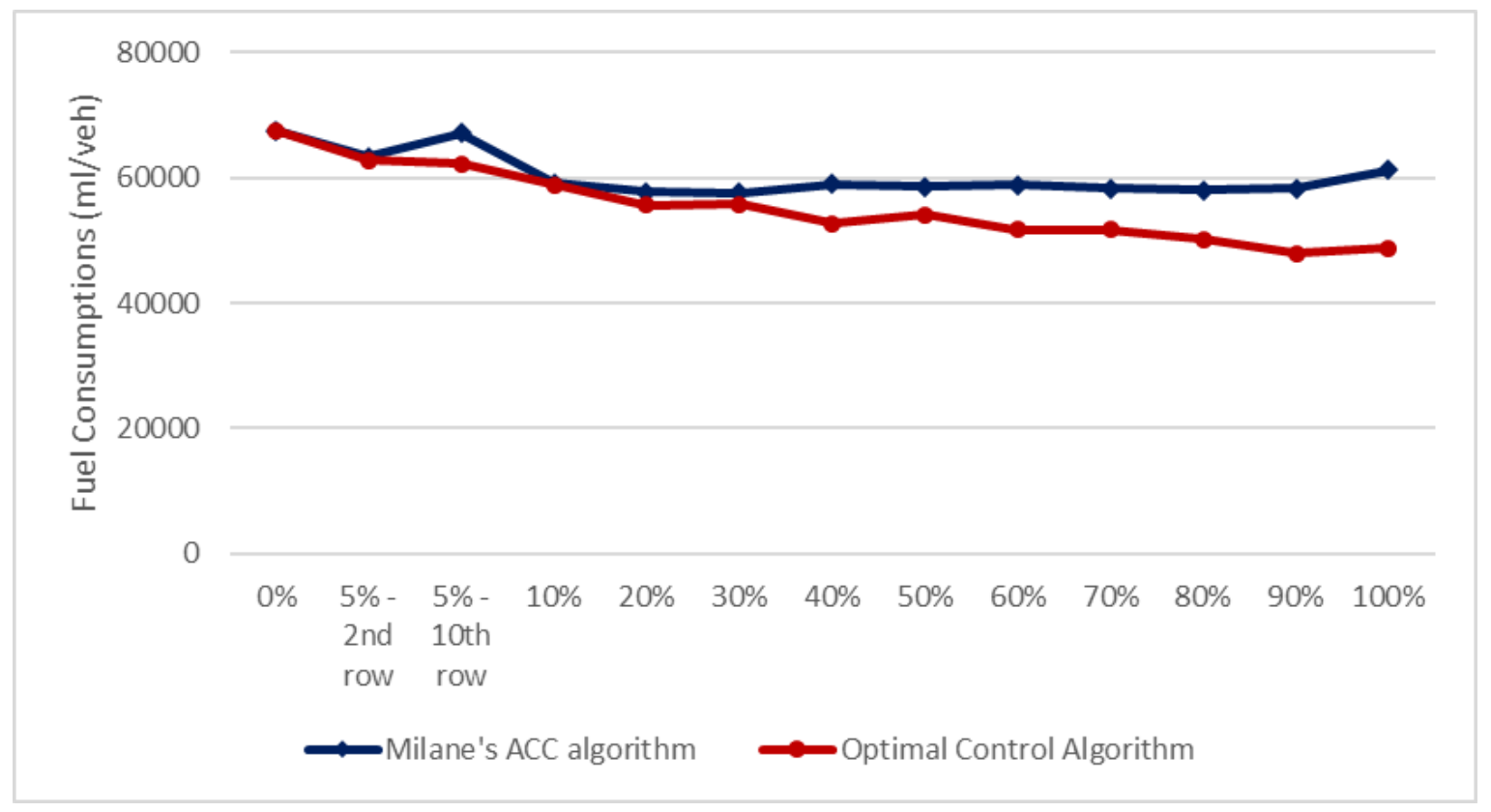

Figure 21. Fuel consumptions of ACC vs. Optimal Control Algorithm for varying market penetrations of automated vehicles.

The results of ANOVA on the fuel consumption is summarized in Table 8. The single factor ANOVA results showed that each factor of the control algorithm and the AV market penetration level impose significant impacts in the fuel consumptions at 0.05 significant level. The F-value of 42.27 ( $\mathrm{p}$-value $<0.01$ ) indicated that the optimal control algorithm resulted smaller amounts of fuel consumptions compared to that of the existing ACC algorithm. Likewise, the factor of AV market penetrations yields the F-value of 10.33 (p-value $<0.01$ ), which indicates that the AV market penetrations levels impose statistically significant impacts on the fuel consumptions.

The interaction influence of those two factors are shown not significant on the fuel consumptions, by yielding the F-values of 2.03 ( $\mathrm{p}$-values $>0.025$ ). This indicates that the 
fuel consumption variation pattern according to varied AV market penetrations is not affected either it is under optimal control algorithm or ACC algorithm.

Table 8 ANOVA Analysis Results for Fuel Consumptions

\begin{tabular}{|l|c|c|c|c|r|}
\hline \multicolumn{1}{|c|}{ Source } & $\begin{array}{c}\text { Sum of } \\
\text { Squares }\end{array}$ & df & $\begin{array}{c}\text { Mean } \\
\text { Square }\end{array}$ & F & Significance \\
\hline $\begin{array}{l}\text { (A) Control } \\
\text { Algorithm }\end{array}$ & $8.07 \times 10^{8}$ & 1 & $8.07 \times 10^{8}$ & 42.27 & 0.00 \\
\hline $\begin{array}{l}\text { (B) AV Market } \\
\text { Penetration }\end{array}$ & $2.36 \times 10^{9}$ & 12 & $1.97 \times 10^{8}$ & 10.33 & 0.00 \\
\hline (A) $($ B) & $4.65 \times 10^{8}$ & 12 & $3.88 \times 10^{7}$ & 2.03 & 0.03 \\
\hline Error & $1.98 \times 10^{9}$ & 104 & $1.90 \times 10^{7}$ & - & - \\
\hline Total & $5.62 \times 10^{9}$ & 129 & - & - & - \\
\hline
\end{tabular}

In summary of the evaluation results of traffic flow stabilization control, the existing state-of-the-art ACC algorithm demonstrated that the traffic flow stability is not fully achievable for multiple consecutive vehicles especially when the speed limit changes. Although the speed changes smoothly, the control policy includes a large enough instant jerk of speeds during the speed changes, which causes the unstable following trajectory of the following vehicles. Such instability on the existing ACC algorithm is solved by the optimal control strategy that is equipped with the logic considering the optimal headway to be maintained while the preceding vehicle's measurement is explicitly considered. 


\section{CHAPTER 8: CONCLUSIONS AND FUTURE RESEARCH}

\subsection{SUMMARY AND CONTRIBUTIONS}

The state-of-the-art traffic operations strategies adhere to heuristic approach which uses numerical method to find approximate solutions that are close to the true solutions within a certain range. This is because of the complexity of representing traffic dynamics coupled with human drivers' behaviors. Obviously, such heuristic approach does not guarantee system optimality and is often not implemented in the field due to their computational burden and the need of calibration efforts entailed to the technique of the algorithm. Various traffic control algorithms have been consistently evolved in a way to improve computational efficiency to realize real-time operations.

Although there have been consistent improvements in effectiveness, the research to develop control strategy attaining true optimality is still lacking. Furthermore, it is still a research need to optimize individual trajectory while considers vehicle platoon system.

This dissertation proposed an analytical approach-based control strategies using Pontryagin's Minimum Principle with an objective of minimization of control efforts (i.e., minimizing acceleration variations). The key merits of the proposed optimal control algorithm are:

- It guarantees true optimal strategies;

- It is computationally efficient for real-time operations; and

- It optimizes not only individual vehicle's longitudinal dynamics but also guarantees the optimality in terms of the vehicle platoon. 
In particular, two problems are addressed in this dissertation: (i) optimal control on the speed of the automated vehicles before they enter a speed reduction zone on a freeway; and (ii) optimal control on the speed of the automated vehicles to follow the preceding vehicle. The control problem is formulated and solved using Hamiltonian analysis to provide an analytical, closed-form solution that can be implemented in real time. The solution yields the optimal acceleration/deceleration of each vehicle under the hard safety constraint of rear-end collision avoidance.

The developed algorithms are implemented and evaluated using the advanced microscopic simulation tool that is developed in this research. A comprehensive set of scenarios is tested to evaluate the performance in various aspects. The factors considered include traffic volumes and the market penetrations of automated vehicles. To evaluate the performance of the proposed algorithm, existing state-of-the-art algorithms that are comparable to the proposed algorithm are modeled and tested under the controlled conditions.

The optimal control algorithm shows significant improvements in mobility, fuel consumption, and traffic flow stabilization compared to those of the base case and the stateof-the-art algorithms under varying market penetrations of automated vehicles.

The key findings on the speed harmonization strategies are:

- Under $100 \%$ of CAV market penetrations, the optimal control algorithm improves travel time and throughput for all traffic volumes over the baseline scenario, VSL, and the vehicular-based SPD-HARM algorithm. In particular, travel time is improved by $26-30 \%$ over the baseline scenario, by 
3-19\% over the VSL algorithm, and by $31-39 \%$ over the vehicular-based SPD-HARM algorithm for the three traffic volume cases.

- Under $100 \%$ of CAVs, the optimal speed control algorithm reduces fuel consumption of each vehicle by $19-22 \%$ over the baseline scenario, by $12-$ $17 \%$ over the VSL algorithm, and by $18-34 \%$ over the vehicular-based SPD-HARM algorithm for the three traffic volume cases (i.e., 10\% lower than the capacity, at the capacity, $10 \%$ great than the capacity).

- Under the mixed traffic of CAVs and HDVs, the optimal control algorithm constantly reduces the travel time as the market penetration rate increases, while the existing control algorithms rather degenerated mobility measures (travel time and vehicle throughput) under the CAV penetration near 50\%, and it requires at least $70 \%$ of market penetration to outperform the base case. Even under the low penetrations of CAVs, the adjacent following human driven vehicle not necessarily make sudden decelerations, but closely follow the preceding CAV to have the significant improvement.

- Under the mixed traffic of CAVs and HDVs, likewise the mobility evaluations, the per-vehicle fuel consumption with the optimal control algorithm is significantly reduced from that of the base case throughout the varying market penetrations of CAVs, while the existing VSL and the vehicular-based SPD-HARM algorithms results that the fuel consumption even increased with as the market penetrations in the range of 50-70\%. 
The key findings on the traffic flow stabilization strategies are:

- Under the $100 \%$ of market penetrations, the existing ACC algorithm and the optimal control algorithm resolve the speed fluctuations within the first section of speed limit of $30 \mathrm{mph}$. As the vehicles drive through the roadway sections with different speed limit, the optimal control algorithm results the speed trajectories to be the sigmoid shape when the vehicles increase and decrease to and from the earlier speed limit, which is the most likely to minimize the acceleration variations. On the contrary, the existing ACC algorithm results exponential shapes of speeds to increase and decrease to and from the speed limit. It is because the existing ACC algorithm, as similar as the other existing ACC algorithms, determines the acceleration policy by the equation considering the speed and error terms in a combined polynomial expression. The optimal control algorithm finds the optimal trajectories under the speed and distance constrains separately, which is more explicit and can results more appropriate reaction to the preceding vehicle's trajectories in real-time.

- Under mixed traffic of CAVs and HDVs, for both of ACC algorithm and the optimal control algorithm, the acceleration variations become worse with even small market penetrations of CAVs by incurring additional lane changes by the human driven vehicles following AVs with distinctive speed patterns during the transitions of speed limit. However, unlike the ACC algorithm which shows continuously higher acceleration variations 
compared to the baseline case, the optimal control algorithm end up reducing its acceleration variations at the $\mathrm{AV}$ market penetrations of $90 \%$.

- As for fuel consumptions, both the existing ACC algorithm and the optimal control algorithm reduced the fuel consumptions compared to the base case of human driven vehicles as the market penetrations of AV increases. Until the market penetrations rate reaches to $30 \%$, the performance on the fuel consumptions are statistically the same between the ACC algorithm and the optimal control algorithm. The optimal control algorithm continuously decreased the fuel consumptions and end up reduced by $18 \%$ over the human driven vehicle case and 57\% over the existing ACC algorithm under the $100 \%$ of AV market penetrations.

The experimental results in this dissertation demonstrates the feasibility of the control algorithm under mixed traffic of automated vehicles and human driven vehicles and provides quantitative assessment in various aspects of mobility, fuel economy and traffic flow stability compared to the existing practices. 


\subsection{FUTURE RESEARCH}

As a pioneering effort in developing an analytical approach to address traffic congestion problem, several assumptions are made to determine the scope of the research. The future research is to relax these assumptions and investigate the implications in the solution.

First, the proposed framework assumes full automation that does not involve human intervention at any case. According to the taxonomy specified by National Highway Traffic Safety Administration (NHTSA) (SEMA 2014), there are five levels of autonomy for automated vehicles: no automation (level 0), function-specific automation (level 1), combined function automation (level 2), limited self-driving automation (level 3), and full self-driving automation (level 4). These transition levels should not be overlooked when the implications on the algorithm are evaluated. Depending on which level of autonomy is considered, compliance rate on the control algorithm and human behavioral reaction against to the control policy will be a critical factor to determine the performance. To address this, a driving simulator that involves human drivers' input in a real time can be integrated to the various scenarios of different vehicle automations. The quantitative results on the measures of effectiveness and the qualitative evaluations through survey may be of use for evaluating the system.

Second, the lane changes of automate vehicles are not considered in this research. Knowing that lane change maneuver is one of the major reasons for creating a traffic bottleneck, integrating a proper lane change algorithm to the current control framework and investigate the implications on the multi-lane and the lane-drop situation is critical. For example, a CAV is likely to seek for a chance to commit a lane change when there is a lane 
drop ahead or it has significantly slower vehicles in front. By leveraging the communications between $\mathrm{CAVs}$ and central controller, the information such as willingness of committing lane change and the target location/lane are communicated between the CAVs and controller. The controller determines which CAVs that can commit lane change based on the traffic conditions factors (e.g., distance to the potential bottleneck, the traffic volumes in each lanes) at every update interval. The CAVs that are designated to do the lane change seeks for a speed trajectory to merge under the constraints of the location and the speed of the two adjacent vehicles in the target lane (i.e., the vehicle which after the subject CAV is going to follow, and the vehicle that the CAV will cut in front). Depending on which combination of those two adjacent vehicles are (whether human driven vehicles or CAVs), the control speed trajectory of a subject CAV should follow different strategies. The final aim for the lane change control is to maintain stability of the traffic flow while reducing the possibility of traffic breakdown due to the lane changes.

Finally, the assumption on the perfect information might overpromised the benefits compared to the actual benefit from the field deployment of the proposed framework. Although it is relatively straightforward to extend the results to the case that this assumption is relaxed, implicating the factors of information errors, delays, cyberattack in the control system process is worthy of attention. 


\section{REFERENCES}

Alessandri, A., Febbraro, A. Di, Ferrara, A., and Punta, E. (1998). "Optimal control of freeways via speed signalling and ramp metering." 6, 771-780.

Van Arem, B., Van Driel, C., and Visser, R. (2006). "The Impact of Cooperative Adatpitve Cruise Control on Traffic-Flow Characteristics." Intelligent Transportation Systems, IEEE Transactions on, 7.4, 429-436.

Brinckerhoff, P., Farradyne, T., and Burgess, J. C. (2008). Active Traffic Management Concept of Operations. Washington State Department of Transportation (WSDOT).

Carlson, R., Papamichail, I., Papageorgiou, M., and Messmer, A. (2010). “Optimal mainstream traffic flow control of large-scale motorway networks." Transportation Research Part C: Emerging Technologies, 18(2), 193-212.

Chen, D., and Ahn, S. (2015). "Variable speed limit control for severe non-recurrent freeway bottlenecks." Transportation Research Part C: Emerging Technologies.

Cheng, T., Lai, K., Venus Lun, Y., and Wong, C. W. (2013). “Green shipping management." Transportation Research Part E, 55, 1-2.

Dowling, R., Skabardonis, A., and Alexiadis, V. (2004). "Traffic Analysis Toolbox Volume III : Guidelines for Applying Traffic Microsimulation Modeling Software.” Rep. No. FHWA-HRT-04-040, U.S. DOT, Federal Highway Administration, Washington, D.C, III(July), 146.

Frejo, J. R. D., and Camacho, E. F. (2012). "Global Versus Local MPC Algorithms in Freeway Traffic Control With Ramp Metering and Variable Speed Limits." IEEE Transactions on Intelligent Transportation Systems, 13(4), 1556-1565.

Golestan, K., Soua, R., Karray, F., and Kamel, M. S. (2016). "Situation awareness within 
the context of connected cars: A comprehensive review and recent trends." Information Fusion.

Gouy, M., Diels, C., Stevens, A., Reed, N., and Burnett, G. (2013). "Do drivers reduce their headway to a lead vehicle because of the presence of platoons in traffic? A conformity study conducted within a simulator." IET Intelligent Transport Systems, 7(2), 230-235.

Hale, D. K., and Lu, X. (2016). "Introduction of Cooperative Vehicle-to- Infrastructure Systems to Improve.” (March).

Hegyi, A., Hoogendoorn, S. P., Schreuder, M., Stoelhorst, H., and Viti, F. (2008). "SPECIALIST: A dynamic speed limit control algorithm based on shock wave theory." Intelligent Transportation Systems, 2008. ITSC 2008. 11th International IEEE Conference on, IEEE, 827-832.

Hegyi, A., De Schutter, B., and Hellendoorn, H. (2005a). "Model predictive control for optimal coordination of ramp metering and variable speed limits." Transportation Research Part C: Emerging Technologies.

Hegyi, A., De Schutter, B., and Hellendoorn, H. (2005b). "Model predictive control for optimal coordination of ramp metering and variable speed limits." Transportation Research Part C: Emerging Technologies, 13(3), 185-209.

Hegyi, A., Schutter, B., and Hellendoorn, J. (2003). “Optimal coordination of variable speed limits to suppress shock waves." Transportation Research Record: Journal of the Transportation Research Board, (1852), 167-174.

Hung, S., Givigi, S. N., and Member, S. (2017). “A Q-Learning Approach to Flocking With UAVs in a Stochastic Environment." 47(1), 186-197. 
J. Qin, Zheng, W. X., and Gao, H. (2012). "Coordination of multiple agents with doubleintegrator dynamics under generalized interaction topologies." IEEE Trans. Syst., Man, Cybern. B, Cybern., 42(1), 44-57.

Jovanovi, A., Nikoli, M., and Teodorovi, D. (2017). "Area-wide urban traffic control: A Bee Colony Optimization approach.”

Juan, Z., Zhang, X., and Yao, H. (2004). "Simulation Research and Implemented Effect Analysis of Variable Speed Limits on Freeway." 894-898.

Kamal, M. A. S., Mukai, M., Murata, J., and Kawabe, T. (2011). "Ecological Vehicle Control on Roads With Up-Down Slopes." IEEE Transactions on Intelligent Transportation Systems, 12(3), 783-794.

Kesting, A., Treiber, M., Schönhof, M., and Helbing, D. (2007). “Adaptive cruise control design for active congestion avoidance."

Khondaker, B., and Kattan, L. (2015). "Variable speed limit: A microscopic analysis in a connected vehicle environment."

Kwon, E., Park, C., Lau, D., and Kary, B. (2011). “Minnesota Variable Speed Limit System: Adaptive Mitigation of Shock Waves for Safety and Efficiency of Traffic Flows." Washington DC.

Learn, S. (2016). "Speed Harmonization Using Connected and Automated Vehicles." University of Virginia.

Lee, J., Dailey, D. J., Bared, J. G., and Park, B. (2013a). “Simulation-based evaluations of real-time variable speed limit for freeway recurring traffic congestion." 92th Annual Meeting of Transportation Research Board.

Lee, J., Park, B., Malakorn, K., and So, J. (2013b). "Sustainability assessments of 
cooperative vehicle intersection control at an urban corridor." Transportation Research Part C: Emerging Technologies.

Liu, Y., and Passino, K. M. (2000). "Swarm Intelligence: Literature Overview."

Lu, X.-Y., Varaiya, P., Horowitz, R., Su, D., and Shladover, S. E. (2010). “A new approach for combined freeway variable speed limits and coordinated ramp metering." Intelligent Transportation Systems (ITSC), 2010 13th International IEEE Conference on, IEEE, 491-498.

Lu, X., Lee, J., Chen, D., Bared, J., Dailey, D., and Shladover, S. E. (2014). “Freeway Micro-simulation Calibration : Case Study Using Aimsun and VISSIM with Detailed Field Data." Transportation Research Board 93rd Annual Meeting.

Ma, J., Li, X., Shladover, S., Rakha, H. A., Lu, X.-Y., Jagannathan, R., and Dailey, D. J. (2016). "Freeway Speed Harmonization.” IEEE TRANSACTIONS ON INTELLIGENT VEHICLES, 1(1).

Milakis, D., Snelder, M., Van Arem, B., Van Wee, G. P., and Homem De Almeida Rodriguez Correia, G. (2016). "Scenarios about development and implications of automated vehicles in the Netherlands." 95th Annual Meeting Transportation Research Board, Washington, USA, 10-14 January 2016; Authors version, TRB.

Milanés, V., and Shladover, S. E. (2014). "Modeling cooperative and autonomous adaptive cruise control dynamic responses using experimental data." Transportation Research Part C: Emerging Technologies, 48, 285-300.

Montgomery. (2001). Introduction to Factorial Design; The Advantages of Factorials.

Olia, A., Abdelgawad, H., Abdulhai, B., and Razavi, S. N. (2015). "Traffic-Flow Characteristics of Cooperative vs. Autonomous Automated Vehicles." Washington 
DC.

Ozatay, E., Ozguner, U., Onori, S., and Rizzoni, G. (2012). "Analytical solution to the minimum fuel consumption optimization prob- lem with the existence of a traffic light." in Proc. of DSCC.

Papageorgiou, M., Kosmatopoulos, E., and Papamichail, I. (2008). "Effects of Variable Speed Limits on Motorway Traffic Flow.” Transportation Research Record: Journal of the Transportation Research Board, 2047, 37-48.

Papageorgiou, M., and Marinaki, M. (1995). A Feasible Direction Algorithm for the Numerical Solution of Optimal Control Problems. Dynamic Systems and Simulation Laboratory, Technical University of Crete, Chania, Greece.

Park, B., and Lee, J. (2009). “Assessing sustainability impacts of route guidance system under cooperative vehicle infrastructure environment.” 2009 IEEE International Symposium on Sustainable Systems and Technology, IEEE, 1-6.

Park, B., and Yadlapati, S. S. (2003). "Development and Testing of Variable Speed Limit Logics At Work Zones Using Simulation.” 2003, Washington, DC.

Pontryagin, L. S. (1987). L.S. Pontryagin: Mathematical Theory of Optimal Processes. CRC Press; English ed edition.

PTV. (2014). "PTV VISSIM 7 USER MANUAL."

Qiu, C., Shen, H., Sarker, A., Soundararaj, V., Devine, M., Rindos, A., and Ford, E. (2016). “Towards Green Transportation : Fast Vehicle Velocity Optimization for Fuel Efficiency." 59-66.

Rajamani, R., and Zhu, C. (2002). "Semi-autonomous Adaptive Cruise Control Systems." Vehicular Technology, IEEE Transactions, 51.5, 1186-1192. 
Rajasekhar, A., Lynn, N., Das, S., and Suganthan, P. N. (2016). "Computing with the collective intelligence of honey bees - A survey."

Rios-Torres, J., and Malikopoulos, A. (2016). "A Survey on the Coordination of Connected and Automated Vehicles at Intersections and Merging at Highway OnRamps." IEEE Transactions on Intelligent Transportation Systems.

Roberts, M. (2012). "I-70 pace cars experiment is working, CDOT says - just not all the time." $<$ http://www.westword.com/news/i-70-pace-cars-experiment-is-workingcdot-says-just-not-all-the-time-5880518> (May 23, 2016).

Robinson, M. (2000). “Examples of Variable Speed Limit Applications. Speed Management Workshop.” Transportation Research Board.

S. A. P. Quintero, Collins, G. E., and Hespanha, J. P. (2013). "Flocking with fixed-wing UAVs for distributed sensing: A stochastic optimal control approach." in Proc. Amer. Control Conf. (ACC), Washington, DC, USA, 2025-2031.

Seiler, P., Pant, A., and Hedrick, K. (2004). "Disturbance propagation in vehicle strings." IEEE Trans. Autom. Control, 49, 1835-1842.

SEMA (Specialty Equipment Market Association). (2014). "5 Levels of Vehicle Automation." <https://www.sema.org/sema-enews/2017/11/ettn-tech-alert-nhtsasae-define-5-levels-of-vehicle-automation>.

Shaw, E., and Hedrick, J. K. (2007). "String stability analysis for heterogeneous vehicle strings." Proc. American Control Conference, 3118-3125.

Shladover, S., Su, D., Lu, X. (2012). "Impacts of cooperative adaptive cruise control on freeway traffic flow.” Transportation Research Record, 2343, 63-70.

Stern, R. E., Cui, S., Laura, M., Monache, D., Bhadani, R., Bunting, M., Churchill, M., 
Hamilton, N., Haulcy, R. 'mani, Pohlmann, H., Wu, F., Piccoli, B., Seibold, B., Sprinkle, J., and Work, D. B. (2017). “Dissipation of stop-and-go waves via control of autonomous vehicles: Field experiments."

Sugiyama, Y., Fukui, M., Kikuchi, M., Hasebe, K., Nakayama, A., Nishinari, K., Tadaki, S. I., and Yukawa, S. (2008). "Traffic jams without bottlenecks-experimental evidence for the physical mechanism of the formation of a jam." New Journal of Physics, 10.

TRB. (2010). Highway Capacity Manual 2010. Washington DC.

VDOT. (2013). "VDOT GOVERNANCE DOCUMENT TRAFFIC OPERATIONS AND SAFETY ANALYSIS MANUAL (TOSAM) - Version 2.0.”

Wang, M., Daamen, W., Hoogendoorn, S. P., and van Arem, B. (2014). "Rolling horizon control framework for driver assistance systems. Part I: Mathematical formulation and non-cooperative systems." Transportation Research Part C: Emerging Technologies.

Welch, G., and Bishop, G. (2006). An Introduction to the Kalman Filter.

Zheng, Y., Jin, L., Gao, L., Li, K., Wang, Y., and Wang, F. (2016). “Development of a Distributed Cooperative Vehicles Control Algorithm Based on V2V Communication." Procedia Engineering. 


\section{Appendix}

\section{$\underline{\text { 1. Simulation Sample Size Evaluations }}$}

A. Speed Harmonization under various traffic volumes at $100 \%$ automated vehicles

\begin{tabular}{|c|r|r|r|r|r|r|r|r|r|}
\hline & \multicolumn{3}{|c|}{ Travel time } & \multicolumn{3}{c|}{ Veh Throughput } & \multicolumn{3}{c|}{ Per Vehicle FC } \\
\hline $\begin{array}{c}\text { Traffic } \\
\text { Volumes } \\
\text { (vph) }\end{array}$ & VSL & $\begin{array}{c}\text { Vehicle- } \\
\text { based } \\
\text { SH }\end{array}$ & $\begin{array}{c}\text { Optimal } \\
\text { control } \\
\text { algorithm }\end{array}$ & VSL & $\begin{array}{c}\text { Vehicle- } \\
\text { based } \\
\text { SH }\end{array}$ & $\begin{array}{c}\text { Optimal } \\
\text { control } \\
\text { algorithm }\end{array}$ & VSL & $\begin{array}{c}\text { Vehicle- } \\
\text { based } \\
\text { SH }\end{array}$ & $\begin{array}{c}\text { Optimal } \\
\text { control } \\
\text { algorithm }\end{array}$ \\
\hline 1620 & 1.33 & 1.43 & 2.14 & 0.00 & 0.00 & 0.00 & 1.63 & 0.86 & 1.80 \\
\hline 1800 & 2.35 & 2.35 & 2.54 & 0.00 & 0.00 & 0.00 & 1.52 & 0.42 & 2.56 \\
\hline 1980 & 1.83 & 2.23 & 2.08 & 0.01 & 0.01 & 0.00 & 0.82 & 0.88 & 2.39 \\
\hline
\end{tabular}

B. Speed Harmonization under various market penetrations of automated vehicles

\begin{tabular}{|c|c|c|c|c|c|c|c|c|c|}
\hline & \multicolumn{3}{|c|}{ Travel time } & \multicolumn{3}{|c|}{ Veh Throughput } & \multicolumn{3}{|c|}{ Per Vehicle FC } \\
\hline $\begin{array}{c}\text { Market } \\
\text { Penetrati } \\
\text { ons of } \\
\text { Automat } \\
\text { ed } \\
\text { Vehicles }\end{array}$ & VSL & $\begin{array}{c}\text { Vehicle } \\
\text {-based } \\
\text { SH }\end{array}$ & $\begin{array}{l}\text { Optimal } \\
\text { control } \\
\text { algorithm }\end{array}$ & VSL & $\begin{array}{c}\text { Vehicle- } \\
\text { based } \\
\text { SH }\end{array}$ & $\begin{array}{l}\text { Optima } \\
1 \\
\text { control } \\
\text { algorit } \\
\mathrm{hm}\end{array}$ & VSL & $\begin{array}{c}\text { Vehicle- } \\
\text { based } \\
\mathrm{SH}\end{array}$ & $\begin{array}{l}\text { Optimal } \\
\text { control } \\
\text { algorithm }\end{array}$ \\
\hline $0 \%$ & 0.14 & 1.11 & 2.89 & 0.00 & 0.00 & 0.01 & 0.10 & 0.09 & 1.04 \\
\hline $10 \%$ & 0.80 & 2.39 & 5.85 & 0.00 & 0.00 & 0.00 & 0.54 & 0.61 & 0.71 \\
\hline $20 \%$ & 1.10 & 2.76 & 7.23 & 0.00 & 0.00 & 0.00 & 0.94 & 1.00 & 3.84 \\
\hline $30 \%$ & 1.24 & 1.31 & 4.96 & 0.00 & 0.00 & 0.00 & 1.06 & 1.02 & 3.89 \\
\hline $40 \%$ & 1.38 & 2.93 & 5.32 & 0.00 & 0.01 & 0.01 & 3.00 & 1.29 & 4.67 \\
\hline $50 \%$ & 1.26 & 1.16 & 2.15 & 0.01 & 0.01 & 0.01 & 1.10 & 1.46 & 4.52 \\
\hline $60 \%$ & 1.17 & 1.06 & 2.17 & 0.00 & 0.01 & 0.01 & 2.19 & 1.03 & 4.23 \\
\hline $70 \%$ & 0.08 & 0.10 & 0.22 & 0.00 & 0.00 & 0.00 & 1.78 & 0.42 & 1.35 \\
\hline $80 \%$ & 1.42 & 1.04 & 2.56 & 0.00 & 0.00 & 0.00 & 2.41 & 0.47 & 1.77 \\
\hline $90 \%$ & 1.00 & 0.51 & 1.53 & 0.00 & 0.01 & 0.01 & 0.57 & 0.51 & 0.84 \\
\hline $100 \%$ & 2.35 & 2.35 & 2.54 & 0.00 & 0.00 & 0.00 & 1.52 & 0.42 & 2.56 \\
\hline
\end{tabular}


C. Traffic flow stabilization control under various market penetrations of automated vehicles

\begin{tabular}{|c|c|c|c|c|c|c|}
\hline \multirow[b]{2}{*}{ Vehicle Compositions } & \multicolumn{6}{|c|}{ MOE } \\
\hline & \multicolumn{2}{|c|}{$\begin{array}{l}\text { Average Speed } \\
\text { (mph) }\end{array}$} & \multicolumn{2}{|c|}{$\begin{array}{c}\text { Acceleration } \\
\text { Variations } \\
\left(\mathrm{ft} / \mathrm{sec}^{2} / \mathrm{veh}\right)\end{array}$} & \multicolumn{2}{|c|}{$\begin{array}{l}\text { Fuel Consumptions } \\
\text { (ml/veh) }\end{array}$} \\
\hline $\begin{array}{c}\text { 100\% Human Driven } \\
\text { Vehicles }\end{array}$ & \multicolumn{2}{|c|}{0.56} & \multicolumn{2}{|c|}{3.50} & \multicolumn{2}{|c|}{5.36} \\
\hline $\begin{array}{l}\text { Market Penetrations of } \\
\text { Automated Vehicles }\end{array}$ & $\begin{array}{c}\text { ACC } \\
\text { algorithm }\end{array}$ & $\begin{array}{l}\text { Optimal } \\
\text { Control } \\
\text { Algorithm }\end{array}$ & $\begin{array}{c}\text { ACC } \\
\text { algorithm }\end{array}$ & $\begin{array}{l}\text { Optimal } \\
\text { Control } \\
\text { Algorithm }\end{array}$ & $\begin{array}{c}\text { ACC } \\
\text { algorithm }\end{array}$ & $\begin{array}{c}\text { Optimal } \\
\text { Control } \\
\text { Algorithm }\end{array}$ \\
\hline $5 \%-2$ nd row & 0.11 & 0.02 & 1.16 & 1.01 & 0.50 & 3.56 \\
\hline $5 \%-10$ th row & 0.02 & 0.02 & 1.39 & 1.01 & 1.01 & 3.56 \\
\hline $10 \%$ & 0.02 & 0.02 & 1.70 & 1.62 & 0.67 & 1.88 \\
\hline $20 \%$ & 0.02 & 0.02 & 1.59 & 1.64 & 1.01 & 2.25 \\
\hline $30 \%$ & 0.02 & 0.02 & 1.04 & 1.24 & 1.24 & 2.02 \\
\hline $40 \%$ & 0.02 & 0.02 & 1.62 & 2.17 & 1.63 & 5.33 \\
\hline $50 \%$ & 0.02 & 0.02 & 1.48 & 1.50 & 1.98 & 3.46 \\
\hline $60 \%$ & 0.02 & 0.02 & 2.22 & 2.08 & 1.47 & 2.79 \\
\hline $70 \%$ & 0.02 & 0.02 & 1.59 & 1.21 & 1.28 & 2.25 \\
\hline $80 \%$ & 0.02 & 0.02 & 1.33 & 1.24 & 1.24 & 3.02 \\
\hline $90 \%$ & 0.02 & 0.02 & 0.03 & 0.01 & 0.07 & 0.56 \\
\hline $100 \%$ & 0.00 & 0.00 & 0.00 & 0.00 & 0.00 & 0.00 \\
\hline
\end{tabular}




\section{$\underline{\text { 2. Results of Traffic Flow Stabilization Control }}$}

A. $5 \%$ of AVs: three AVs located at the $2^{\text {nd }}$ row

\section{ACC Algorithm}
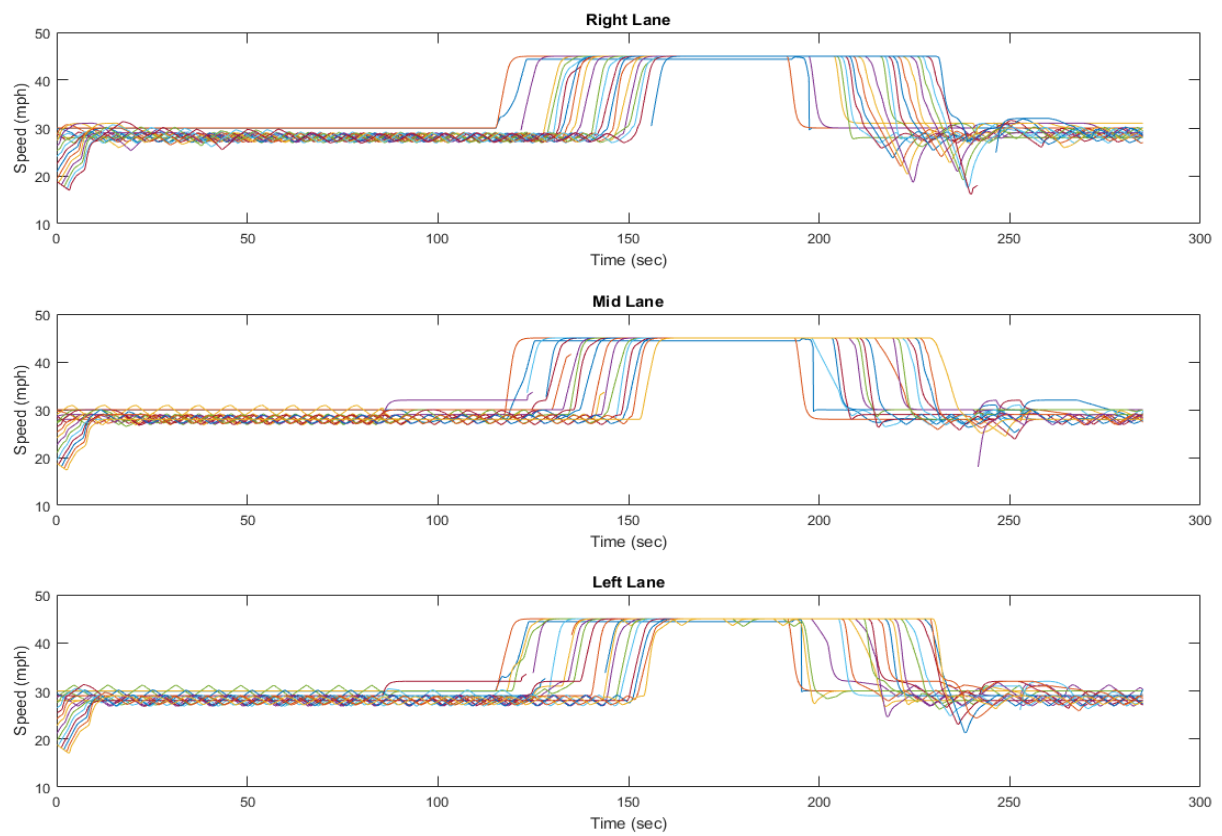

\section{Optimal Control Strategy}
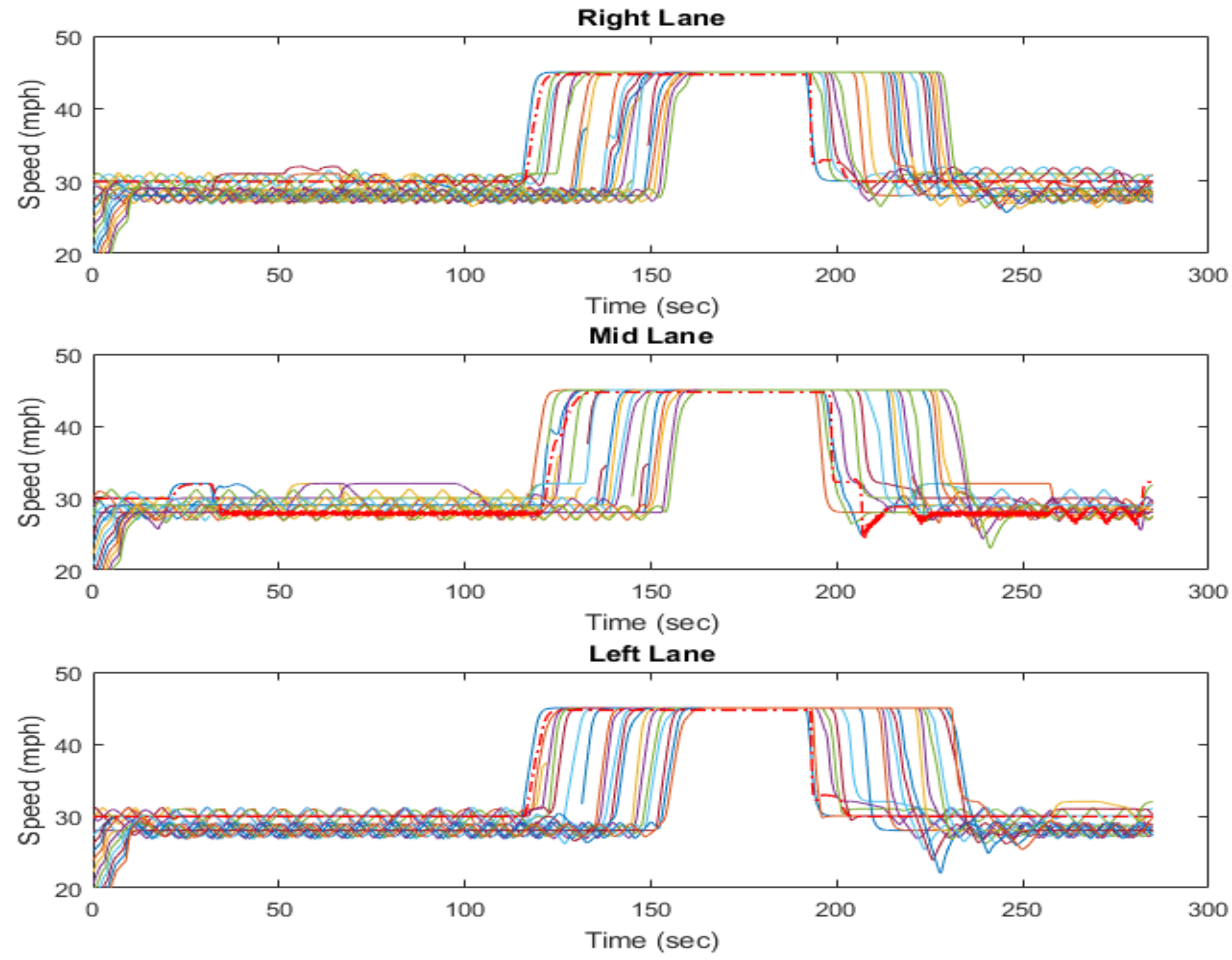
B. $5 \%$ of AVs: three AVs located at the $10^{\text {th }}$ row

\section{ACC Algorithm}
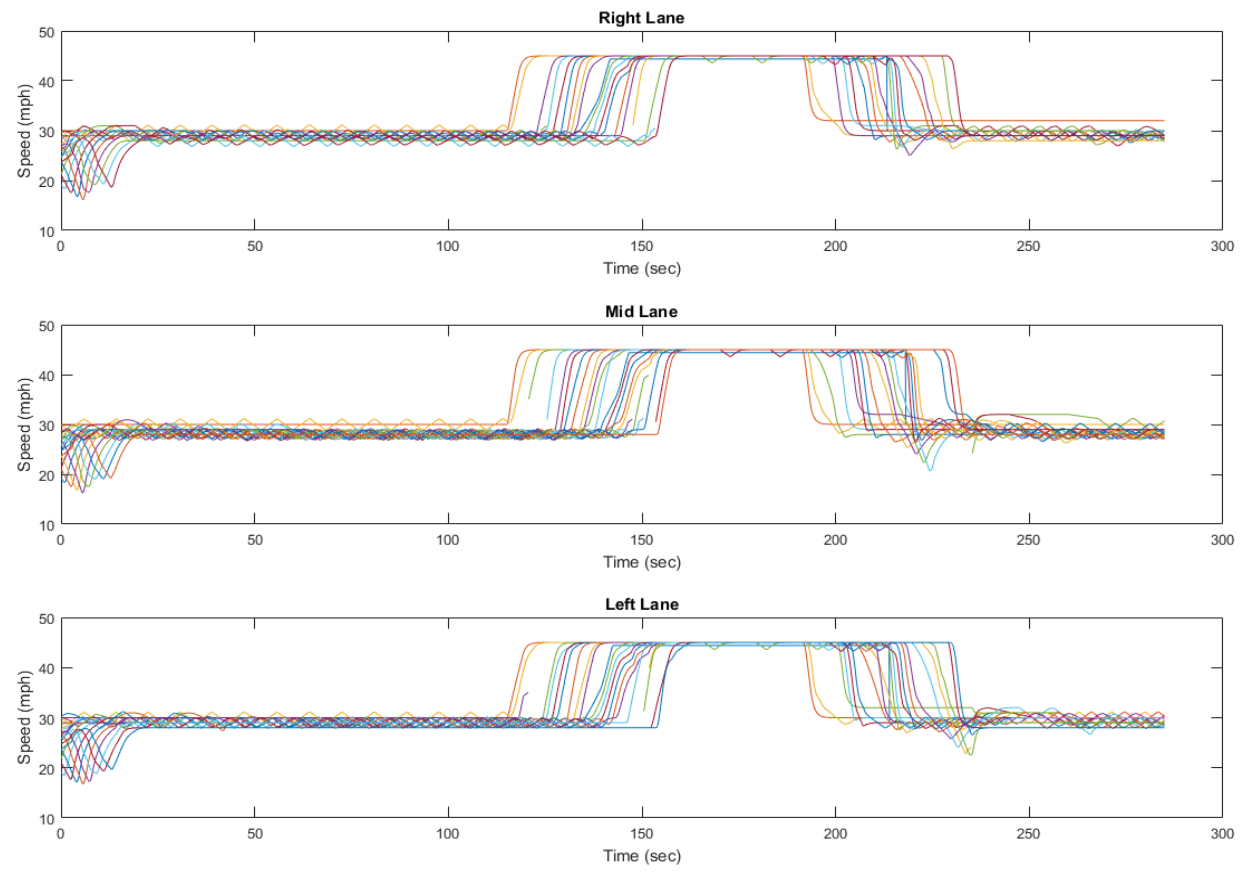

Optimal Control Algorithm
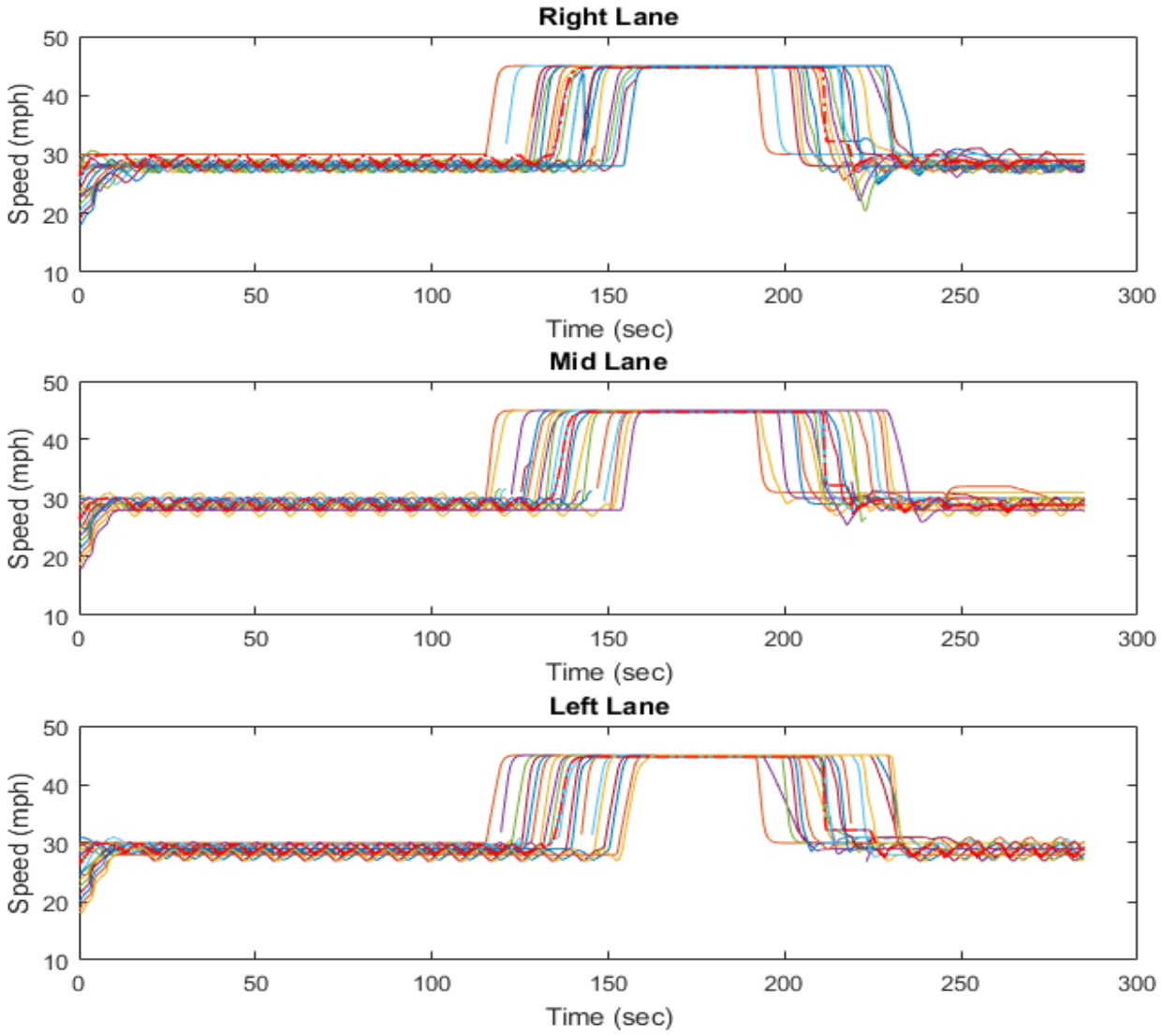


\section{C. $10 \%$ of AVs}

\section{ACC Algorithm}
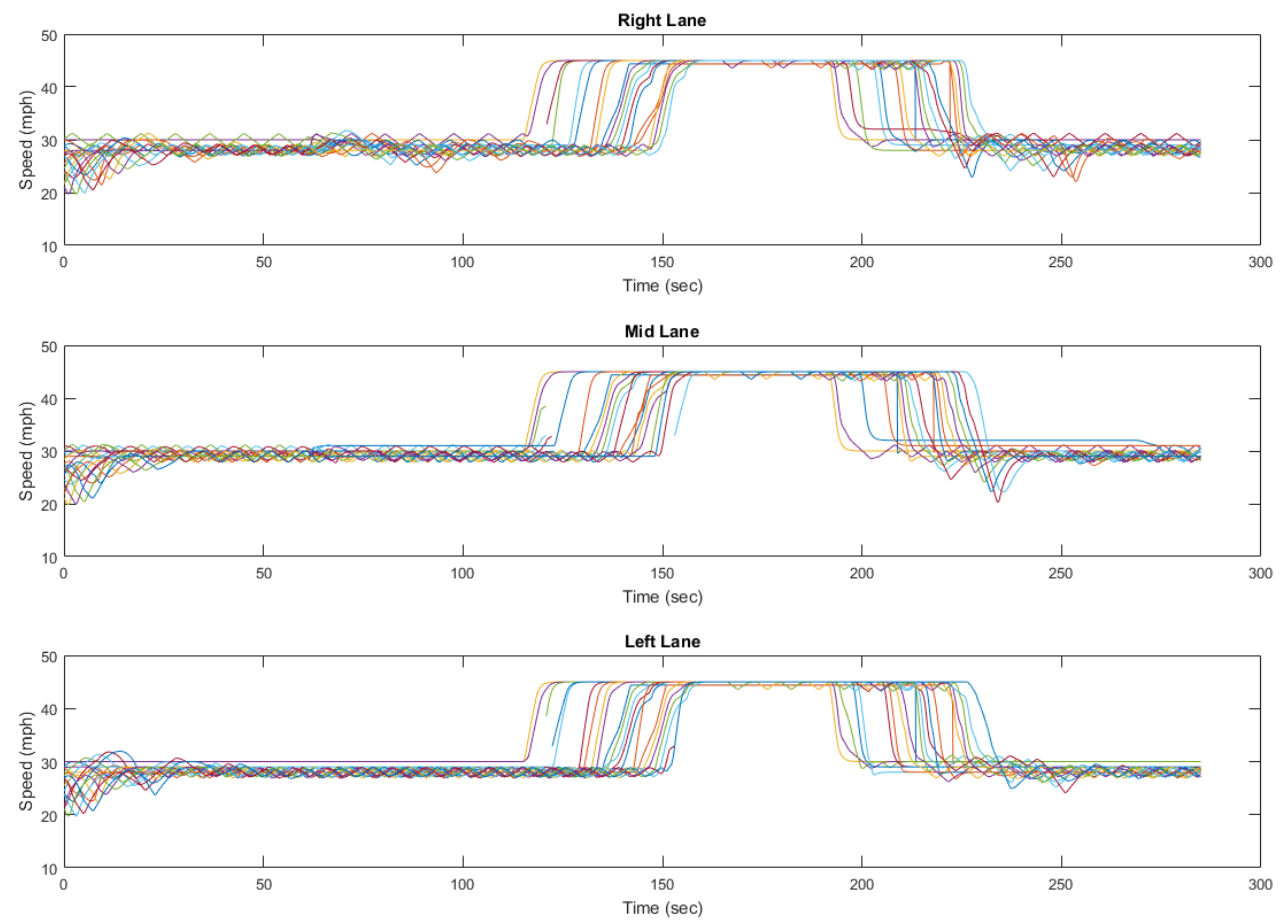

\section{Optimal Control Algorithm}
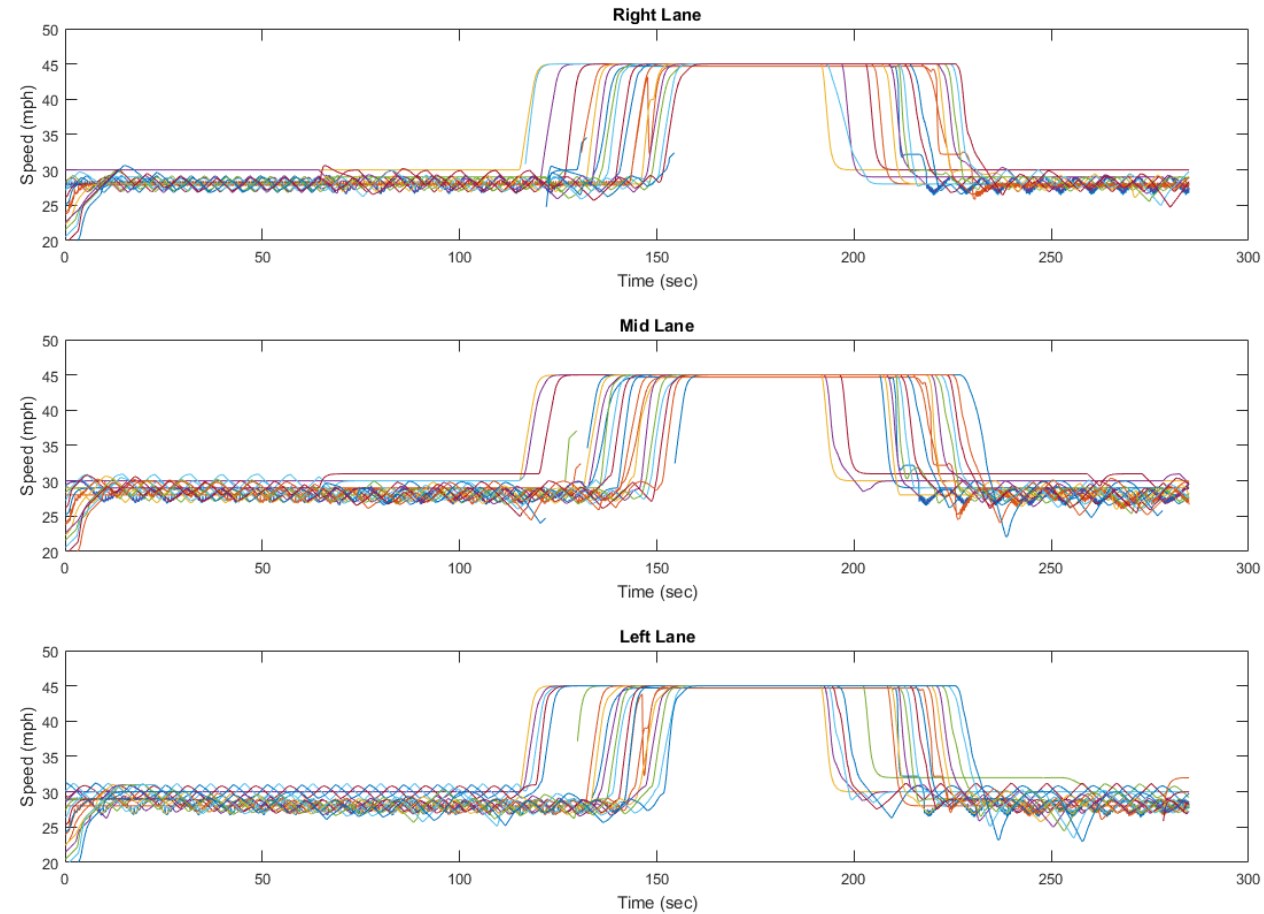


\section{D. $20 \%$ of AVs}

\section{ACC Algorithm}
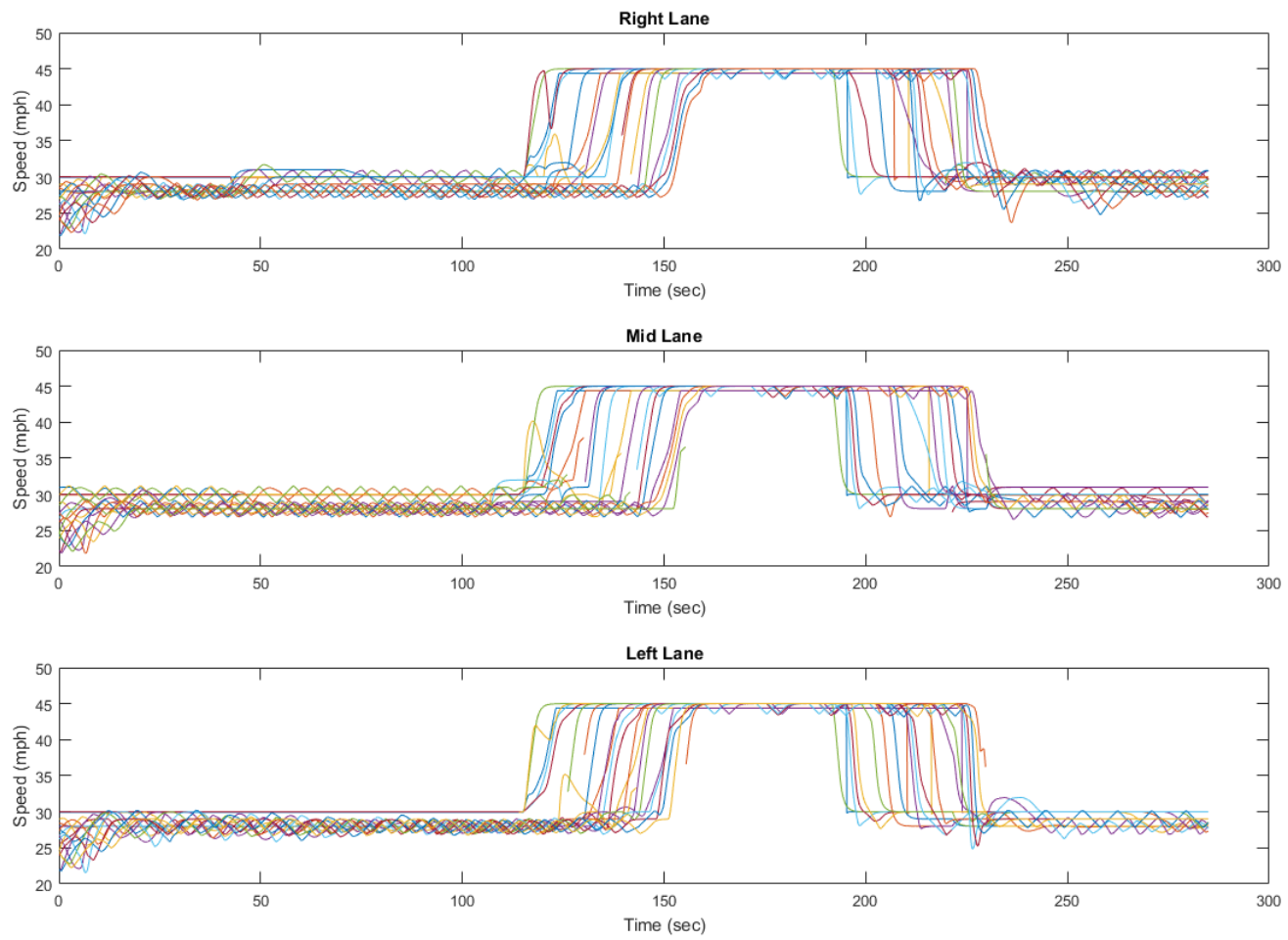

\section{Optimal Control Algorithm}
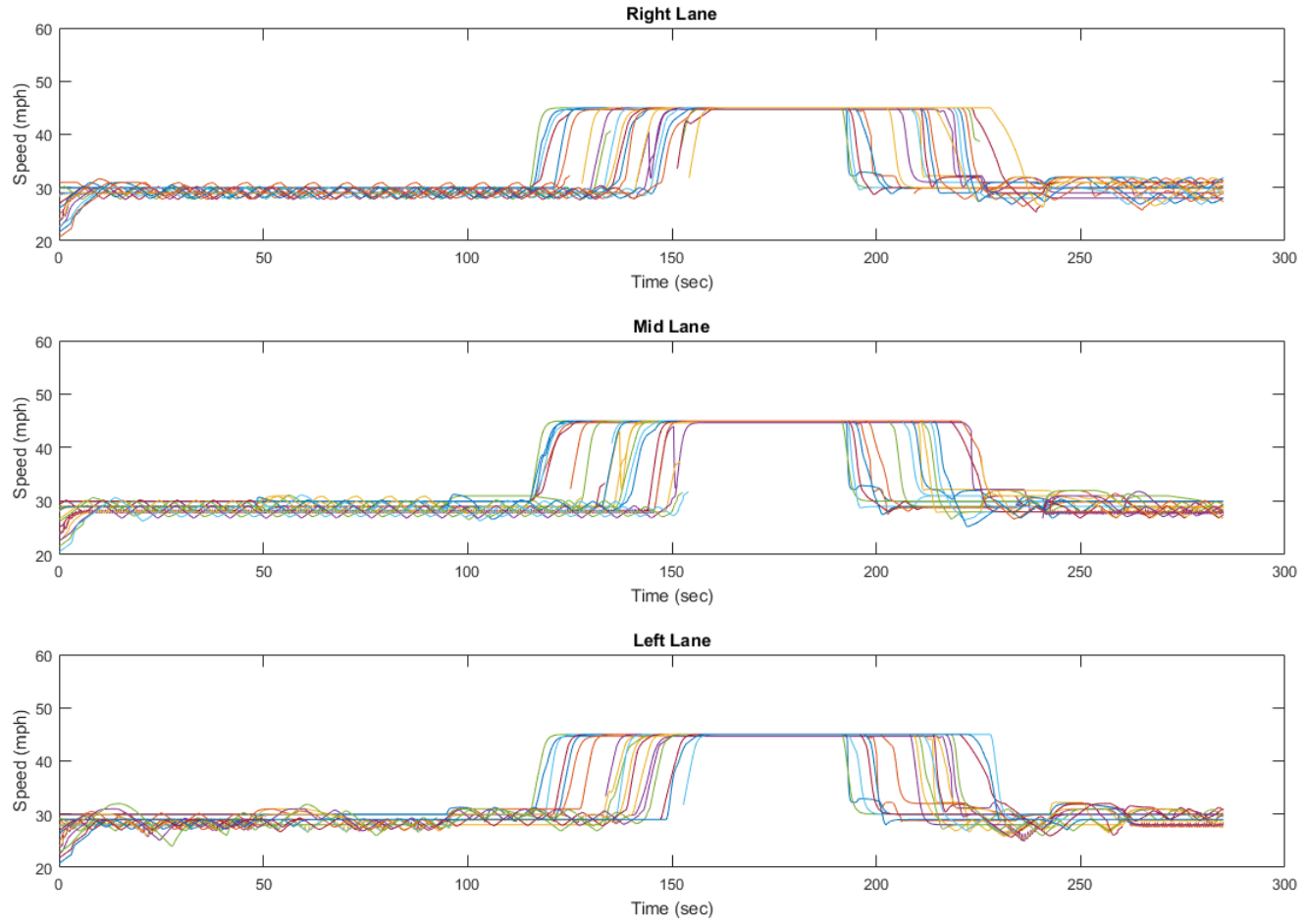


\section{E. $30 \%$ of $\mathrm{AV}$}

\section{ACC Algorithm}
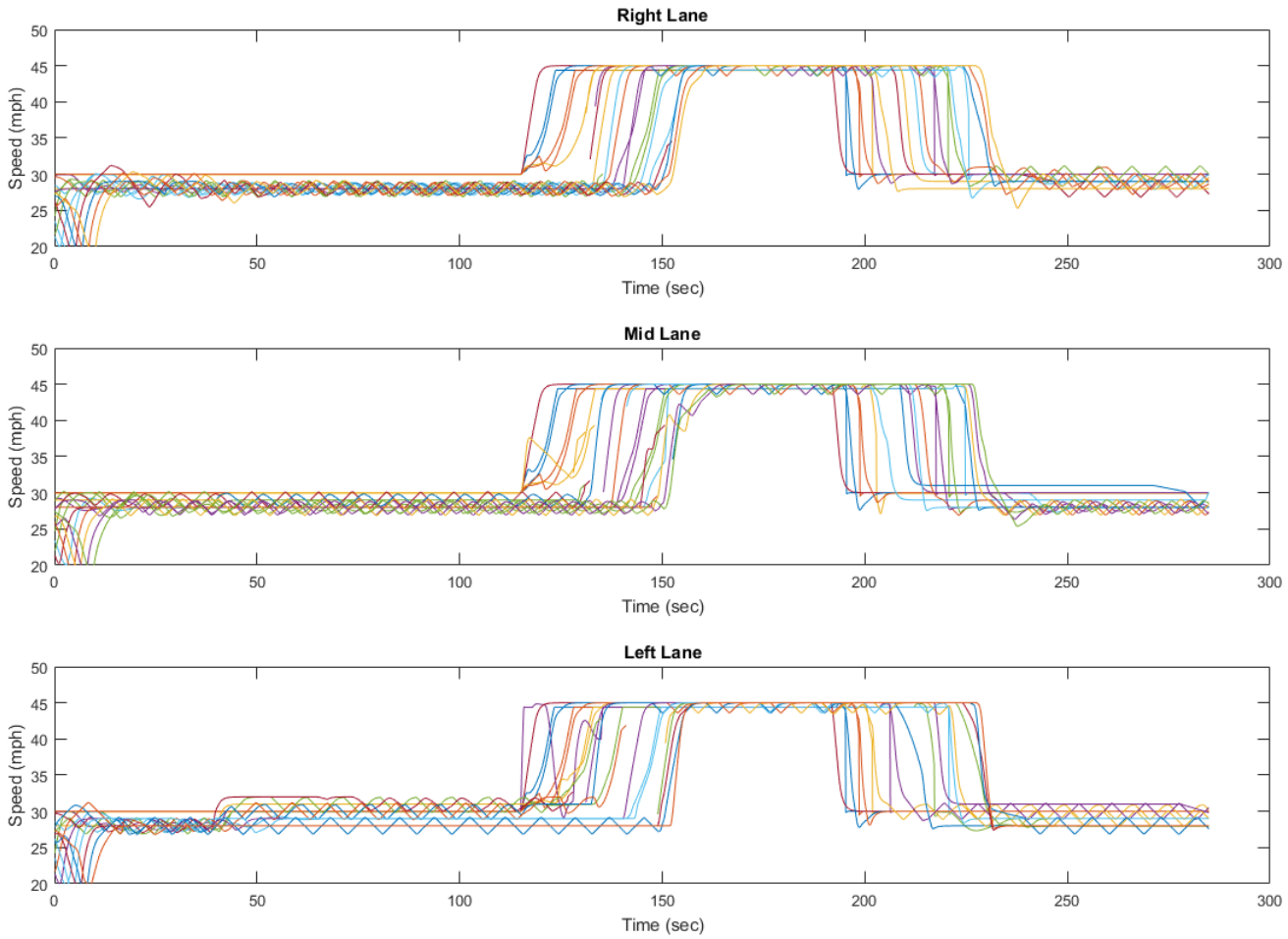

\section{Optimal Control Algorithm}
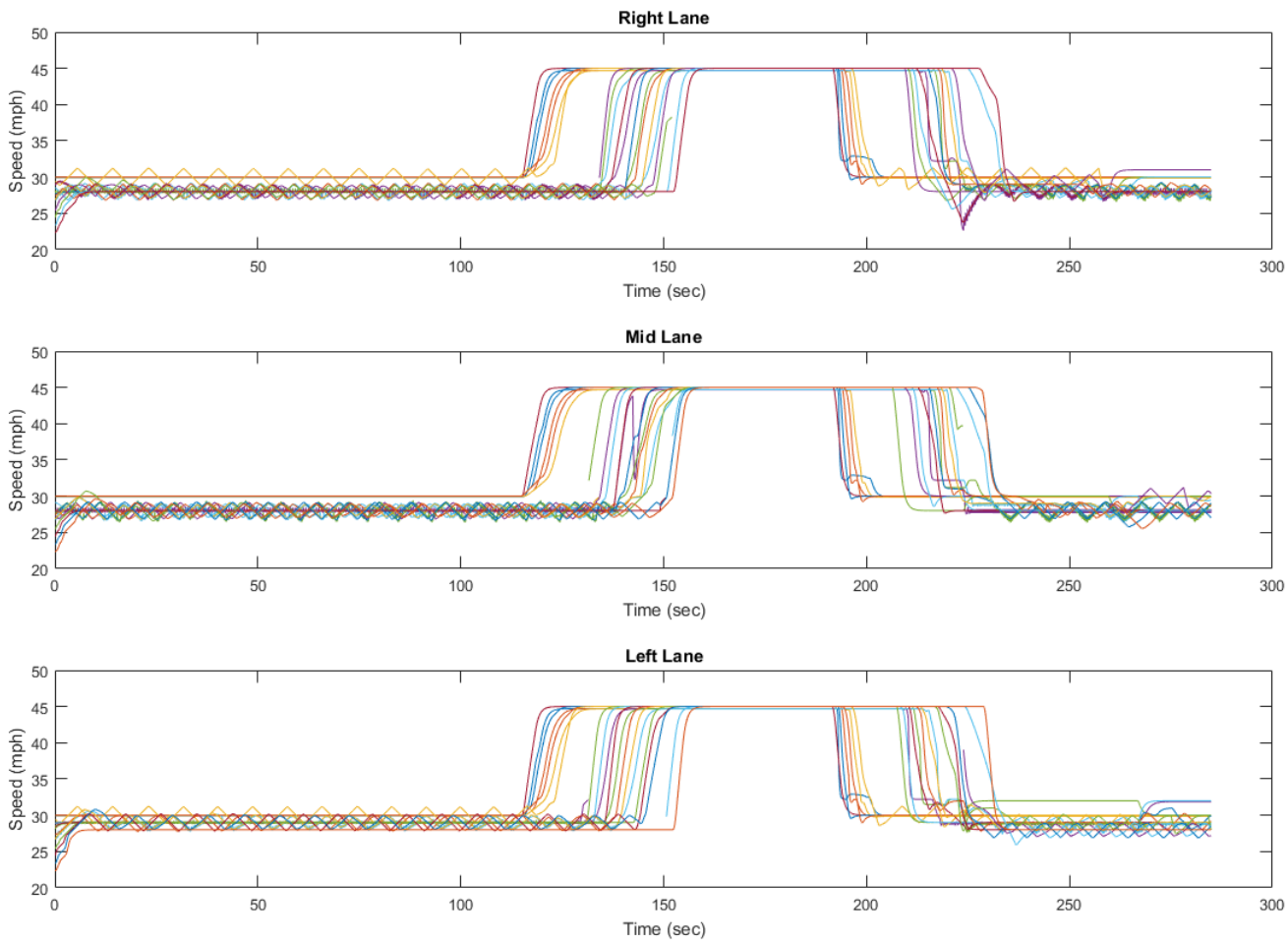


\section{F. $40 \%$ of $\mathrm{AV}$}

\section{ACC Algorithm}
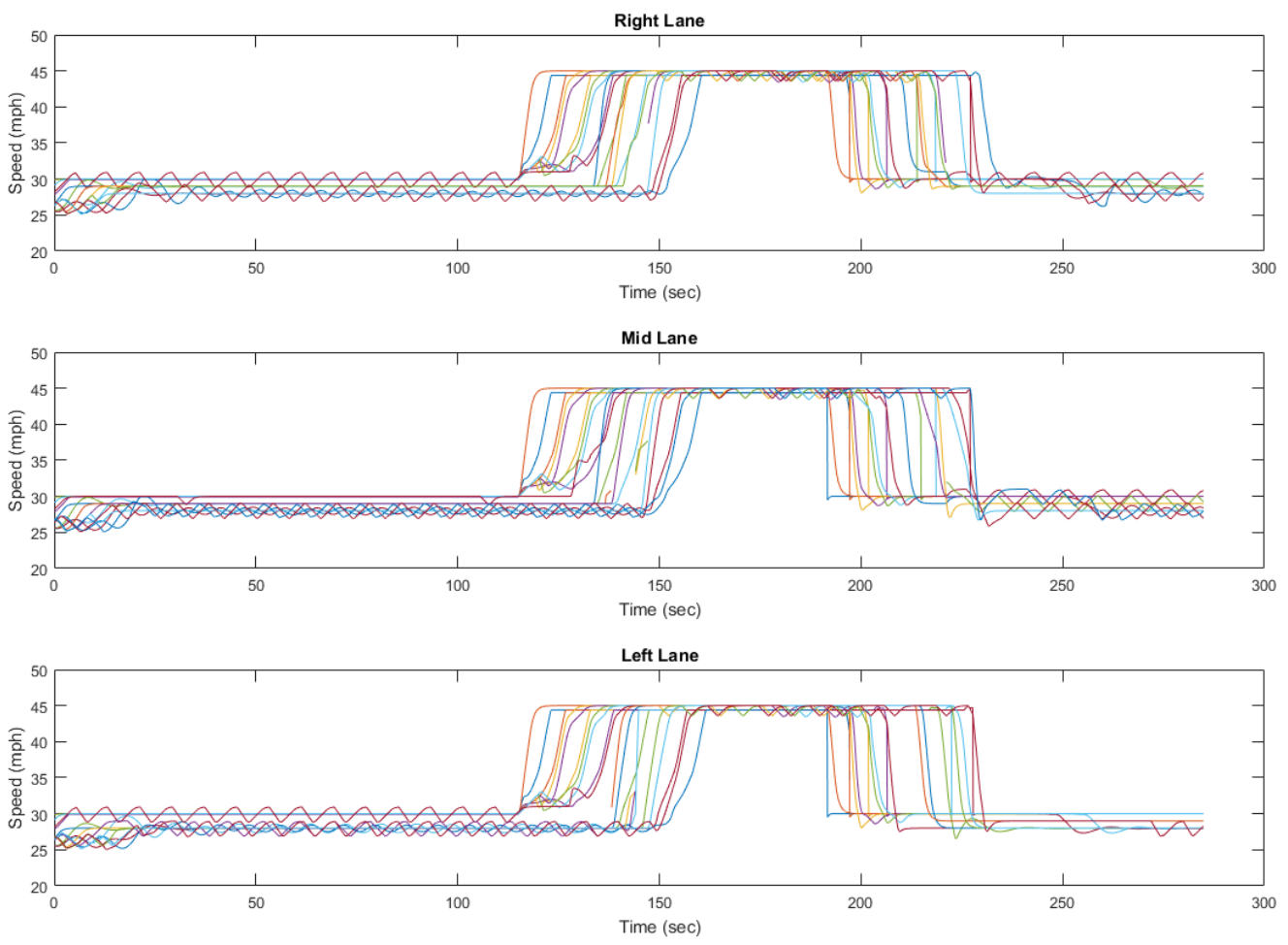

\section{Optimal Control Algorithm}
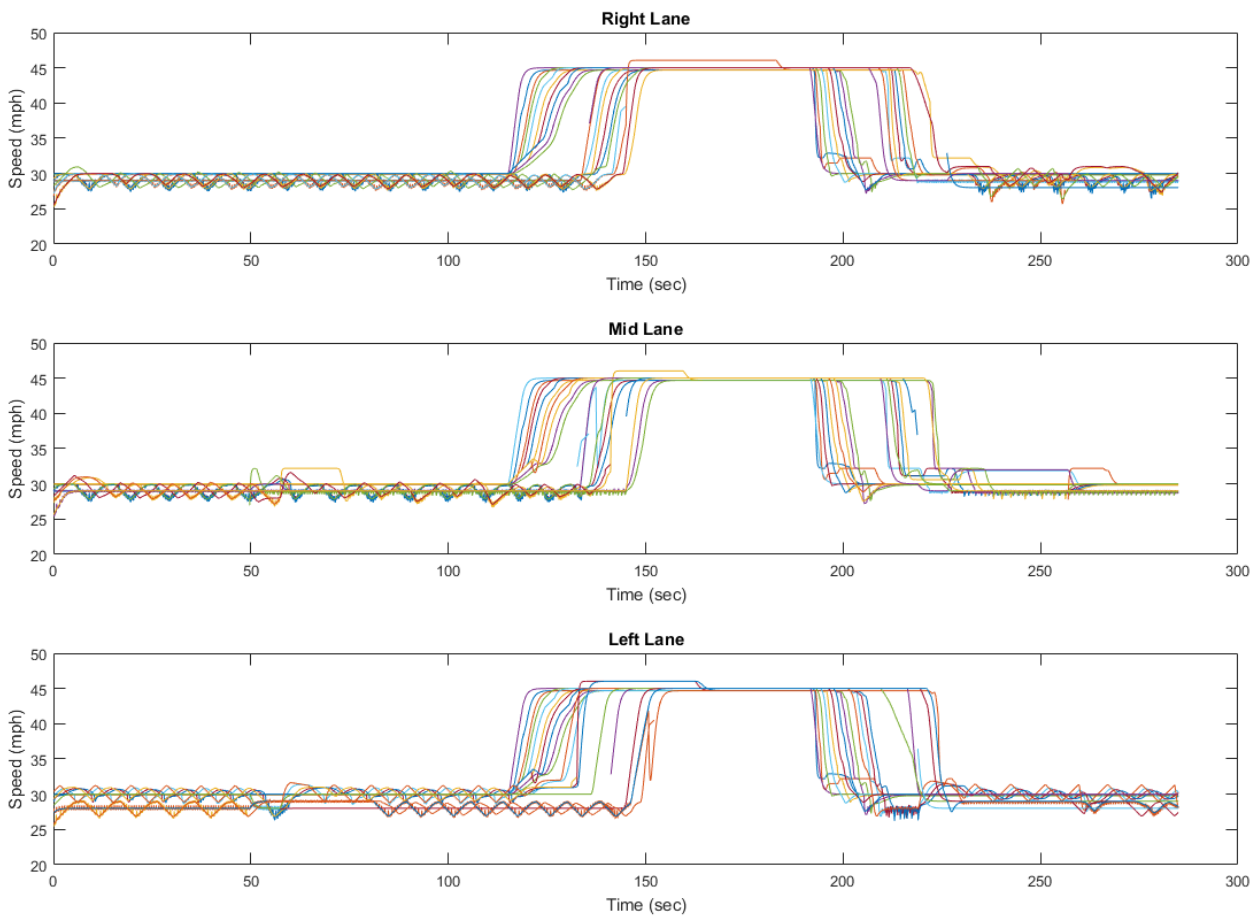


\section{G. $50 \%$ of $A V s$}

\section{ACC Algorithm}
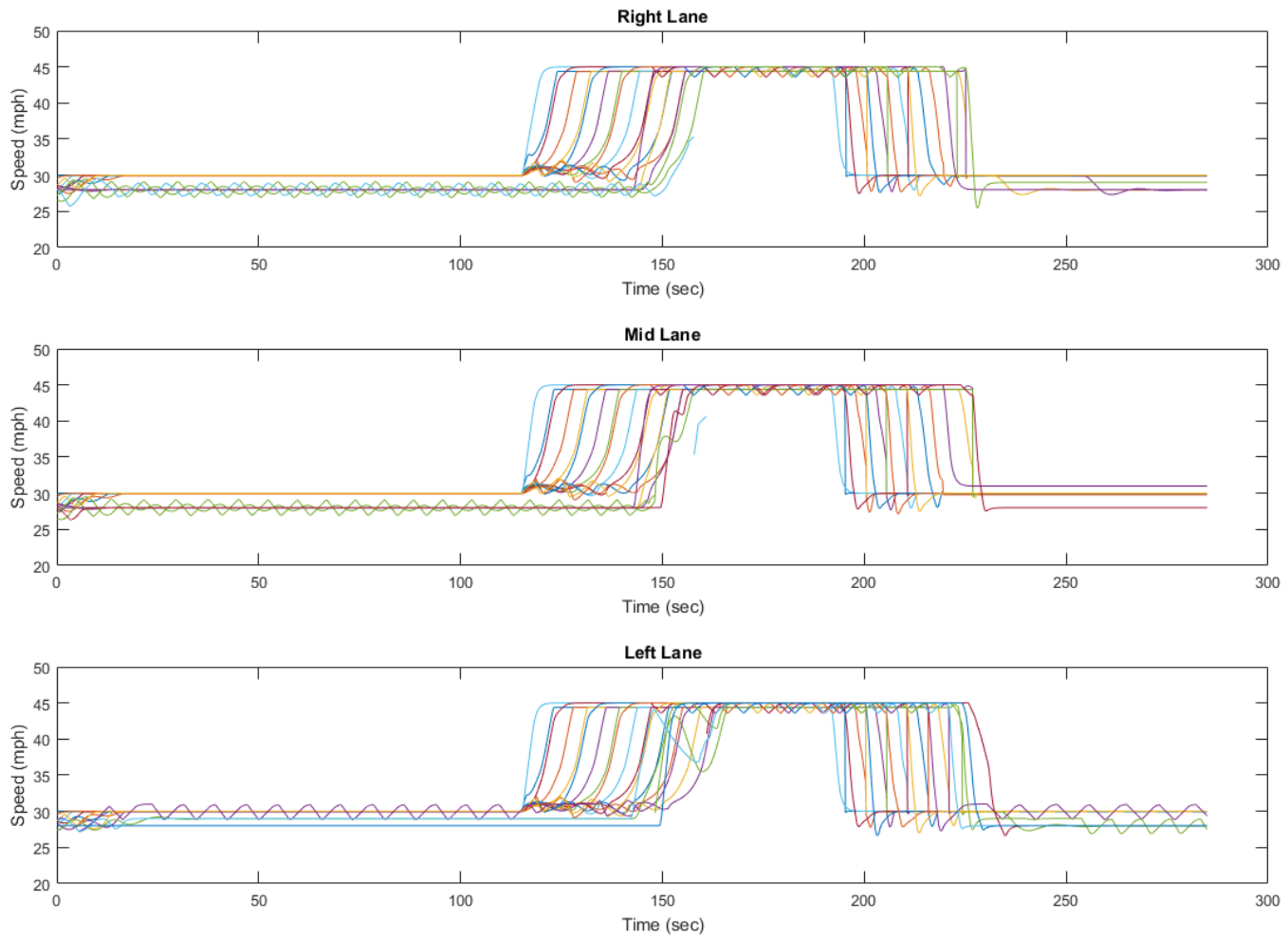

\section{Optimal Control Algorithm}
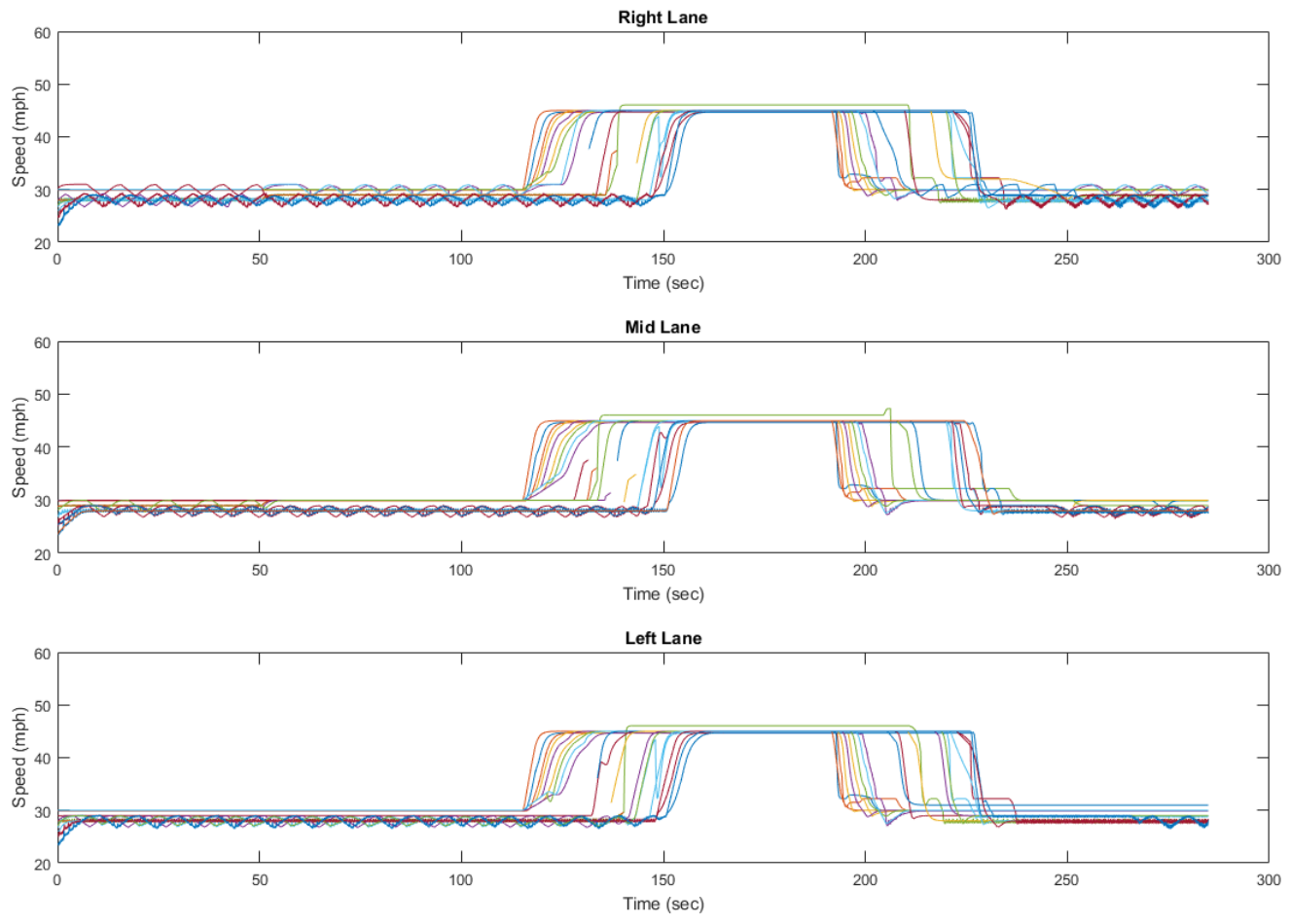


\section{H. $60 \%$ of AVs}

\section{ACC Algorithm}
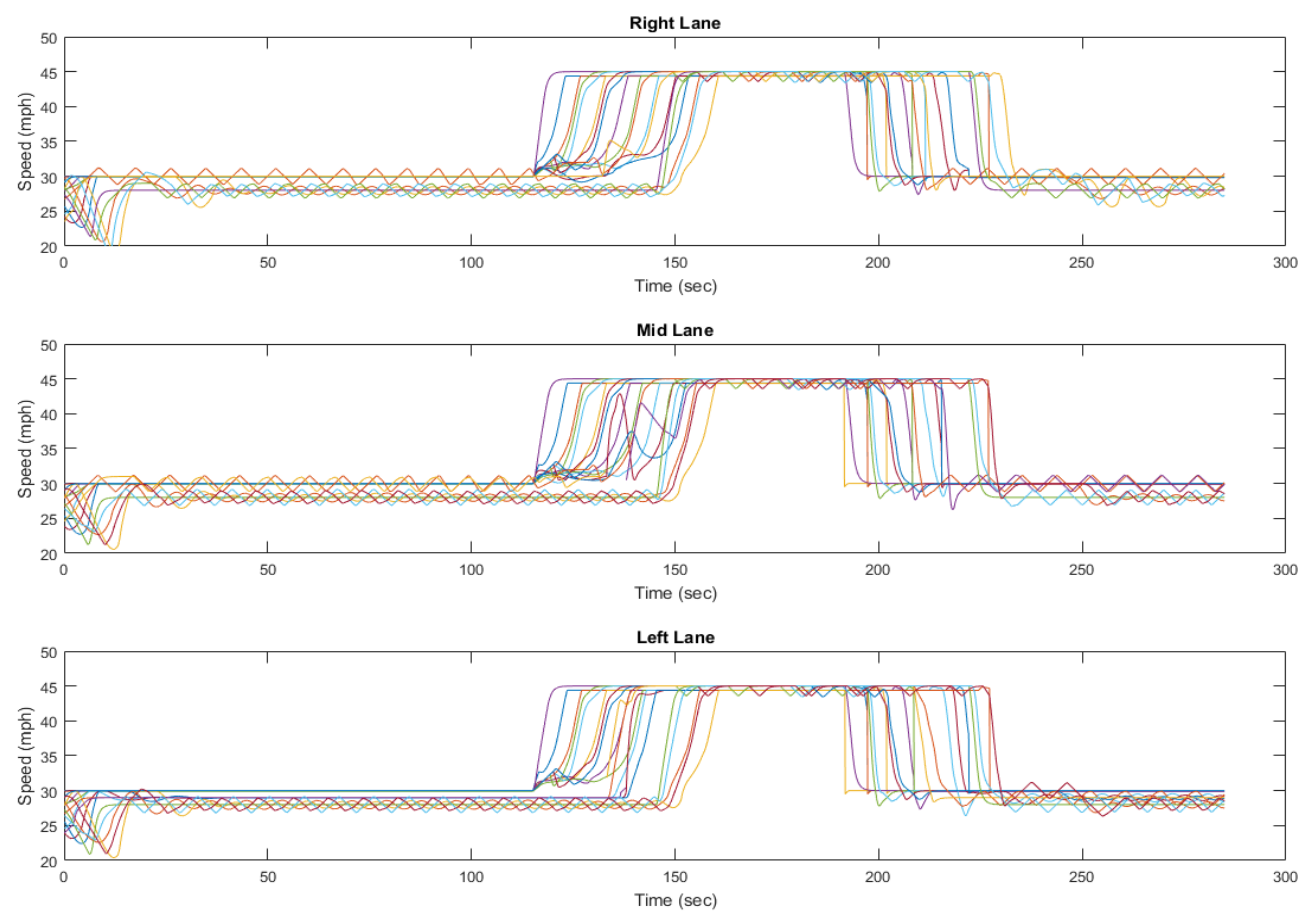

\section{Optimal Control Algorithm}
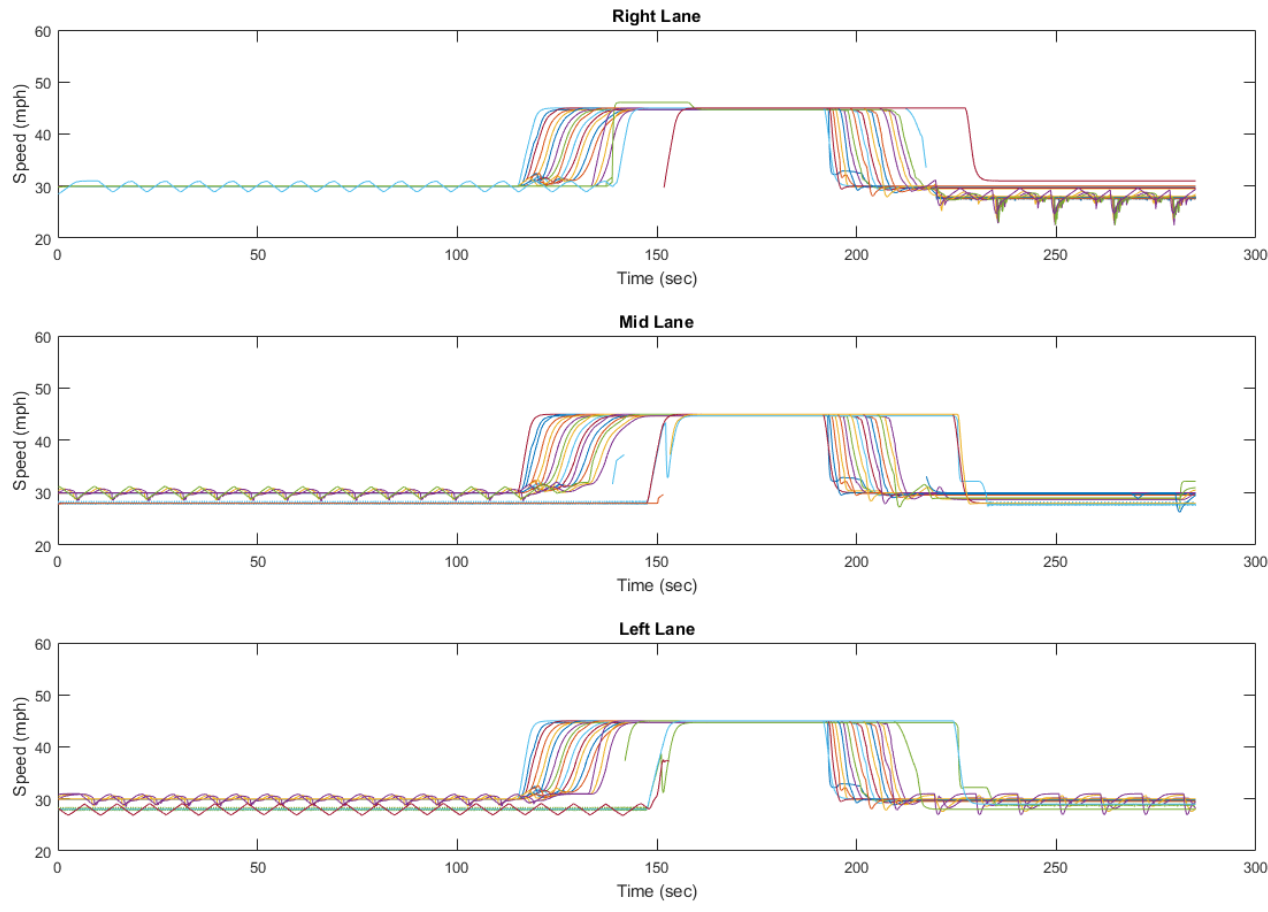


\section{I. $70 \%$ of $\mathrm{AV}$}

\section{ACC Algorithm}
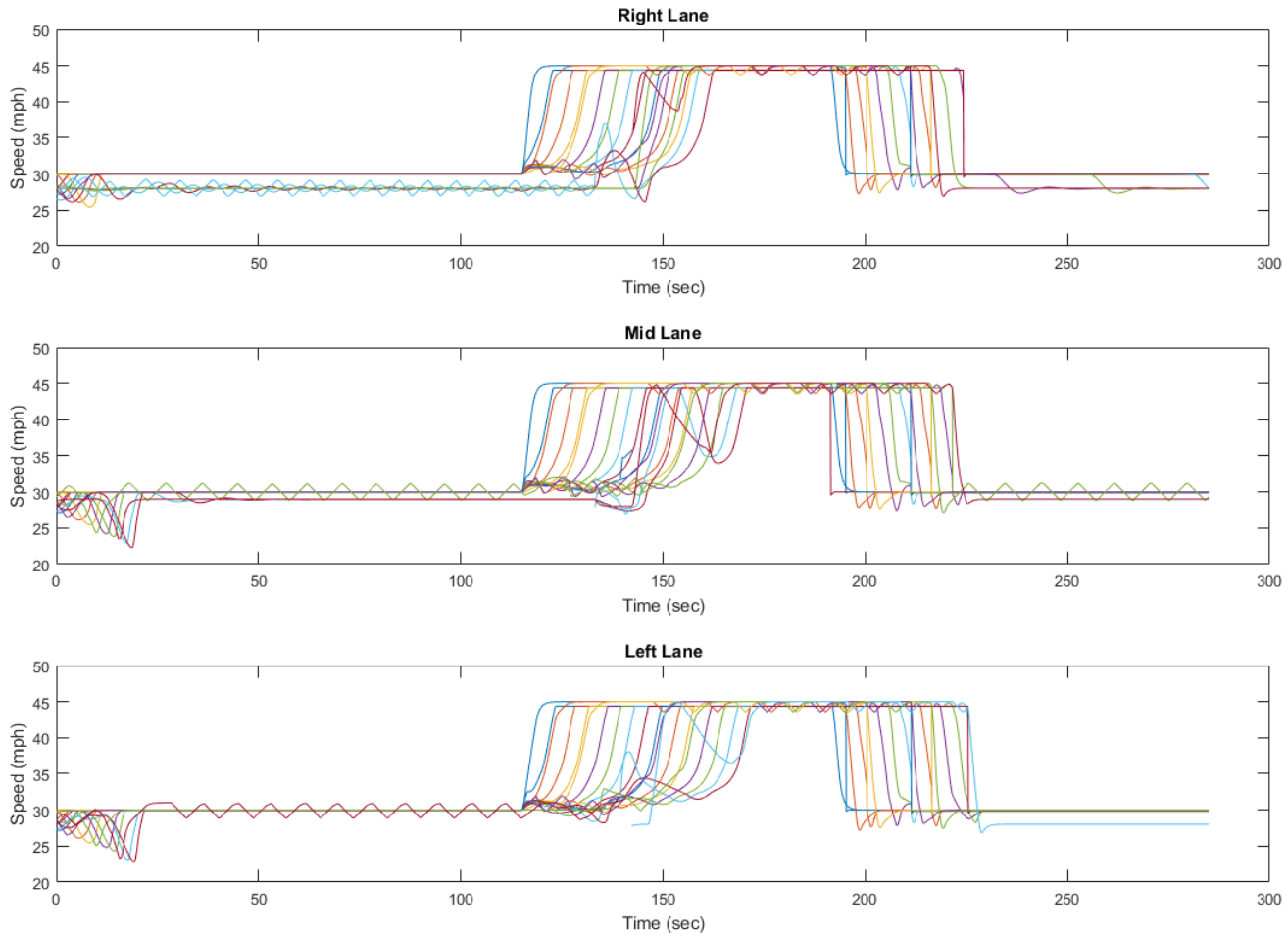

Optimal Control Algorithm
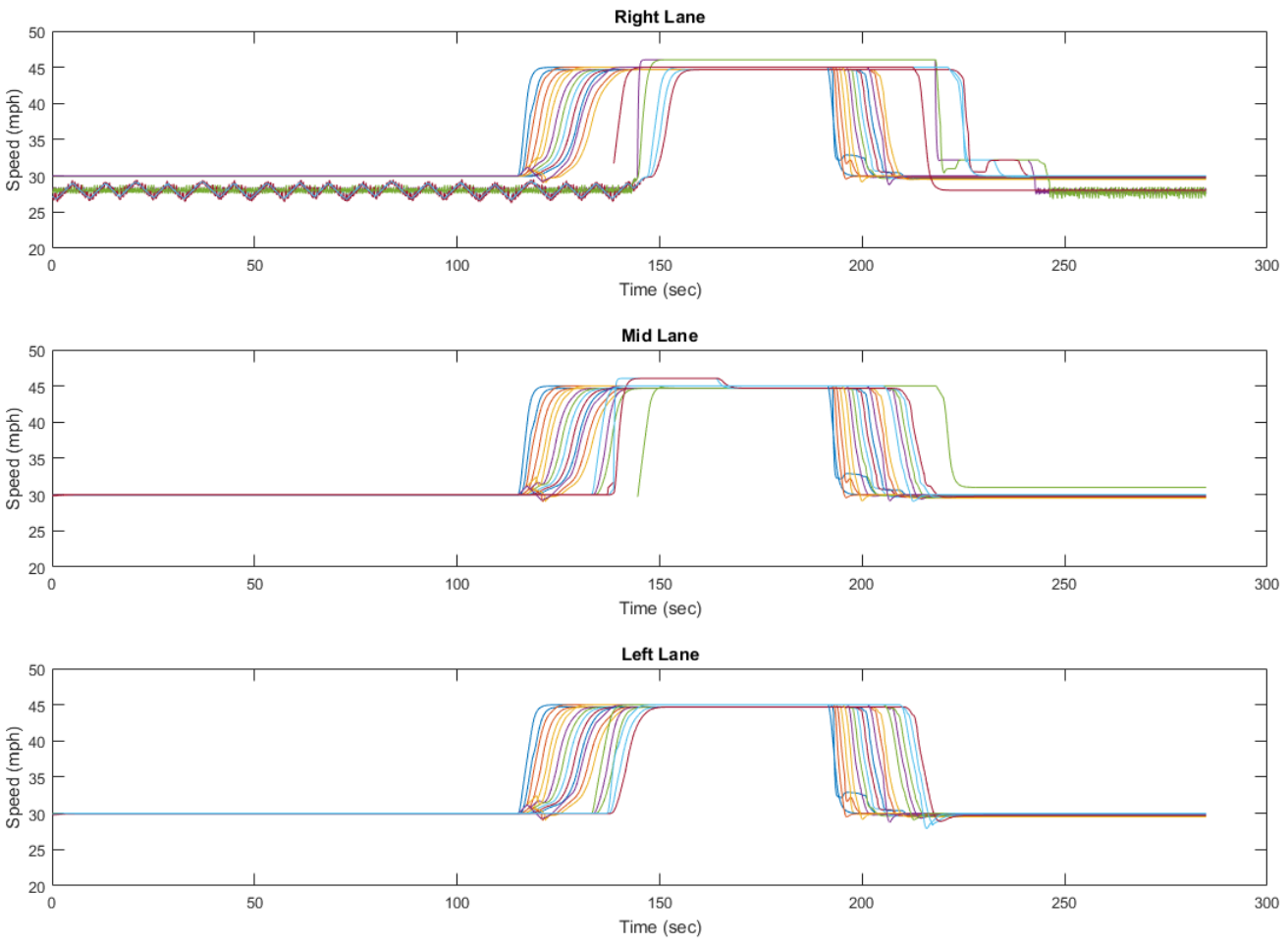


\section{J. $80 \%$ of $\mathrm{AV}$}

\section{ACC Algorithm}
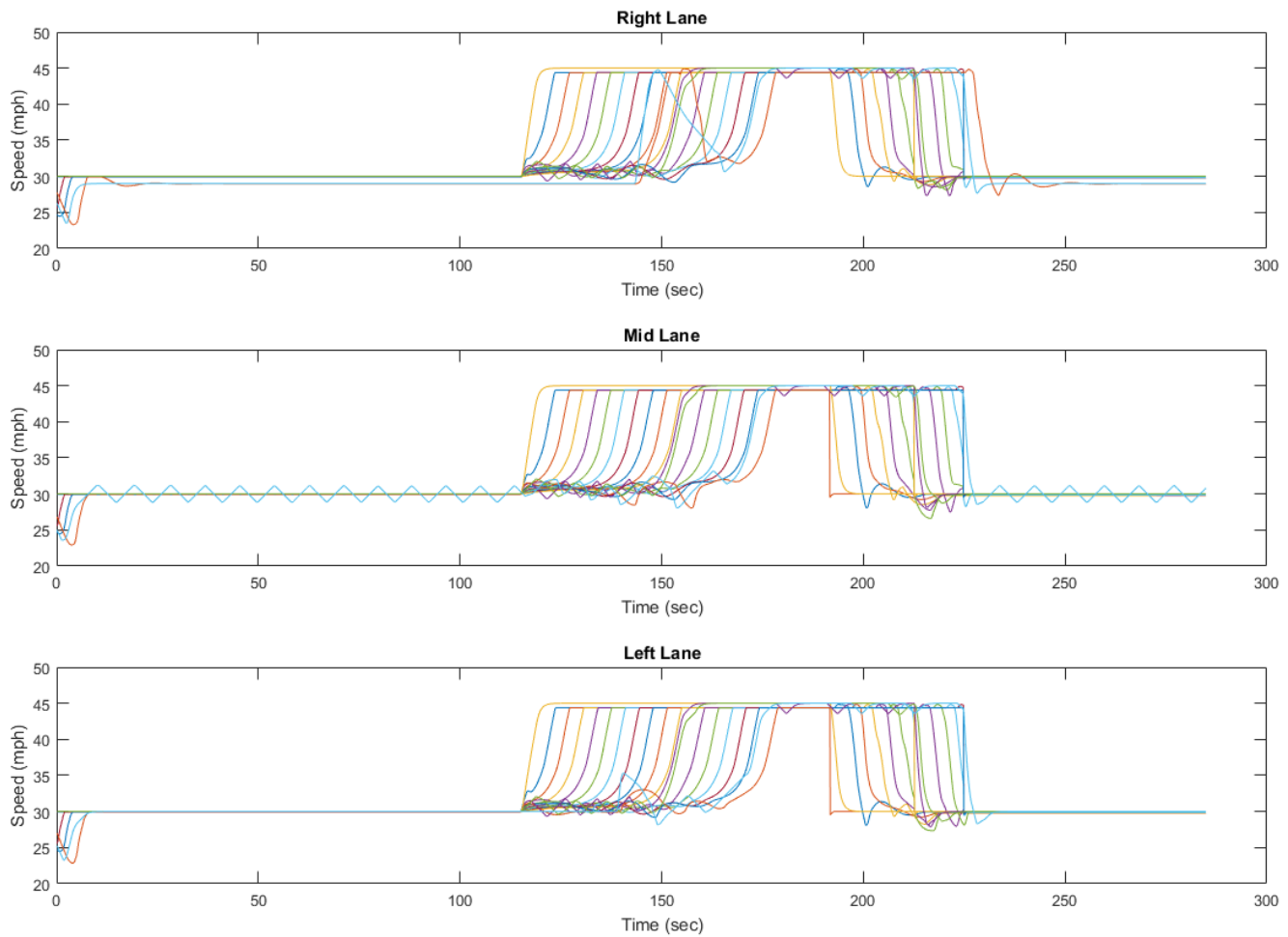

\section{Optimal Control Algorithm}
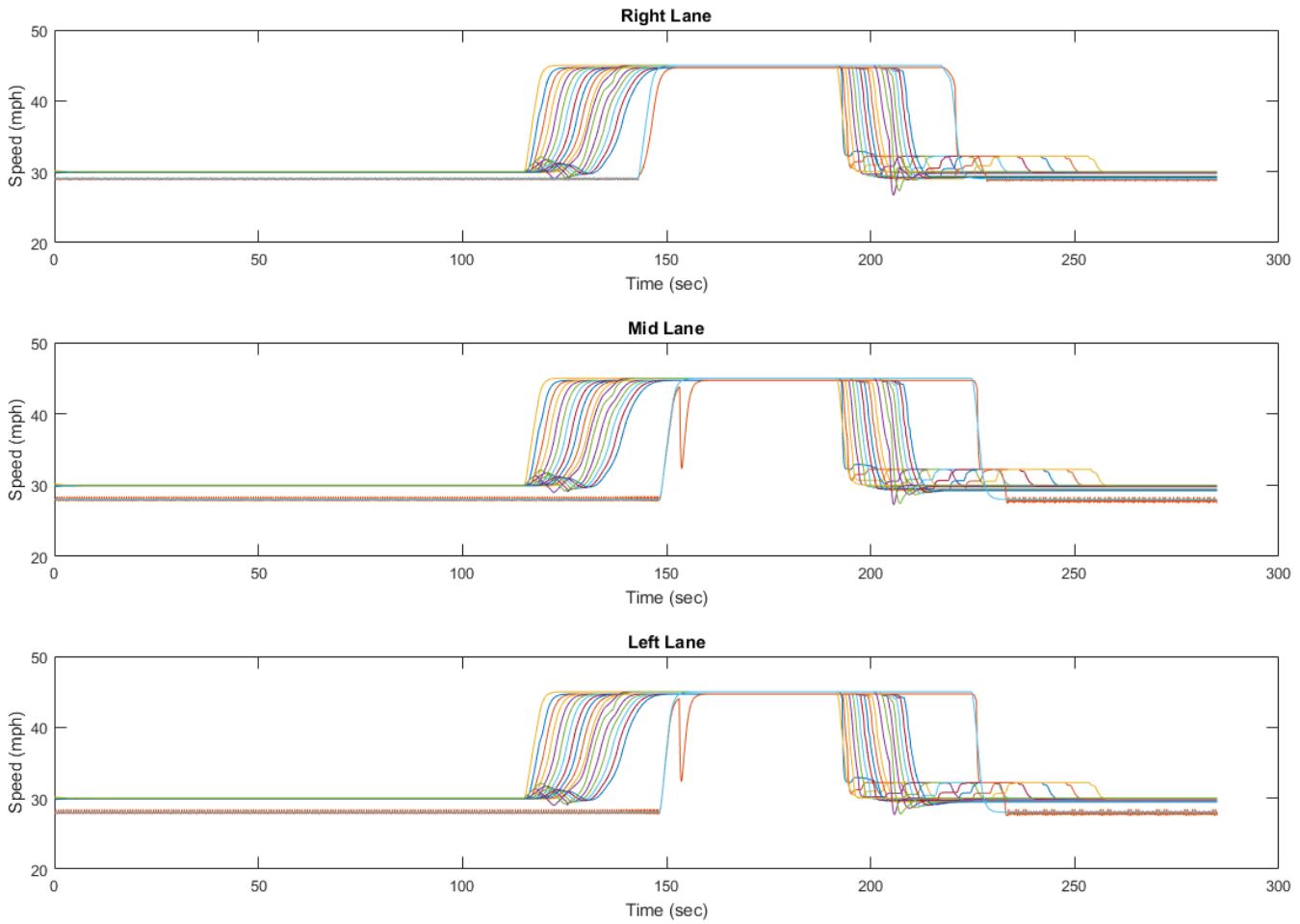


\section{K. $90 \%$ of $\mathrm{AV}$}

\section{ACC Algorithm}
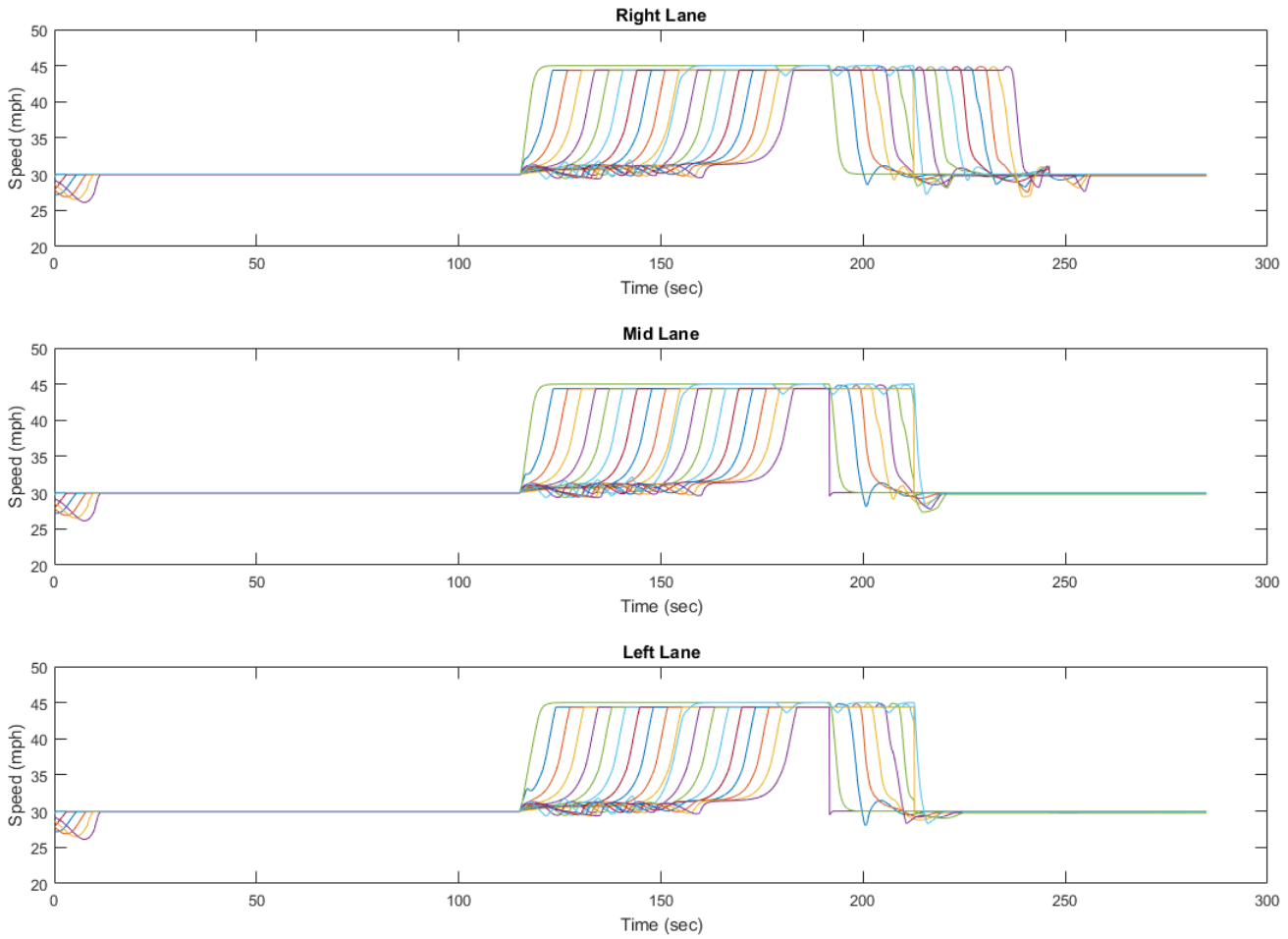

\section{Optimal Control Algorithm}
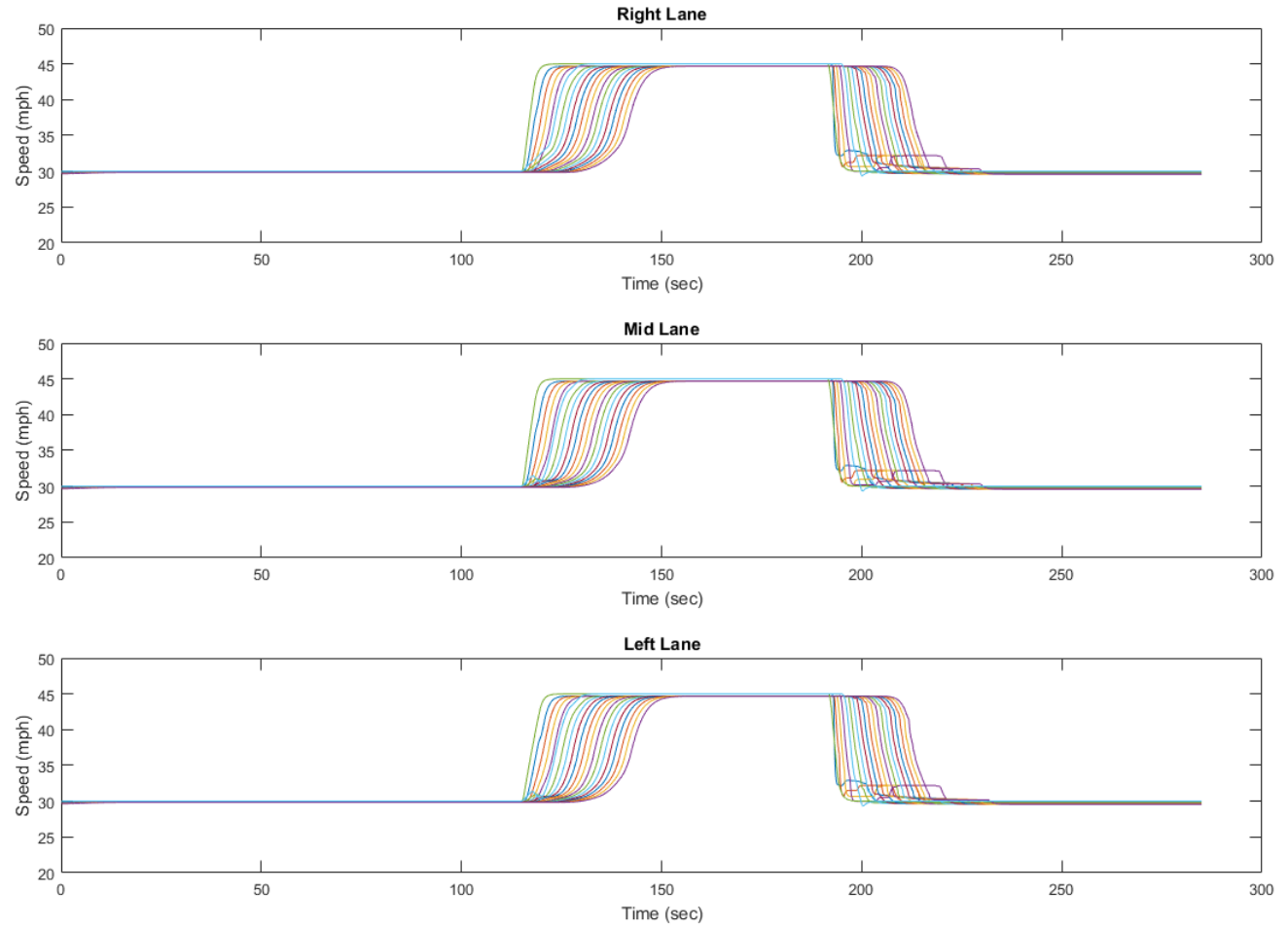


\section{L. $100 \%$ of AV}

\section{ACC Algorithm}
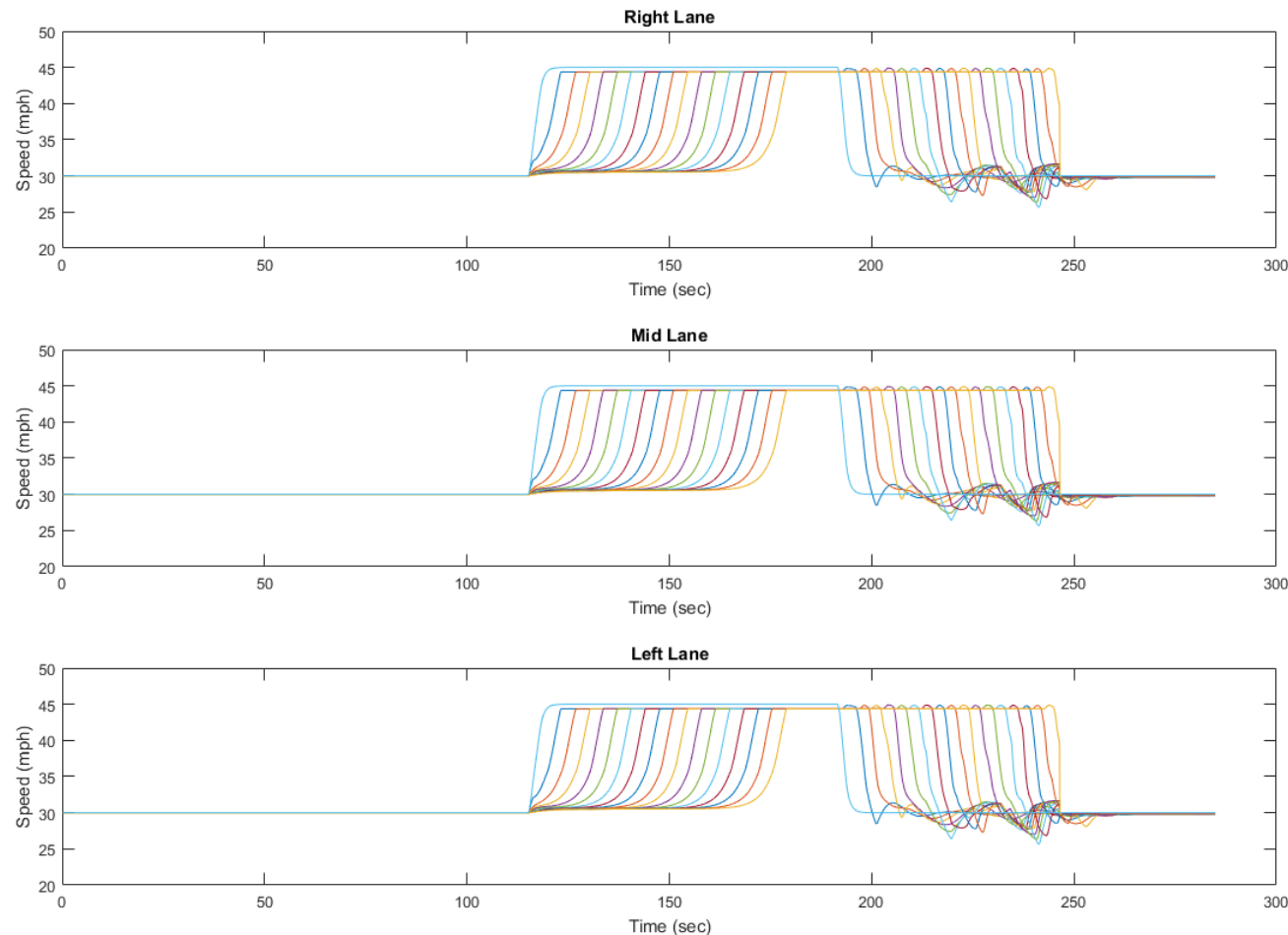

\section{Optimal Control Algorithm}
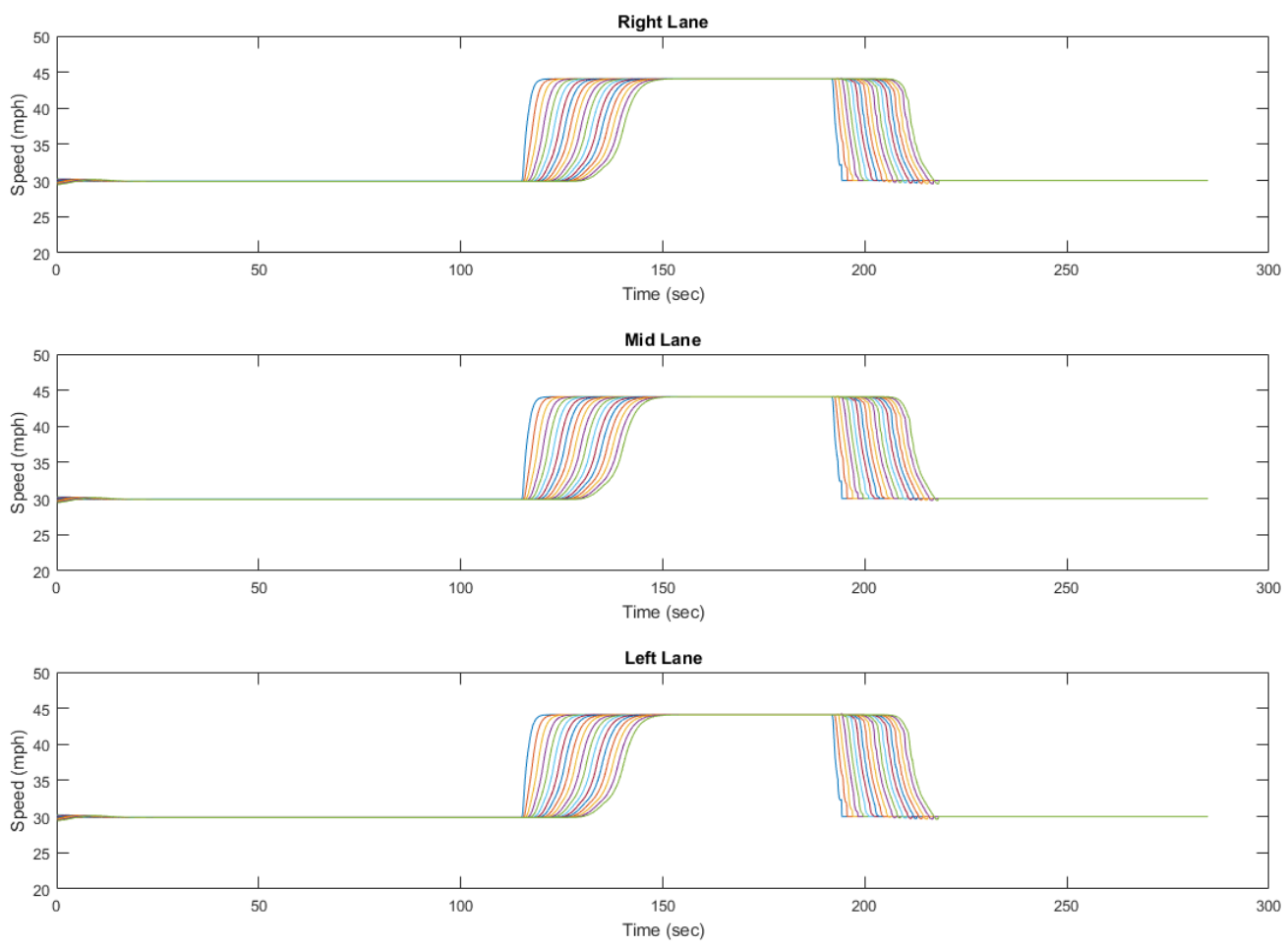